\title{
MÉTODOS DE OTIMIZAÇÃO APLICADOS À ANÁLISE DE ESTRUTURAS
}

\author{
Eng $^{0}$ Eduardo Rigo
}

Dissertação de Mestrado apresentada à Escola de Engenharia de São Carlos da Universidade de São Paulo, como parte dos requisitos para a obtenção do título de Mestre em Engenharia de Estruturas.

Orientador: Prof. Dr. Sérgio Persival Baroncini Proença

São Carlos 
\begin{tabular}{|l|} 
Chas. TESE-EESC, \\
Cutt. 4789 \\
Tombo $043 / 00$ \\
\hline
\end{tabular}

sis 1070398

Ficha catalográfica preparada pela Seção de Tratamento da Informação do Serviço de Biblioteca - EESC/USP

R572m

Rigo, Eduardo

Métodos de otimização aplicados à análise de estruturas / Eduardo Rigo. -- São Carlos, 1999.

Dissertação (Mestrado) -- Escola de Engenharia de São Carlos-Universidade de são Paulo, 1999.

Área: Engenharia de Estruturas.

Orientador: Prof. Dr. Sérgio Persival Baroncini Proença.

1. Métodos de otimização. 2. Método da energia. 3. Minimização com variáveis canalizadas. 4. Problemas de contato em estruturas. I. Título. 


\section{FOLHA DE APROVACÃO}

Candidato: Engenheiro EDUARDO RIGO

Dissertação defendida e aprovada em 22-10-1999 pela Comissão Julgadora:

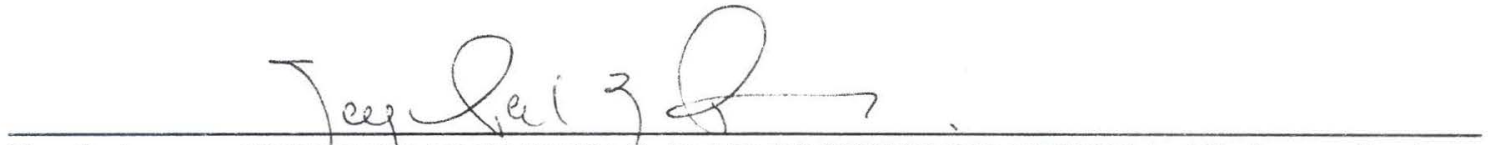

Prof. Assoc. SERGIO PERSIVAL BARONCINI PROENÇA (Orientador) (Escola de Engenharia de São Carlos - Universidade de São Paulo)

\section{Aloisio 2 Assan}

Prof. Assoc. ALOÍSIO ERNESTO ASSAN

(Universidade Estadual de Campinas - UNICAMP)

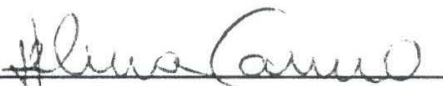

Profa. Dra. HELENA MARIA CUNHA DO CARMO ANTUNES

(Escola de Engenharia de São Carlos - Universidade de São Paulo)

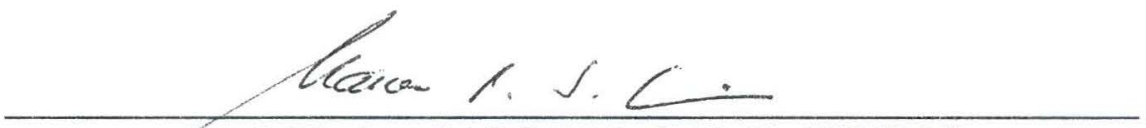

Prof. Titular CARLITO CALIL JUNIOR

Coordenador da Área de Engenharia de Estruturas

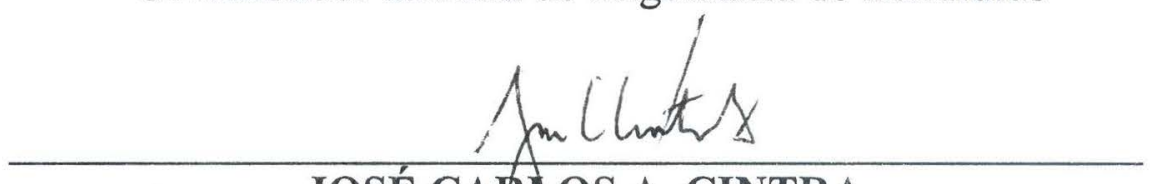

JOSÉ CARĹOS A. CINTRA

Presidente da Comissão ḍe Pós-Graduação da EESC 
Dedico essa pesquisa com todo meu amor e carinho à Elisangela, minha esposa, que sempre me apoiou e compreendeu o escasso tempo no decorrer do mestrado. 


\section{AGRADECIMENTOS}

Ao Prof. Dr. Sérgio P. B. Proença, reservo a minha maior gratidão, $\underline{\text { como }}$ profissional, pela responsabilidade, dedicação e competência demonstrada durante todo o processo de orientação do trabalho; como amigo, pelo respeito às minhas idéias e opiniões, pela generosidade e compreensão nos momentos difíceis, enfim, pela valiosa amizade que passamos a desfrutar.

Ao Prof. Dr. Marcos Nereu Arenales, do Instituto de Ciências Matemáticas de São Carlos, pela grande colaboração no estudo dos Métodos de Otimização.

Pela importante colaboração durante o exame de qualificação, pelas sugestões e incentivos, agradeço ao Prof. Dr. Libânio Miranda Pinheiro e $\operatorname{Prof}^{\mathrm{a}} \operatorname{Dr}^{\mathrm{a}}$ Helena M. C. Carmo Antunes, ambos do Departamento de Engenharia de Estruturas de São Carlos da Universidade de São Paulo.

Aos colegas, funcionários e professores do Departamento de Engenharia de Estruturas da Escola de Engenharia de São Carlos.

Aos amigos Faustino Sanches Jr. e Alexandre Sampaio Bota, pela grande amizade e apoio durante todos estes anos.

Ao Eng ${ }^{o}$ Sérgio Crespo pela amizade e colaboração.

Em especial aos meus pais, José Roberto Rigo e Sara Lorenzon Rigo, pela confiança e incentivo ao meu trabalho.

À Escola de Engenharia de São Carlos da Universidade de São Paulo, pela formação acadêmica.

À CAPES e CNPq, pelo apoio financeiro através da bolsa de estudo. 


\section{ÍNDICE}

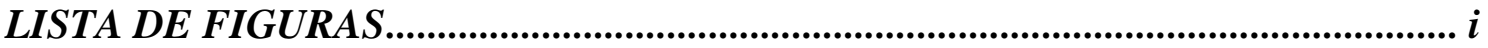

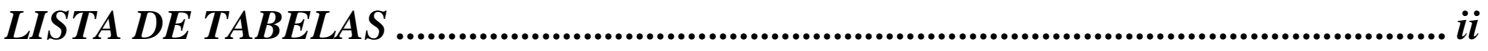

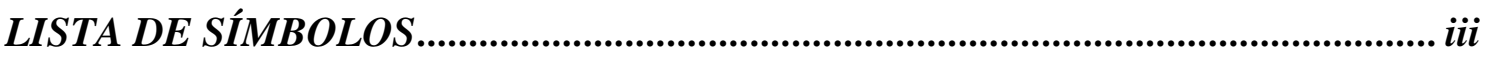

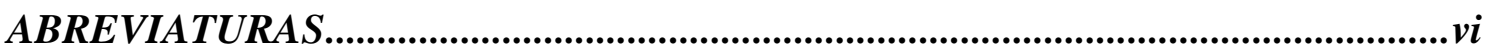

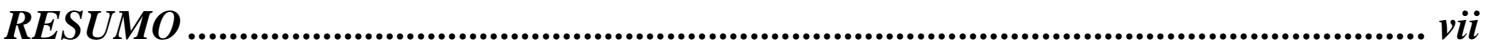

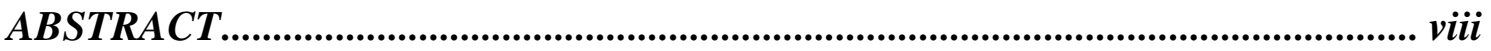

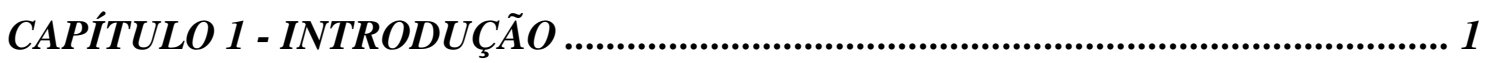

CAPÍTULO 2 - FORMULAÇÃO MATEMÁTICA ..................................................... 4

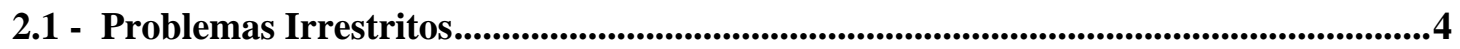

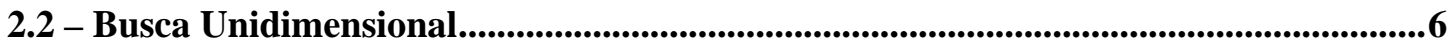

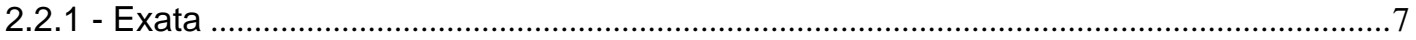

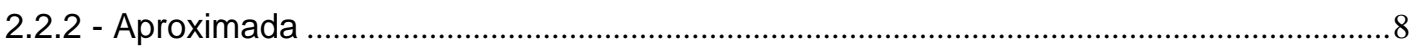

2.3 - Métodos de Minimização ............................................................................................10

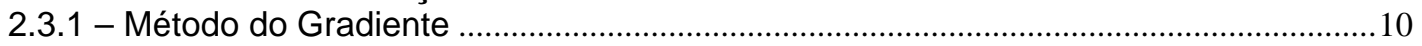

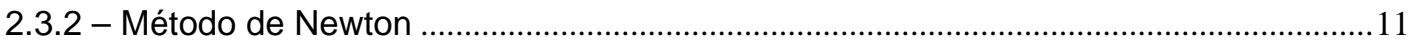

2.3.3 - Método de Quase-Newton .......................................................................................... 14

CAPÍTULO 3 - PROBLEMAS COM VARIÁVEIS CANALIZADAS ....................... 16

3.1 - Direção Factível.................................................................................................................................18

3.2 - Direção de Descida .....................................................................................................................20

3.3 - Método do Gradiente com variáveis canalizadas ..............................................................28

3.4 - Método de Gauss-Seidel................................................................................................37

3.5 - Método de Newton e Quase-Newton combinados com a estratégia dos Conjuntos

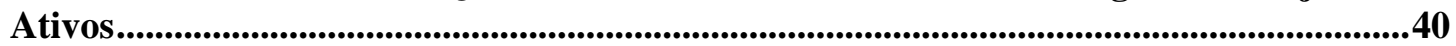

CAPÍTULO 4 - MODELAGEM DE ESTRUTURAS ............................................. 46

4.1 - Vigas ........................................................................................................................................................55

4.2 - Treliça Espacial_................................................................................................66

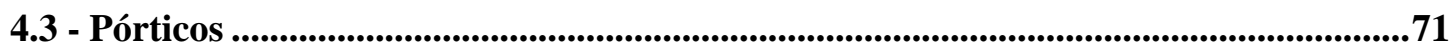

4.4 - Treliça Plana com não-linearidade geométrica...........................................................73 
CAPÍTULO 5 - EXEMPLOS DE APLICAÇÕES.

5.1 - Exemplo de Viga Contínua........................................................................................................80

5.2 - Exemplo de Pórtico Plano ...........................................................................................................82

5.3 - Exemplo de Treliça Espacial ..........................................................................................................84

5.4 - Exemplo de Viga Treliçada bi-apoiada .....................................................................................88

CAPÍTULO 6 - CONSIDERAÇÕES FINAIS E CONCLUSÕES........................... 91

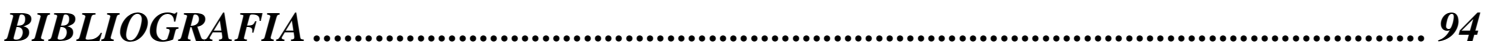

ANEXO 


\section{LISTA DE FIGURAS}

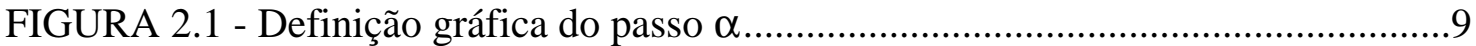

FIGURA 2.2 - Aproximação por uma função quadrática pelo Método de Newton .......12

FIGURA 3.1 - Ponto de mínimo restrito e irrestrito.........................................................17

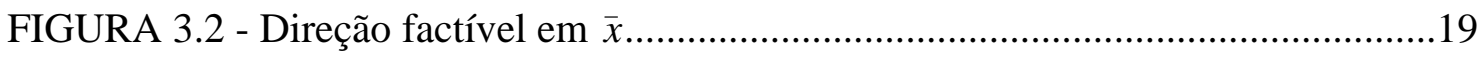

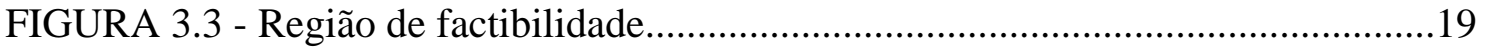

FIGURA 3.4 - Uma direção factível possível.............................................................20

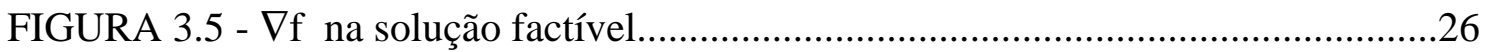

FIGURA 3.6 - Comprimentos e direções dos eixos de uma elipse...................................32

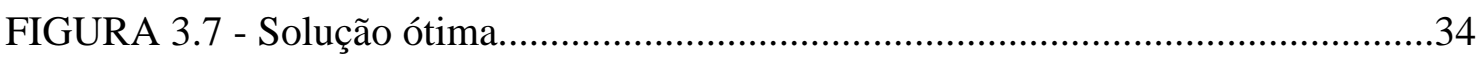

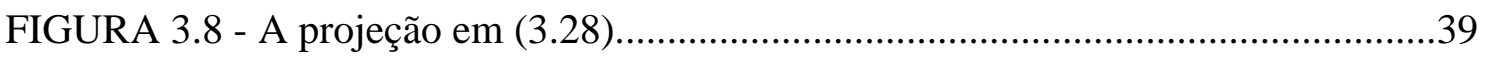

FIGURA 3.9 - Direção de Newton tridimensional..........................................................40

FIGURA 3.10 - Minimização numa aresta..................................................................

FIGURA 4.1 - Representação esquemática dos deslocamentos no plano médio.............55

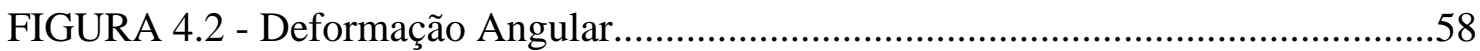

FIGURA 4.3 - Graus de liberdade ou coordenadas adotadas.........................................61

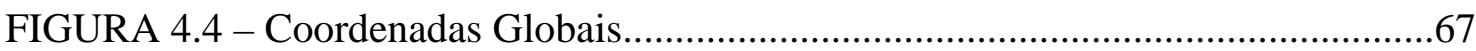

FIGURA 4.5 - Coordenadas Locais segundo o Sistema Global.....................................68

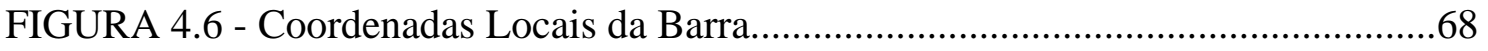

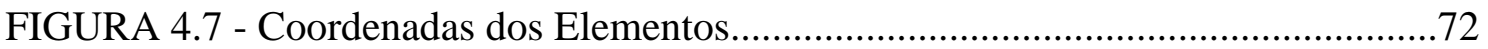

FIGURA 4.8 - Elemento finito de treliça plana............................................................ 74

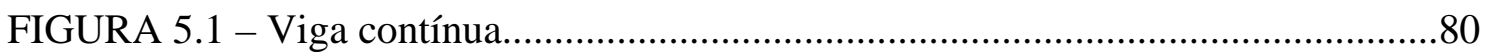

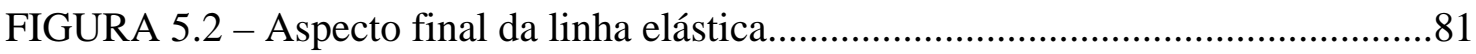

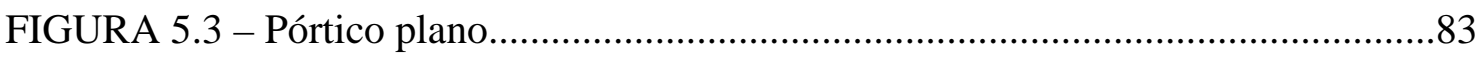

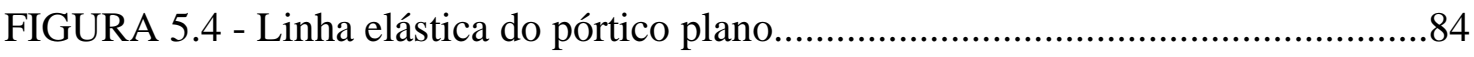

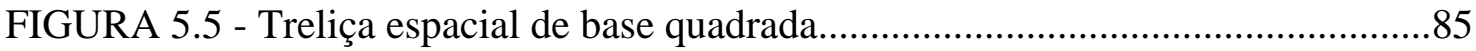

FIGURA 5.6 - Configuração final da elástica da treliça espacial..................................87

FIGURA 5.7 - Viga simétrica bi-apoiada - Problema de contato..................................89

FIGURA 5.8 - Linha elástica da viga simétrica bi-apoiada.........................................90 


\section{LISTA DE TABELAS}

TABELA 5.1 - Comparação dos métodos para viga contínua. .81

TABELA 5.2 - Convergência do método do Gradiente....................................................82

TABELA 5.3 - Comparação dos métodos para pórtico plano...........................................83

TABELA 5.4 - Treliça espacial sem o problema de contato unilateral (minimização irrestrita).

TABELA 5.5 - Treliça espacial com problema de contato unilateral (minimização restrita). .86

TABELA 5.6 - Deslocamento vertical segundo o carregamento. .90 


\section{LISTA DE SÍMBOLOS}

$\dot{u}=$ derivada do deslocamento em relação ao tempo

$|\hat{S}|=$ matriz representativa do tensor de tensão de Piola-Kirchhoff de $2^{\text {a }}$ espécie

$\dot{t}_{0}=$ taxa de força por unidade de área na configuração inicial

$\dot{r}=$ taxa de resíduo

$\dot{\varepsilon}=$ taxa do tensor de deformação de Green na configuração atual

$\delta \dot{\varepsilon}=$ taxa do tensor de deformação virtual de Green na configuração inicial

$\dot{S}=$ taxa do tensor de tensão de Piola-Kirchhoff de $2^{\text {a }}$ espécie na configuração inicial

$\dot{q}=$ taxa do vetor de deslocamentos nodais

$\delta \varepsilon=$ tensor de deformação virtual de Green na configuração inicial

$\delta q=$ vetor de deslocamentos virtuais nodais

$\lambda=$ auto-valor

$v=$ coeficiente de Poisson

$\gamma=$ deformação transversal

$\Psi=$ energia potencial de carga

$\pi=$ energia potencial total

$\varphi=$ função unidimensional

$\Phi=$ matriz de direções da face

$\phi=$ matriz de funções de forma

$\beta=$ matriz de incidência cinemática

$\Lambda=$ matriz diagonal dos auto-valores

$\Omega=$ região no $\mathrm{R}^{\mathrm{n}}$

$\theta=$ rotação do nó (giro)

$\Gamma=$ superfície do elemento

$\alpha=$ tamanho do passo

$\varepsilon=$ tensor de deformação de Green

$\sigma=$ tensor de tensão de Cauchy

$\tau=$ tensor de tensão de Kirchhoff-Treftz

$\mathrm{T}=$ trabalho da carga

$\eta=$ valor constante 
$\delta=$ vetor deslocamento

$\nabla^{2} \mathrm{f}=$ segunda derivada de $f$, ou seja, sua matriz hessiana

$\Psi_{\mathrm{a}}=$ energia potencial de carga aproximada

$\pi_{\mathrm{a}}=$ energia potencial total aproximada

$\nabla \mathrm{f}=$ primeira derivada de $f$, ou seja, seu vetor gradiente

$\alpha_{\mathrm{k}}=$ tamanho do passo na iteração $k$

$\mathrm{A}=$ área da seção transversal

$a, b=$ vetores de restrição de variáveis

$\mathrm{B}, \mathrm{B}_{0}$ e $\mathrm{B}_{\mathrm{L}}=$ matrizes auxiliares

$\mathrm{C}=$ valor escalar

$\mathrm{C}_{\mathrm{x}}, \mathrm{C}_{\mathrm{y}}$ e $\mathrm{C}_{\mathrm{z}}=$ cossenos diretores na direção $x, y$, e $z$ respectivamente

$\mathrm{D}=$ matriz constitutiva do material

$\mathrm{d}^{\mathrm{k}}=$ direção de descida na iteração $k$

$\mathrm{E}=$ módulo de elasticidade longitudinal

$\mathrm{f}$ = função quadrática

$\mathrm{F}=$ vetor de forças nodais

$\mathrm{g}=$ força da gravidade

$\mathrm{G}=$ módulo de elasticidade transversal

$\mathrm{H}=$ matrizes hessianas

$\mathrm{I}=$ momento de inércia

$\mathrm{K}_{\sigma}=$ matriz de rigidez geométrica

$\mathrm{K}, \mathrm{R}=$ matrizes de rigidez

$\mathrm{K}_{0}=$ matriz de rigidez elástica linear

$\mathrm{k}_{1}, \mathrm{k}_{2}, \ldots \mathrm{k}_{\mathrm{n}}=$ valores reais constantes

$\mathrm{K}_{\mathrm{L}}=$ matriz de rigidez de correção de coordenadas

$\mathrm{K}_{\mathrm{T}}=$ matriz de rigidez tangente

$\mathrm{L}=$ comprimento da barra

$\mathrm{M}=$ momento fletor

$\mathrm{p}=$ força de superfície

$\mathrm{P}=$ vetor de cargas nodais

$\mathrm{P}_{\mathrm{e}}=$ vetor de cargas nodais do elemento

$\mathrm{Q}=$ matriz de auto-vetor

$q=$ vetor de deslocamentos nodais 
$\mathrm{q}(\mathrm{x})$ = função quadrática de aproximação

$\mathrm{r}=$ raio de curvatura

$\mathrm{r}_{\mathrm{e}}=$ matriz de rigidez do elemento

$\mathrm{S}=$ vetor auxiliar

$\mathrm{U}=$ energia de deformação

$\mathrm{u}=$ vetor deslocamento

$\mathrm{u}_{1}, \mathrm{u}_{2}, \ldots \mathrm{u}_{\mathrm{n}}=$ componentes de deslocamento

$\mathrm{U}_{\mathrm{a}}=$ energia de deformação aproximada

$\mathrm{v}=$ vetor de deslocamento vertical

$\mathrm{V}=$ volume

$\mathrm{W}=$ valor da relaxação

$\mathrm{x}=$ vetor solução

$\mathrm{x}_{\mathrm{i}}=$ componente $\mathrm{i}$ do vetor $\mathrm{x}$

$\mathrm{y}=$ coordenada

$\mathrm{Y}_{\mathrm{k}}=$ inversa da matriz hessiana na iteração $k$

$\mathrm{z}=$ coordenada 


\section{ABREVIATURAS}

cte $=$ constante

DFP $=$ Davidon-Fletcher-Powell

$\min =$ minimização

PTV = Princípio dos Trabalhos Virtuais

tol $=$ tolerância 


\section{RESUMO}

RIGO, E. (1999). Métodos de Otimização aplicados à Análise de Estruturas. São Carlos. 105p. Dissertação (Mestrado). Escola de Engenharia de São Carlos, Universidade de São Paulo.

O Método dos Elementos Finitos quando aplicado à análise de estruturas, em sua forma usual, conduz a sistemas de equações que, no caso não-linear, exigem algoritmos iterativos que realizam, em essência, uma linearização a cada passo de carga. Por outro lado, o Método da Energia formula o problema de análise estrutural na forma de uma minimização, podendo apresentar restrições sobre a função deslocamento, por exemplo. Nesse caso, os algoritmos de programação matemática proporcionam a maneira mais consistente para a obtenção da solução.

O presente trabalho de mestrado trata, essencialmente, da aplicação das técnicas de otimização como ferramenta para a análise do comportamento não-linear de estruturas, que pode ser decorrente de condições de vinculação. Os problemas estruturais são formulados via Método da Energia, que resulta na minimização de funções quadráticas sujeitas a um conjunto de restrições. São discutidos os métodos do tipo Gradiente, Newton e Quase-Newton, com a descrição dos seus algoritmos básicos e apresentação da regra de busca unidimensional adotada (Regra de Armijo ou Exata). Devido ao fato do Método de Newton ter apresentado uma melhor convergência em relação aos demais algoritmos estudados, optou-se por combiná-lo com uma estratégia de conjuntos ativos para o caso de minimização com variáveis canalizadas.

Palavras-chave: Métodos de Otimização, Método da Energia, minimização com variáveis canalizadas, problemas de contato em estruturas. 


\begin{abstract}
RIGO, E. (1999). Linear and Nonlinear Programming applied to structural analysis. São Carlos. 105p. Dissertação (Mestrado). Escola de Engenharia de São Carlos, Universidade de São Paulo.
\end{abstract}

The finite element method when applied to structural analysis, in its usual form, it drives the equations systems that, in the nonlinear case, they demand algorithms repetitive that accomplish, in essence, a linear programming to each load step. However, the Energy Method formulates the problem of structural analysis in the form of the minimizing, could present restrictions on the displacement function, for example. In that case, the algorithms of mathematical programming provide the most consistent way for obtaining of the solution.

The present work negotiates, essentially, of the application in mathematical programming as a form to analyze the nonlinear structures behavior, that can be current of boundary conditions. The structural problems are formulated through Energy Method, that results in the mathematical programming of quadratic functions subject to a group of restrictions. The methods of the type Gradient are discussed, of Newton and Quasi-Newton, with the description of its basic algorithms and presentation of the rule of search adopted unidimensional (Rule of Armijo or Exact). Due to the fact of Newton's Method to have presented a better convergence in relation to the other studied algorithms, it was opted for combining it with a "strategy of the active groups" for the case of mathematical programming with restricted variables.

Keywords: mathematical programming, Energy Method, nonlinear mathematical programming with restricted variables, contact problems in structures. 


\section{CAPÍTULO 1 - INTRODUÇÃO}

A análise de estruturas, com emprego dos métodos numéricos, em computadores, tornou-se, hoje em dia, um procedimento absolutamente comum e instrumento até indispensável para qualquer especialista na área de engenharia de estruturas.

Esse fato associado à evolução muito rápida dos computadores pessoais têm servido de motivação para a pesquisa de novas metodologias empregadas em projetos estruturais e, em particular, o emprego e desenvolvimento de algoritmos numéricos mais robustos e eficientes com vistas à obtenção dos esforços numa estrutura. A tendência atual é de substituição de modelos de cálculo que se baseavam em hipóteses bastante simplificadoras por outros que conseguem representar mais fielmente o comportamento estrutural.

$\mathrm{Na}$ presente pesquisa, através do estudo e emprego de algoritmos de otimização dedicados à minimização de funções sujeitas a restrições, pretende-se dar uma pequena contribuição à análise de problemas estruturais, formulados via Método da Energia. ASSAN (1995)

A relação entre o Método da Energia e os algoritmos de otimização é bastante estreita.

$\mathrm{Na}$ modelagem clássica considerando-se um regime de pequenos deslocamentos e resposta elástica linear do material, procura-se equacionar a energia total envolvida no sistema durante o processo de carregamento e deformação. Tal energia é composta de duas contribuições: uma dita externa, associada ao carregamento, e outra interna, associada à deformação experimentada pelo corpo.

A energia externa é obtida essencialmente pelo trabalho das forças atuantes no corpo, ou seja, dada pelo produto da carga pelo deslocamento do seu ponto de aplicação. A energia interna é obtida através do trabalho das forças de interação entre as partes do corpo, ou seja, dada pelo produto das tensões pelas deformações em todo volume do corpo. 
Assim, somando-se as duas contribuições, ou seja, a energia interna ou de deformação e a energia potencial externa, obtém-se a energia total, aqui representada por $\pi$.

Admitindo-se conhecidos os campos de deslocamentos, pode-se determinar o funcional $\pi$ da energia total para diferentes tipos de elementos estruturais, como os reticulares (barras), planos e tridimensionais.

Um teorema fundamental neste estudo afirma que à situação deformada, em equilíbrio, corresponde um mínimo na energia total. Justamente a imposição desse teorema permite obter os valores incógnitos de deslocamentos e suas derivadas em cada ponto da estrutura.

No Método dos Elementos Finitos, ZIENKIEWICS (1991), a função incógnita deslocamento é aproximada por uma função polinomial conhecida, com a finalidade de se trabalhar com o funcional aproximado da energia. Tal procedimento se baseia numa interpolação sobre um conjunto de pontos, que constituem a estrutura discretizada, e proporciona a obtenção de resultados satisfatórios em casos de condições de carregamento e vinculação mais gerais.

Por outro lado, a minimização do funcional da energia pode ser interpretado como um problema de otimização ou programação matemática, sendo, portanto, indicada a utilização de algoritmos de minimização já desenvolvidos no âmbito daquele campo de estudos. Particularmente no caso dos problemas não-lineares, tais algoritmos passam a ser uma alternativa consistente para a obtenção dos resultados, evitando-se a resolução direta iterativa do sistema de equações. A consistência desses algoritmos se deve ao fato de que atendem a todas as condições matemáticas necessárias para o problema.

Ainda sob o ponto de vista do emprego dos algoritmos de programação matemática, BAZARAA (1979), em combinação com o Método dos Elementos Finitos, a utilização de funções polinomiais aproximadoras e a discretização espacial são muito convenientes, pois permitem exprimir a energia total como função dos valores de deslocamentos e suas derivadas em pontos discretos do domínio da estrutura. Isto vai ao encontro da forma matemática exibida teoricamente pelos problemas de minimização de uma função de $\boldsymbol{n}$ variáveis. Desse modo, a estrutura clássica do Método dos Elementos Finitos é bastante adequada, aproveitando-se toda a parte de geração de funções aproximadoras. 
Com base nos comentários anteriores os objetivos do trabalho ficam mais bem definidos. A motivação é tratar de problemas que consistem da análise de estruturas planas reticuladas, em regime elástico, abordando-se, em particular, a resposta não-linear devido às limitações impostas sobre suas condições de contorno (não-linearidade de contato), as quais são representadas por restrições no modelo matemático. Outro aspecto colocado em destaque no trabalho é uma análise da eficiência dos diferentes algoritmos, dedicados à resolução do problema de minimização.

Entre os algoritmos a serem tratados destacam-se: os procedimentos do tipo Newton e Quase-Newton, combinados com uma estratégia dos conjuntos ativos, e os algoritmos derivados dos métodos do tipo Gradiente.

Os capítulos 2 e 3 desenvolverão basicamente os conceitos matemáticos envolvidos nos métodos de otimização para uma função quadrática. Apenas no capítulo 4 se introduzirá a modelagem de estruturas, via Método dos Elementos Finitos, que discretiza a estrutura espacialmente e gera um funcional aproximado da energia total, cuja minimização será realizada pelos algoritmos do otimização estudados nos capítulos 2 e 3. Esses algoritmos possibilitam a minimização de funções sujeitas a restrições em suas variáveis de interesse. Dessa forma, o funcional da energia a ser analisado poderá conter restrições em seus deslocamentos, como por exemplo, aqueles oriundos de problemas de contato unilateral. Os exemplos de aplicações no capítulo 5 abordarão alguns problemas desse tipo. 


\section{CAPÍTULO 2 - FORMULAÇÃO MATEMÁTICA}

Devido à grande quantidade de problemas físicos e matemáticos, cuja solução corresponde a valores extremos de uma função de interesse, os estudos para o desenvolvimento de estratégias de resolução se dirigem para os métodos de otimização. Tais métodos têm a finalidade de encontrar pontos de máximo ou mínimo locais em funções pré-estabelecidas, sujeitas ou não a um conjunto de restrições.

No presente trabalho estudam-se funções quadráticas do tipo $f(x)=\frac{1}{2} x^{T} H x+S^{T} x+c$. Os algoritmos de otimização abordados são dos tipos Newton e Quase-Newton, combinados com uma estratégia dos conjuntos ativos para variáveis canalizadas, e algoritmos derivados do Método do Gradiente. Estuda-se também, para fins de confronto com tais algoritmos, o método iterativo de GaussSeidel. Um outro procedimento numérico de interesse colocado em destaque é a busca unidimensional adotada para a determinação do passo na direção de descida.

Os problemas de otimização podem ser divididos em dois grupos: problemas restritos e problemas irrestritos, conforme as variáveis de interesse apresentem restrições ou não. Os dois tipos de problemas de otimização serão apresentados neste capítulo.

\section{1 - Problemas Irrestritos}

Problemas de otimização sem restrição são problemas da forma:

$$
\begin{array}{cr}
\text { Minimizar } f(x), \quad x \in \Omega \subseteq R^{n} \\
f: \Omega \rightarrow R & x=\left(\begin{array}{c}
x_{1} \\
x_{2} \\
\vdots \\
x_{n}
\end{array}\right)
\end{array}
$$

Resolver o problema (2.1) consiste em determinar $x^{*} \in \Omega$, tal que: 


$$
f\left(x^{*}\right)<f(x), \quad \forall \mathrm{x} \in \Omega
$$

A solução $x$ *é chamada "solução ótima" (mínimo global).

No caso de $\Omega=R^{n}$ o problema (2.1) é chamado "problema de otimização irrestrita".

O vetor gradiente $(\nabla f)$, é formado pelas primeiras derivadas da função $f$ com relação a cada uma das componentes do vetor $x$.

A hessiana $\left(\nabla^{2} f\right)$ representada em forma de matriz, é formada pelas primeiras derivadas de cada uma das componentes do gradiente com relação a cada uma das componentes do vetor $x$.

Duas condições são necessárias e suficientes para que $x^{*}$ seja um ponto de mínimo local:
i) $\nabla f\left(x^{*}\right)=0$
ii) $\left(x-x^{*}\right)^{T} \nabla^{2} f(x *)\left(x-x^{*}\right)>0$

De fato, desenvolvendo-se $f$, por Taylor, em torno de $x^{*}$, tem-se:

$$
f(x) \cong f\left(x^{*}\right)+\nabla f\left(x^{*}\right)^{T}\left(x-x^{*}\right)+\frac{1}{2}\left(x-x^{*}\right)^{T} \nabla^{2} f\left(x^{*}\right)\left(x-x^{*}\right)+\varphi\left(\|x-x *\|^{2}\right),
$$

onde $\frac{\varphi\left(\|x-x *\|^{2}\right)}{\|x-x *\|^{2}} \rightarrow 0$, quando $x \rightarrow x^{*}$.

Se as condições (2.3) e (2.4) forem verificadas, tem-se:

$$
\begin{aligned}
& f(x) \cong f\left(x^{*}\right)+C \geq f\left(x^{*}\right) \quad \mathrm{x} \approx \mathrm{x}^{*} \\
& \text { onde: } \quad C=\frac{1}{2}\left(x-x^{*}\right)^{T} \nabla^{2} f\left(x^{*}\right)\left(x-x^{*}\right)>0
\end{aligned}
$$

Portanto, $x *$ é um ponto de mínimo local.

Os Métodos de Otimização seguem em geral a seguinte estrutura:

Escolha de $x^{0}$ como solução inicial para o problema que se pretende minimizar ; 
Determinação da direção de descida $d^{K} \in R^{n}$, e do tamanho do passo $\alpha_{K} \in R, \alpha>0$ e pequeno, que se pretende dar na direção de descida, de tal forma que:

$$
f\left(x^{K}+\alpha_{K} d^{K}\right)<f\left(x^{K}\right)
$$

$$
x^{K}+\alpha_{K} d^{K} \text { é chamado solução perturbada de } x^{K} \text { na direção } d^{K} \text {. }
$$

Espera-se que a solução perturbada seja melhor, isto é, tenha um valor menor para a função $f$ (função objetivo).

Quando isto ocorrer, $d^{K}$ é chamada direção de descida, e então:

$$
x^{K+1}=x^{K}+\alpha_{K} d^{K}
$$

Este procedimento deve se repetir até que $\left\|x^{K+1}-x^{K}\right\|<$ tol ou que o número de iterações atinja seu limite máximo. O valor de tol significa uma tolerância pré-estabelecida, sendo geralmente um valor muito pequeno, como por exemplo, tol $=10^{-6}$.

Quando o primeiro critério de parada fôr verificado, significa que o método convergiu e se encontrou $x^{*}$, ou seja, o ponto de mínimo. Caso contrário, se o processo iterativo terminar quando se atingir o número máximo de iterações, significa que o método não convergiu para o determinado problema de minimização em estudo.

\section{2 - Busca Unidimensional}

A Busca Unidimensional trata do seguinte problema: dada uma direção de descida, determinar o tamanho do passo $\alpha$ que encontre o ponto de mínimo da função em estudo nesta direção, isto é:

$$
\begin{array}{cl}
\text { Seja } & \varphi(\alpha)=f\left(x^{0}+\alpha d^{0}\right) \\
& \varphi: \mathrm{R} \rightarrow \mathrm{R}
\end{array}
$$

Encontrar $\min \varphi(\alpha)$ 
A busca unidimensional tem, portanto, a finalidade de determinar o tamanho do passo na direção de descida, ou seja, uma vez determinada uma direção de descida através de um algoritmo de otimização, é preciso que a nova solução encontrada nessa direção esteja o mais próximo possível da solução ótima. Assim sendo, a busca unidimensional representa um importante fator na convergência do método de otimização. As buscas unidimensionais aqui estudas são a Exata e a Aproximada.

\subsection{1 - Exata}

A Busca Unidimensional Exata seria a determinação exata do tamanho do passo de descida segundo a direção desejada. Ela é facilmente aplicada para funções quadráticas da seguinte forma:

$$
f(x)=\frac{1}{2} x^{T} H x+S^{T} x+c,
$$

onde: $H=\nabla^{2} f(x)$ é a matriz Hessiana

$$
S=\nabla^{2} f(0) \quad \text { é o vetor Gradiente em } x=0
$$

O gradiente dessa função é representado por:

$$
\nabla f(x)=H x+S
$$

Como a função é quadrática é possível se determinar o tamanho exato do passo $\alpha$ fazendo-se $\varphi^{\prime}(\alpha)=0$; ou seja:

$$
\varphi^{\prime}(\alpha)=\nabla f^{T}\left(x^{0}+\alpha d^{0}\right) d^{0}=0
$$

Substituindo-se os valores da equação (2.10) em (2.11) tem-se:

$$
\left(H\left(x^{0}+\alpha d^{0}\right)+S\right)^{T} d^{0}=0
$$

Isolando-se a variável $\alpha$ tem-se: 


$$
\alpha=\frac{-S^{T} d_{0}-x_{0}^{T} H d_{0}}{d_{0}^{T} H d_{0}}
$$

\subsection{2 - Aproximada}

Um dos tipos de Busca Aproximada é a Regra de Armijo, que promove a determinação do tamanho do passo $\alpha$ através de uma busca imprecisa.

O problema de se determinar o tamanho do passo na direção de descida continua sendo o mesmo, ou seja:

$$
\begin{gathered}
\text { Seja } \quad \varphi(\alpha)=f\left(x^{0}+\alpha d^{0}\right) \\
\varphi: \quad \mathrm{R} \rightarrow \mathrm{R}
\end{gathered}
$$

Encontrar $\min \varphi(\alpha)$

Resolver (2.15) exatamente pode ser muito custoso. É melhor encontrar um $\bar{\alpha}$ tal que:

$$
\begin{aligned}
& f\left(x^{0}+\bar{\alpha} d^{0}\right)<f\left(x^{0}\right) \quad \text { (Busca imprecisa) } \\
& \text { Como } \quad \varphi(\alpha)=f\left(x^{0}+\alpha d^{0}\right) \quad, \text { então: } \\
& \varphi^{\prime}(\alpha)=\nabla f^{T}\left(x^{0}+\alpha d^{0}\right) d^{0} \\
& \varphi(0)=f\left(x^{0}\right) \\
& \varphi^{\prime}(0)=\nabla f^{T}\left(x^{0}\right) d^{0}
\end{aligned}
$$




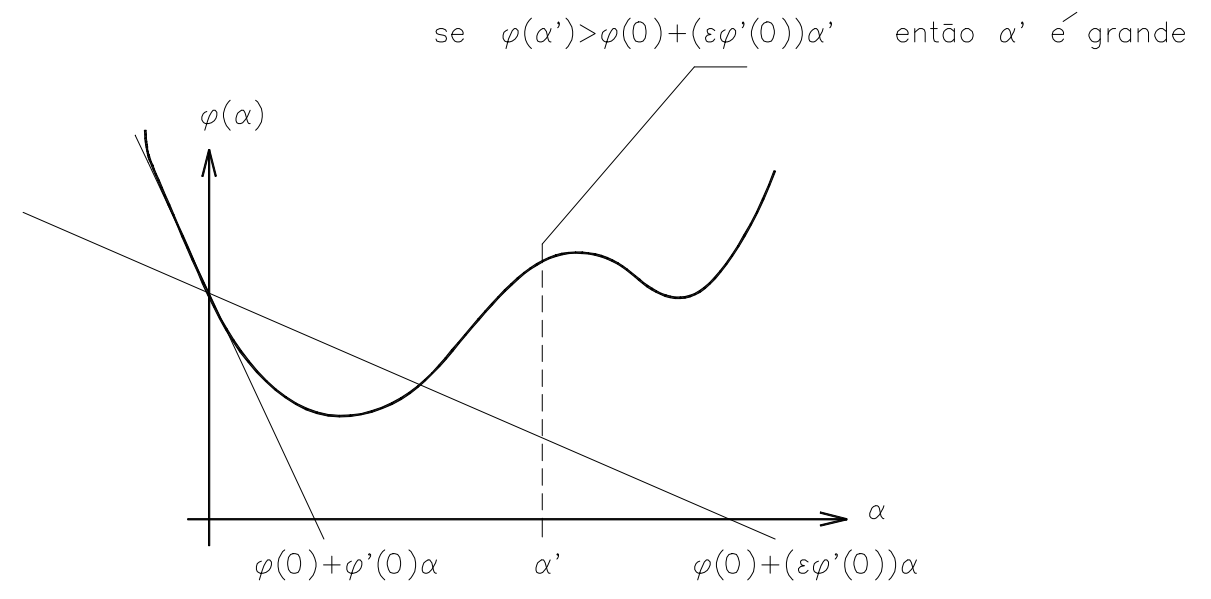

FIGURA 2.1 - Definição gráfica do passo $\alpha$

A Figura 2.1 ilustra a estratégia da Regra de Armijo que consiste inicialmente em se determinar um valor de $\alpha$ que é considerado não tão grande se:

$$
\varphi(\alpha) \leq \varphi(0)+\left(\varepsilon \varphi^{\prime}(0)\right) \alpha
$$

Note que o valor de $\varepsilon$ está compreendido no intervalo $[0,1]$, no entanto neste trabalho o valor de $\varepsilon$ foi adotado como sendo $\varepsilon=0,2$ (sugestão de LUENBERGER (1984)); e a aproximação inicial para o valor de alfa é $\alpha=1$.

Para assegurar que $\alpha$ não seja tão grande, o procedimento adotado é o seguinte:

$$
\alpha \leftarrow \frac{\alpha}{10}
$$

até que a condição (2.21) seja verificada, ou que o número de iterações atinja o seu limite máximo.

Para que o valor de $\alpha$ não seja tão pequeno, multiplica-se $\alpha$ por um $\eta>$ 1 e espera-se que:

$$
\varphi(\eta \alpha)>\varphi(0)+\left(\varepsilon \varphi^{\prime}(0)\right) \eta \alpha
$$


$O$ valor adotado para $\eta$ é $\eta=2$. Portanto o procedimento adotado para que $\alpha$ não seja considerado tão pequeno é:

$$
\alpha \leftarrow 2 \times \alpha
$$

até que a condição (2.23) seja verificada ou que o número de iterações atinja o seu limite máximo.

Assim, encontra-se o valor de $\bar{\alpha}$ através da busca imprecisa, e a condição (2.23) garante que $\varphi(\alpha)=f\left(x^{0}+\alpha d^{0}\right)<f\left(x^{0}\right)=\varphi(0)$, pois $\varphi(\alpha) \leq \varphi(0)+\varepsilon \varphi^{\prime}(0) \alpha<\varphi(0)$, uma vez que $d^{0}$ é escolhido tal que $\varphi^{\prime}(0)=\nabla f^{T}\left(x^{0}\right) d^{0}<0$. A escolha de $d^{0}$ será tratada nas próximas seções.

\section{3 - Métodos de Minimização}

Os três métodos abordados para minimização irrestrita foram os do tipo Gradiente, Newton e Quase-Newton. A essência de cada método está no cálculo de sua direção de descida, que implica diretamente na eficiência e convergência dos mesmos.

Os conceitos básicos desses métodos serão aproveitados e complementados no Capítulo 3 para se tratar de minimização com restrições.

\subsection{1 - Método do Gradiente}

Considere-se o desenvolvimento de $f$, por Taylor, em torno do ponto $x^{K}$ até a $1^{\underline{a}}$ ordem:

$$
f\left(x^{K}+\alpha d^{K}\right)=f\left(x^{K}\right)+\nabla f^{T}\left(x^{K}\right)\left(\alpha d^{K}\right)+\varphi(\alpha),
$$

onde $\varphi(\alpha)$ é tal que $\quad \frac{\varphi(\alpha)}{\alpha} \rightarrow o \quad$ quando $\quad \alpha \rightarrow 0$

Assim:

$$
\frac{f\left(x^{K}+\alpha d^{K}\right)-f\left(x^{K}\right)}{\alpha}=\nabla f^{T}\left(x^{K}\right) d^{K}+\frac{\varphi(\alpha)}{\alpha}
$$


A direção de descida $d^{K}$ deve satisfazer a seguinte condição:

$$
f\left(x^{K}+\alpha d^{K}\right)<f\left(x^{K}\right) \quad \text { com } \alpha>0 \text { e pequeno }
$$

Observando-se a equação (2.26), nota-se que o termo à esquerda será negativo quando o numerador fôr negativo, e pela igualdade, o termo à direita deve ser também negativo, isto é:

$$
\nabla f^{T}\left(x^{K}\right) d^{K}<0
$$

uma vez que $\frac{\varphi(\alpha)}{\alpha}$ torna-se desprezível quando $\alpha$ é pequeno.

Uma escolha para $d^{K}$ que satisfaça (2.28) é:

$$
d^{K}=-\nabla f\left(x^{K}\right),
$$

pois $\quad \nabla f^{T}\left(x^{K}\right) d^{K}=-\left\|\nabla f\left(x^{K}\right)\right\|^{2}<0 \quad$ desde que $\quad \nabla f\left(x^{K}\right) \neq 0$

Portanto, $\quad d^{K}=-\nabla f\left(x^{K}\right)$ satisfaz a condição (2.27), e $d^{K}$ é uma direção de descida.

Isto define o "Método do Gradiente".

\subsection{2 - Método de Newton}

O Método de Newton consiste em:

i) desenvolver a função $\boldsymbol{f}$, por Taylor, em torno de um ponto $x^{K}$, até a $2^{\underline{a}}$ ordem obtendo-se uma aproximação quadrática. 
ii) A nova solução é obtida pelo mínimo da quadrática

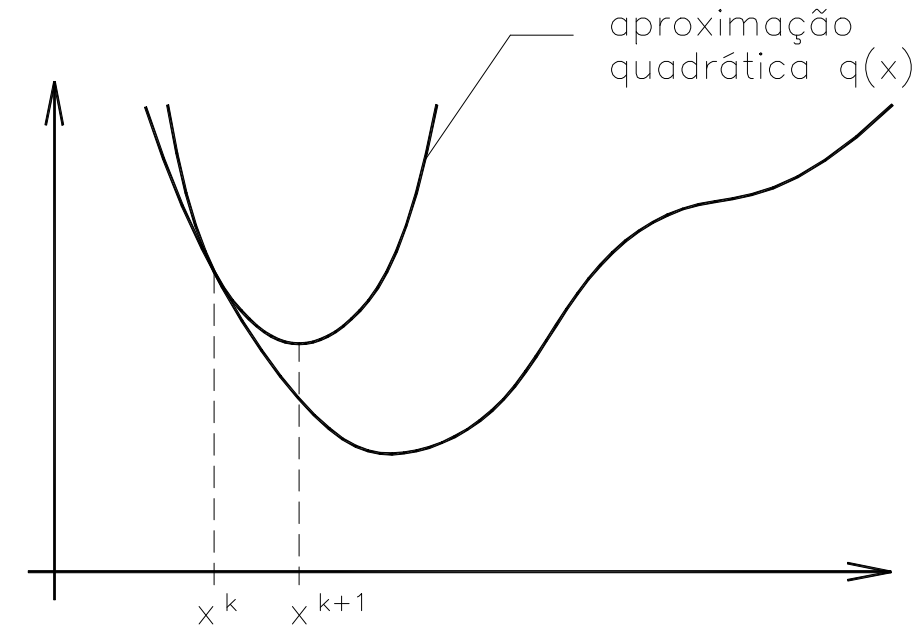

FIGURA 2.2 - Aproximação por uma função quadrática pelo Método de Newton

Aproximação de Taylor para função quadrática:

$$
\begin{aligned}
f(x) \cong & f\left(x^{K}\right)+\nabla f^{T}\left(x^{K}\right)\left(x-x^{K}\right)+ \\
& +\frac{1}{2}\left(x-x^{K}\right)^{T} \nabla^{2} f\left(x^{K}\right)\left(x-x^{K}\right)=q(x)
\end{aligned}
$$

O ponto $x^{K+1}$ é determinado pelo mínimo de $q(x)$.

Determina-se primeiramente $\nabla q(x)$ :

$$
\nabla q(x)=\nabla f\left(x^{K}\right)+\nabla^{2} f\left(x^{K}\right)\left(x-x^{K}\right)
$$

Igualando-se a zero, tem-se:

$$
\nabla q(x)=0 \Leftrightarrow \nabla^{2} f\left(x^{K}\right)\left(x-x^{K}\right)=-\nabla f\left(x^{K}\right)
$$

Hipótese: $\exists$ inversa de $\nabla^{2} f\left(x^{K}\right)$ 
Assim, multiplicando-se por $\left[\nabla^{2} f\left(x^{K}\right)\right]^{-1}$, tem-se:

$$
x-x^{K}=-\left[\nabla^{2} f\left(x^{K}\right)\right]^{-1} \nabla f\left(x^{K}\right),
$$

e a solução de $\nabla q(x)=0$ é dada por:

$$
x^{K+1} \equiv x=x^{K}-\left[\nabla^{2} f\left(x^{K}\right)\right]^{-1} \nabla f\left(x^{K}\right)
$$

Este é o Método de Newton "puro".

Nota-se que:

$$
x^{K+1}=x^{K}+d^{K} ; \text { onde } \quad d^{K}=-\left[\nabla^{2} f\left(x^{K}\right)\right]^{-1} \nabla f\left(x^{K}\right)
$$

Na prática, faz-se:

$$
x^{K+1}=x^{K}+\alpha_{K} d^{K},
$$

onde o passo $\alpha_{K}$ é determinado como anteriormente, tal que:

$$
f\left(x^{K}+\alpha_{K} d^{K}\right)<f\left(x^{K}\right)
$$

Para o cálculo de direção de descida $d^{K}$, é computacionalmente mais eficiente resolver o sistema linear abaixo:

$$
\left\lfloor\nabla^{2} f\left(x^{K}\right)\right\rfloor d^{K}=-\nabla f\left(x^{K}\right)
$$

Isto define o "Método de Newton". 


\subsection{3 - Método de Quase-Newton}

Os Métodos de Quase-Newton derivam do Método de Newton, o qual, como se mostrou, consiste em admitir que nas vizinhanças do ponto de mínimo, a função é quadrática. Assim é conveniente reproduzir as duas relações que resumem o Método de Newton:

$$
f(x) \cong f\left(x^{K}\right)+\nabla f^{T}\left(x^{K}\right)\left(x-x^{K}\right)+\frac{1}{2}\left(x-x^{K}\right)^{T} \nabla^{2} f\left(x^{K}\right)\left(x-x^{K}\right)
$$

Impondo-se $\nabla f(x)=0$ no ponto de mínimo, resulta que:

$$
x^{K+1}=x^{K}-\left[\nabla^{2} f\left(x^{K}\right)\right]^{-1} \nabla f\left(x^{K}\right)
$$

Os Métodos de Quase-Newton aproximam a inversa da hessiana, aqui representada por $Y$, introduzindo também a busca unidimensional, inexistente no Método de Newton puro. Assim, uma melhor aproximação para o ponto de mínimo fica expressa por:

$$
x^{K+1}=x^{K}-Y_{K} \nabla f\left(x^{K}\right)
$$

Como a matriz hessiana é representada por $H_{K}=\nabla^{2} f\left(x^{K}\right)$, sua inversa fica:

$$
Y_{K}=\left[H_{K}\right]^{-1}
$$

Entretanto, $H_{K}$ deve verificar a equação característica do Método de Newton:

$$
\left[H_{K}\right]^{-1} q_{K}=p_{K}
$$

onde:

$$
q_{K}=\nabla f\left(x^{K+1}\right)-\nabla f\left(x^{K}\right) \quad \text { e } \quad \mathrm{p}_{\mathrm{K}}=\mathrm{x}^{\mathrm{K}+1}-\mathrm{x}^{\mathrm{K}}
$$

Uma regra de aproximação da Hessiana estudada foi a proposta por Davidon-Fletcher-Powell (DFP), LUENBERGER (1984). 
Essa regra utiliza um procedimento recursivo para a obtenção de $Y_{K}$, constituindo-se também num método de direções conjugadas.

Em cada etapa de um procedimento iterativo, $Y_{K}$ é atualizada através de uma relação recursiva do tipo:

$$
Y_{K+1}=Y_{K}+a_{K} z_{K} z_{K}^{T} \quad \text { onde } a_{K} z_{K} z_{K}^{T} \quad \text { é uma correção em } H_{K} \quad \text { tal }
$$

que: $\quad Y_{K+1} q_{K}=p_{K}$

A expressão final é dada por:

$$
Y_{K+1}=Y_{K}+\frac{\left(p_{K}-Y_{K} q_{K}\right)\left(p_{K}-Y_{K} q_{K}\right)^{T}}{q_{K}^{T}\left(p_{K}-Y_{K} q_{K}\right)}
$$

O Método DFP apresenta o seguinte algoritmo:

Passo inicial) escolha $Y_{0}$ simétrica positiva definida e $x^{0}$ um ponto qualquer;

Passo 1) $d^{K}=-Y_{K} q_{K}$, onde $q_{K}=\nabla f\left(x^{K}\right)$;

Passo 2) $x^{K+1}=x^{K}+\alpha_{K} d^{K}$, sendo $\alpha_{K}$ tal que minimize $f\left(x^{K}+\alpha_{K} d^{K}\right)$

$$
p_{K}=\alpha_{K} d^{K} \quad \text { e } \quad q_{K+1}=\nabla f\left(x^{K+1}\right) ;
$$

Passo 3) $q_{K}=q_{K+1}-q_{K}$

$$
Y_{K+1}=Y_{K}+\frac{p_{K} p_{K}^{T}}{p_{K}^{T} q_{K}}-\frac{Y_{K} q_{K} q_{K}^{T} Y_{K}}{q_{K}^{T} Y_{K} q_{K}} ; \quad K=K+1 \text { e volte para o passo } 1
$$




\section{CAPÍTULO 3 - PROBLEMAS COM VARIÁVEIS CANALIZADAS}

Os problemas de otimização com restrições que se estudam neste trabalho são problemas da seguinte forma:

$$
\text { Minimizar } f(x), x \in \Omega \subseteq R^{n},
$$

onde $\Omega=\left\{x \in R^{n} / a \leq x \leq b\right\}$

Nesse problema $f$ é uma função de valor escalar, isto é, $f: \Omega \rightarrow R$, e os vetores $x, a, b \in R^{n}$ podem ser representados nas formas:

$$
x=\left(\begin{array}{c}
x_{1} \\
x_{2} \\
\vdots \\
x_{n}
\end{array}\right) \quad a=\left(\begin{array}{c}
a_{1} \\
a_{2} \\
\vdots \\
a_{n}
\end{array}\right) \quad b=\left(\begin{array}{c}
b_{1} \\
b_{2} \\
\vdots \\
b_{n}
\end{array}\right) \text {, }
$$

onde $a$ e $b$ significam as restrições nas variáveis da função que se pretende minimizar.

Resolver o problema (3.1) consiste em determinar $x^{*} \in \Omega$ tal que:

$$
f\left(x^{*}\right) \leq f(x), \quad \forall x \in \Omega
$$

A solução $x^{*}$ é chamada "solução ótima" para o problema de otimização com restrição. Quando $\Omega=R^{n}$ o problema (3.1) é chamado de "problema de minimização irrestrita". Nota-se que o mínimo irrestrito da função $f$ pode não pertencer ao conjunto $\Omega$.

As condições necessárias de mínimo irrestrito eram: $\nabla f(x *)=0$, entretanto em função das restrições, tais condições podem não ser necessariamente verificadas. A figura (3.1), com $n=1$, ilustra esta situação. 

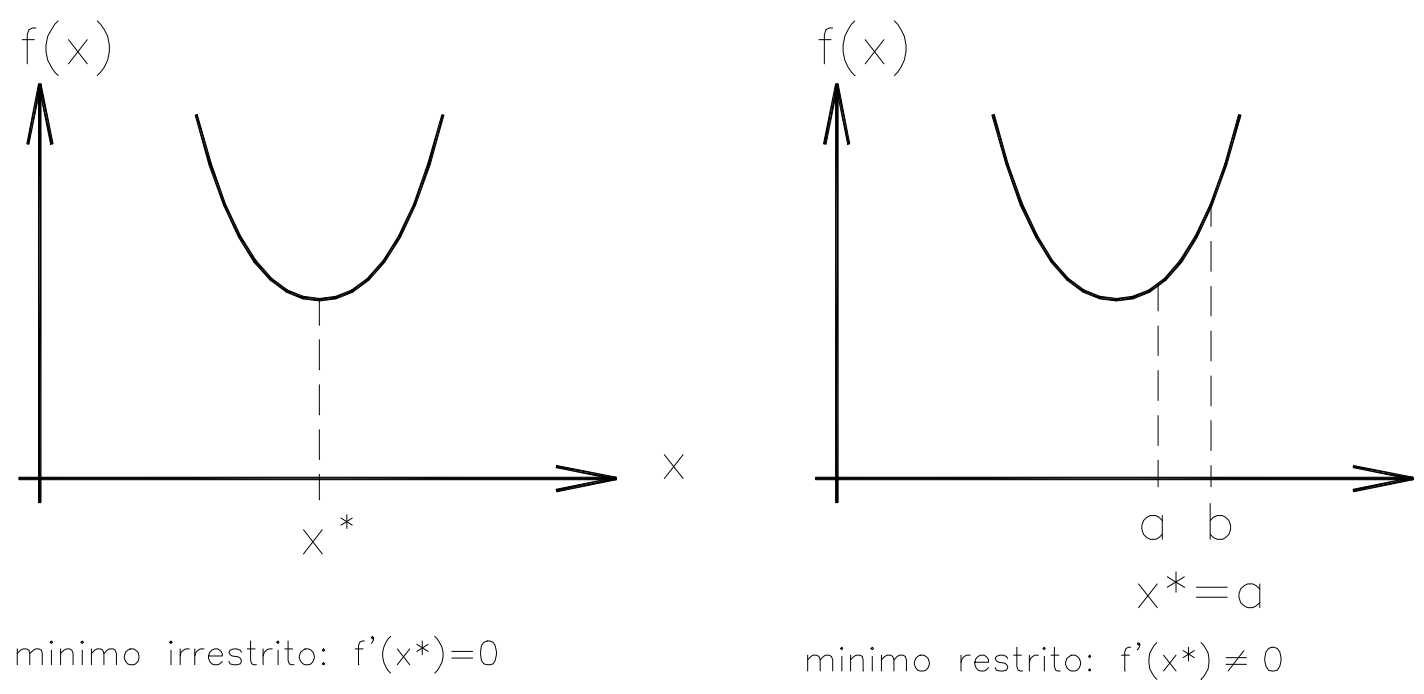

FIGURA 3.1 - Pontos de mínimos restrito e irrestrito

As condições necessárias de $1^{\underline{a}}$ ordem para os problemas de otimização serão estudados no item (3.2).

Uma característica básica dos métodos de minimização com restrições é que a solução inicial aproximada $x^{0}$ deve pertencer à região $\Omega$, denominada região de factibilidade. Dessa forma, como $x^{0} \in \Omega$, então $x^{0}$ recebe o nome de solução factível.

Os métodos para otimização com restrições seguem em geral a seguinte estrutura:

1. Escolha de $x^{0} \in \Omega$ como solução inicial aproximada para o problema que se pretende minimizar. Toma-se $K=0$;

2. Determinação em $x^{K}$ da direção de descida factível ${ }^{1}, d^{K} \in R^{n}$;

3. Determinação do tamanho do passo [M1] $\alpha_{K} \in R, \alpha>0$ e pequeno, que se pretende dar na direção de descida factível, de tal forma que a solução perturbada seja melhor, isto é, tenha um valor menor para a função $f$ :

$$
f\left(x^{K}+\alpha_{K} d^{K}\right)<f\left(x^{K}\right)
$$

\footnotetext{
${ }^{1}$ Direção de descida factível significa que a direção é de descida (o valor da função $\mathrm{f}(\mathrm{x})$ está decrescendo naquela direção) e que a direção é factível (nessa direção existem novas soluções para o problema, pertencentes à região de factibilidade). Estes conceitos básicos serão estudados com mais detalhes logo a seguir.
} 
Na relação anterior, $x^{K}+\alpha_{K} d^{K}$ é chamado solução perturbada de $x^{K}$ na direção $d^{K}$ com passo $\alpha_{K}$;

4. Calcula-se uma nova solução:

$$
\begin{aligned}
& x^{K+1}=x^{K}+\alpha_{K} d^{K} \\
& K \leftarrow K+1 ;
\end{aligned}
$$

5. Os passos 2, 3 e 4 devem se repetir até que $\left\|x^{K+1}-x^{K}\right\|<$ tol ou que o número de iterações atinja um limite máximo estabelecido ( $\mathrm{K}=$ limite). $\mathrm{O}$ valor de $t o l$ significa uma tolerância pré-estabelecida, sendo geralmente um valor muito pequeno, como por exemplo, tol $=10^{-6}$.

Quando o primeiro critério de parada fôr verificado, significa que o método convergiu e se encontrou $x^{*}$, ou seja, o ponto de mínimo. Caso contrário, se o processo iterativo terminar quando se atingir o número máximo de iterações, significa que o método não convergiu para o determinado problema de minimização com restrições em estudo.

Outros critérios de parada, mais pertinentes ao problema de otimização, podem ser facilmente incorporados, como por exemplo, interromper o procedimento iterativo quando:

$$
f\left(x^{K}\right)-f\left(x^{K+T}\right)<\operatorname{tol}_{2}
$$

isto é, após $T$ iterações do método, a melhoria na função objetivo observada foi inferior a uma tolerância $\mathrm{tol}_{2}$.

Nota-se que a diferença entre os métodos de otimização com restrições para os métodos de otimização irrestritos está na determinação da direção $d^{K}$, que além de ser uma direção de descida deve ser uma direção factível.

\section{1 - Direção Factível}

Se $x^{K}$ for uma solução factível e $d^{K} \in R^{n}$ tal que $\left(x^{K}+\alpha d^{K}\right)$ também seja uma solução factível para $\alpha>0$ e pequeno, pode-se dizer que $d^{K}$ é uma direção 
factível em $x^{K}$. A figura (3.2) ilustra uma direção factível em $\bar{x} \in \Omega$, porém a mesma direção não é factível em $x \in \Omega$.

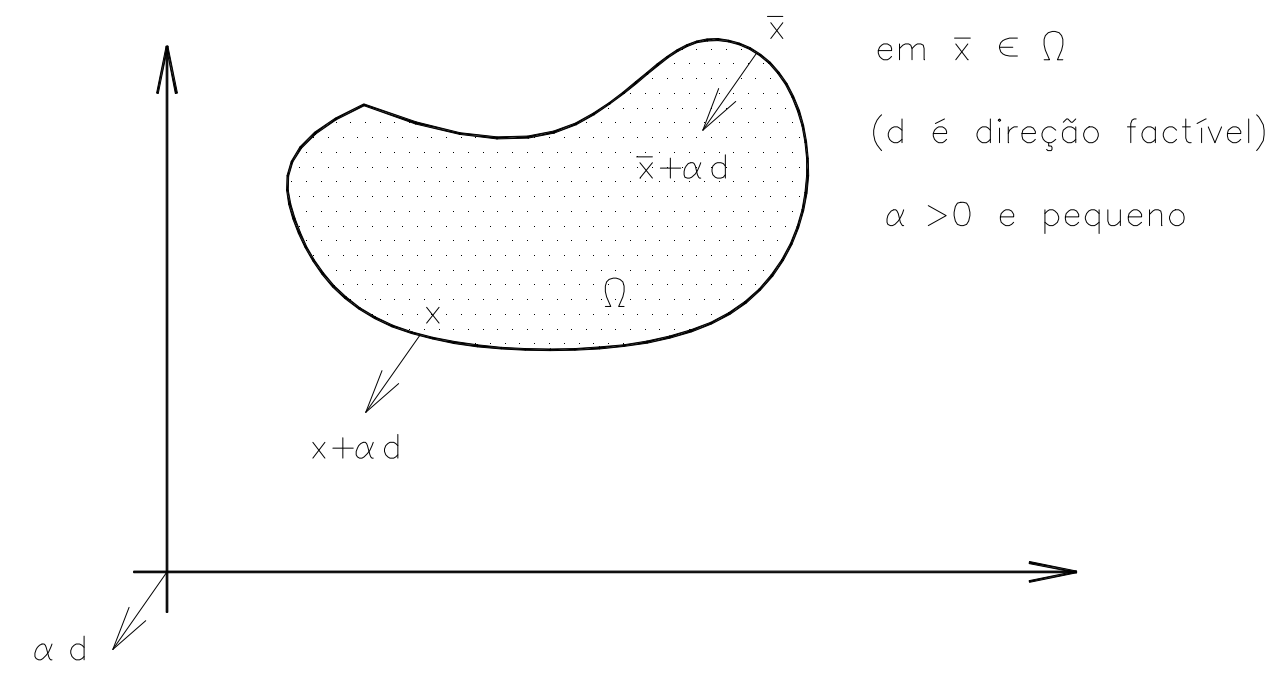

FIGURA 3.2 - Direção factível em $\bar{x}$

Mais formalmente, a definição de direção factível é dada por:

Seja $\bar{x} \in \Omega \subseteq R^{n}$, diz-se que $d \in R^{n}$ é uma direção factível em $\bar{x}$, se $\exists \alpha>0$ tal que: $x=(\bar{x}+\alpha d) \in \Omega \quad \forall \alpha \in(0, \bar{\alpha}]$.

Exemplo:

Em $\Omega=\left\{x \in R^{2} / 0 \leq x_{i} \leq 2\right\}$, considere-se $\bar{x}=\left(\begin{array}{l}2 \\ 1\end{array}\right) \in \Omega$. Esses dados estão ilustrados na figura (3.3):

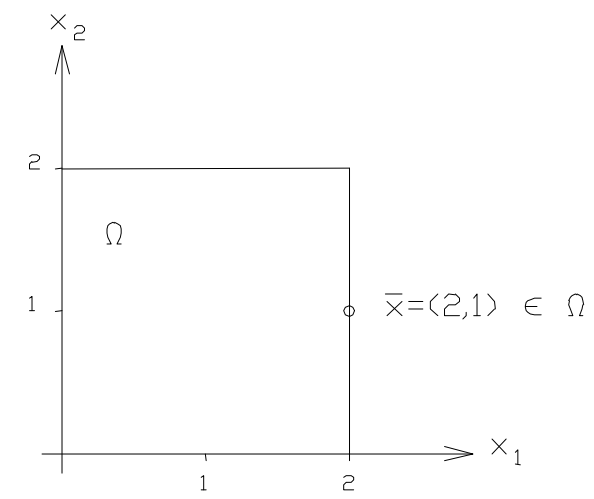

FIGURA 3.3 - Região de factibilidade

Note que $\bar{x}_{1}=2$ (limite superior) e $0<\bar{x}_{2}<2$. 
As direções factíveis em $\bar{x}$, podem ser obtidas por: $d=\left(\begin{array}{l}d_{1} \\ d_{2}\end{array}\right)$ tal que:

$\bar{x}+\alpha d \in \Omega$ ou

$\left(\begin{array}{l}2 \\ 1\end{array}\right)+\alpha\left(\begin{array}{l}d_{1} \\ d_{2}\end{array}\right) \in \Omega$

Pela definição de $\Omega$ obtém-se:

$0 \leq 2+\alpha d_{1} \leq 2 \Rightarrow-2 \leq \alpha d_{1} \leq 0 \Rightarrow d_{1} \leq 0$

$0 \leq 1+\alpha d_{2} \leq 2 \Rightarrow-1 \leq \alpha d_{2} \leq 1 \Rightarrow \forall d_{2}$

Uma escolha possível é $d=\left(\begin{array}{c}-1 \\ 0\end{array}\right)$ e, nesse caso, o passo máximo é $\alpha=2$.

A figura (3.4) ilustra esta escolha.

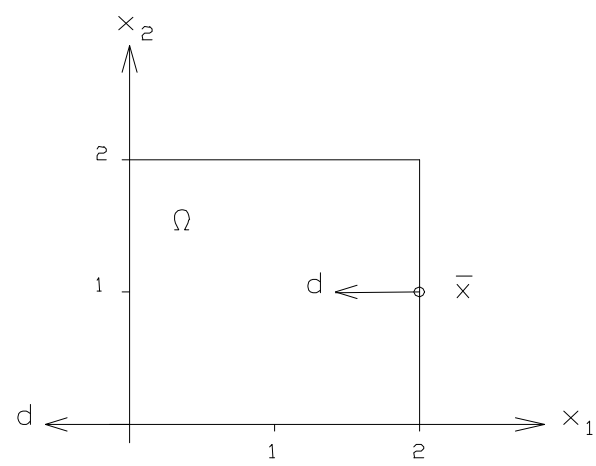

FIGURA 3.4 - Uma direção factível possível

Numa outra situação, $\operatorname{com} \hat{x}=\left(\begin{array}{l}0 \\ 0\end{array}\right) \in \Omega$, então as direções factíveis em $\hat{x}$, $d=\left(\begin{array}{l}d_{1} \\ d_{2}\end{array}\right)$, são tais que: $d_{1} \geq 0, d_{2} \geq 0$.

\section{2 - Direção de Descida}

Seja $\bar{x} \in \Omega \subseteq R^{n}$ e $d \in R^{n}$ uma direção factível em $\bar{x}$. Diz-se que $d$ é uma direção de descida em $\bar{x}$ se $\exists \alpha>0$ tal que: 


$$
f(\bar{x}+\alpha d)<f(\bar{x}) \quad \forall \alpha \in(0, \bar{\alpha}]
$$

Considere-se o desenvolvimento de $f$, por Taylor, em torno do ponto $\bar{x}$ (suposto $f$ diferenciável):

$$
f(\bar{x}+\alpha d)=f(\bar{x})+\nabla f^{T}(\bar{x})(\alpha d)+\varphi(\alpha),
$$

onde $\varphi(\alpha)$ é tal que $\quad \frac{\varphi(\alpha)}{\alpha} \rightarrow 0$, quando $\alpha \rightarrow 0$

Assim, $\operatorname{com} \alpha>0$ :

$$
\frac{f(\bar{x}+\alpha d)-f(\bar{x})}{\alpha}=\nabla f^{T}(\bar{x}) d+\frac{\varphi(\alpha)}{\alpha}
$$

Como se procura uma direção de descida, o termo à esquerda em (3.8) deve ser negativo, e pela igualdade, o termo à direita deve ser negativo também, isto é:

$$
\nabla f(\bar{x}) \cdot d<0
$$

uma vez que $\frac{\varphi(\alpha)}{\alpha}$ torna-se desprezível quando $\alpha$ é pequeno, supondo-se, naturalmente, que $\nabla f(\bar{x}) \cdot d \neq 0$. A recíproca é também verdadeira, isto é, a condição (3.9) garante que $d$ é uma direção de descida em $\bar{x}$.

Portanto, a direção de descida é encontrada resolvendo-se a condição (3.9), ou seja, como:

$$
\nabla f=\left(\begin{array}{llll}
\frac{\partial f}{\partial x_{1}} & \frac{\partial f}{\partial x_{2}} & \cdots & \frac{\partial f}{\partial x_{n}}
\end{array}\right) \in R^{n},
$$

então:

$$
\nabla f^{T} d=\frac{\partial f}{\partial x_{1}} d_{1}+\frac{\partial f}{\partial x_{2}} d_{2}+\cdots+\frac{\partial f}{\partial x_{n}} d_{n}<0
$$

Das observações anteriores, pode-se provar os seguintes teoremas: 


\section{Teorema:}

Sejam $f: \Omega \subseteq R^{n} \rightarrow R$ e $f \in C^{1}$ (funções diferenciáveis) e $d$ uma direção factível em $\bar{x} \in \Omega$. Se:

$\nabla f(\bar{x}) \cdot d<0 \Rightarrow d$ é uma direção de descida

Teorema: (condição necessária de $1^{\underline{a}}$ ordem)

Seja $\bar{x} \in \Omega$ e $f: \Omega \subseteq R^{n} \rightarrow R, f \in C^{1}$

Se $\bar{x}$ for ponto de mínimo de $\mathrm{f}$ em $\Omega$ então:

$\nabla f(\bar{x}) \cdot d \geq 0 \quad \forall d$, direção factível

Retornando-se ao problema de otimização, ou seja, minimizar $f(\bar{x})$ com $\bar{x} \in \Omega=\left\{\bar{x} \in R^{n} / a_{i} \leq \bar{x}_{i} \leq b_{i}\right\}$, numa certa situação genérica alguns componentes de $\overline{\mathrm{X}}$ podem estar em seus limites inferiores, superiores ou entre os limites:

$$
\bar{x}=\left(\begin{array}{c}
a_{1} \\
\vdots \\
a_{k} \\
--b_{k+1} \\
\vdots \\
b_{r} \\
--- \\
\bar{x}_{r+1} \\
\vdots \\
\bar{x}_{n}
\end{array}\right) \quad a_{j}<\bar{x}_{j}<b_{j}, \quad j=r+1, \ldots, n
$$

Isto significa que os valores de $\bar{x}_{i}$ para $\mathbf{i}=\mathbf{1}, \ldots ., \mathbf{k}$ estão no limite inferior; os valores de $\bar{x}_{i}$ para $\mathbf{i}=\mathbf{k}+\mathbf{1}, \ldots, \mathbf{r}$ estão no limite superior e os valores de $\bar{x}_{i}$ para $\mathbf{i}=$ $\mathbf{r}+\mathbf{1}, \ldots, \mathbf{n}$ estão entre os limites.

Uma direção factível em $\bar{x}$ deve ser: 


$$
d=\left(\begin{array}{c}
d_{1} \\
\vdots \\
d_{k} \\
-d_{k+1} \\
\vdots \\
d_{r} \\
-d_{r+1} \\
\vdots \\
d_{n}
\end{array}\right)
$$

tal que:

$d_{i} \geq 0$ para $\mathbf{i}=\mathbf{1}, \ldots, \mathbf{k}$;

$d_{i} \leq 0$ para $\mathbf{i}=\mathbf{k}+\mathbf{1}, \ldots, \mathbf{r}$;

quaisquer para $\mathbf{i}=\mathbf{r}+\mathbf{1}, \ldots, \mathbf{n}$.

Procura-se uma direção de descida:

$$
\begin{aligned}
& \nabla f(\bar{x}) . d<0 \quad \text { ou } \\
& \frac{\partial f(\bar{x})}{\partial x_{1}} d_{1}+\frac{\partial f(\bar{x})}{\partial x_{2}} d_{2}+\cdots+\frac{\partial f(\bar{x})}{\partial x_{k}} d_{k}+\frac{\partial f(\bar{x})}{\partial x_{k+1}} d_{k+1}+ \\
& +\cdots+\frac{\partial f(\bar{x})}{\partial x_{r}} d_{r}+\frac{\partial f(\bar{x})}{\partial x_{r+1}} d_{r+1}+\cdots+\frac{\partial f(\bar{x})}{\partial x_{n}} d_{n}<0
\end{aligned}
$$

O Método do Gradiente (variáveis irrestritas) sugere a seguinte escolha para a direção $d$ :

$$
d=-\nabla f(\bar{x}),
$$

pois $\quad \nabla f(\bar{x}) \cdot d=-\|\nabla f(\bar{x})\|^{2}<0 \quad$ desde que $\quad \nabla f(\bar{x}) \neq 0$

Portanto, $d=-\nabla f(\bar{x})$ satisfaz (3.9), então $d$ é uma direção de descida. No entanto, $d$ assim definida pode não ser uma direção factível. Para se garantir que $d$ seja uma direção de descida factível deve-se observar que a variável que já atingiu seu 
limite inferior ou superior na direção de descida venha a ter sinal adequado (conforme direção factível já estudada), pois assim, em nenhum momento do processo iterativo de minimização essa variável sairá da região de factibilidade. Com isso, um tipo de direção de descida factível $d$ é dada por:

Para $\mathbf{i}=\mathbf{1}, \ldots, \mathbf{k}$ tem-se:

$d_{i}= \begin{cases}-\frac{\partial f(\bar{x})}{\partial x_{i}}, & \text { se } \frac{\partial f(\bar{x})}{\partial x_{i}}<0 \\ 0, \quad \text { caso } & \text { contrário }\end{cases}$

Para $\mathbf{i}=\mathbf{k}+\mathbf{1}, \ldots, \mathbf{r}$ tem-se:

$d_{i}= \begin{cases}-\frac{\partial f(\bar{x})}{\partial x_{i}}, & \text { se } \frac{\partial f(\bar{x})}{\partial x_{i}}>0 \\ 0, \quad \text { caso } & \text { contrário }\end{cases}$

Para $\mathbf{i}=\mathbf{r}+\mathbf{1}, \ldots, \mathbf{n}$ tem-se:

$$
d_{i}=-\frac{\partial f(\bar{x})}{\partial x_{i}}
$$

O tamanho do passo $\alpha$ pequeno na direção de descida factível é tomado como o menor valor encontrado entre o máximo valor de $\alpha$ para não se sair da região de factibilidade $(\bar{\alpha})$ e a resolução do problema de minimização unidimensional através da "busca" Exata ou Aproximada $\left(\alpha_{\text {busca }}\right)$, ou seja:

$$
\begin{aligned}
& \alpha=\min \left\{\alpha_{\text {busca }}, \bar{\alpha}\right\} \\
& \text { onde } \quad \bar{\alpha}=\min \left\{\bar{\alpha}_{1}, \bar{\alpha}_{2}, \cdots, \bar{\alpha}_{n}\right\}
\end{aligned}
$$

Os três casos possíveis para determinação de $\bar{\alpha}_{i}$ são: 
i) $\bar{x}_{i}=a_{i}$

$\left\{\begin{array}{l}\text { se } \frac{\partial f(\bar{x})}{\partial x_{i}}<0 \Rightarrow \hat{d}_{i}>0 \rightarrow \bar{\alpha}_{i}=\frac{b_{i}-a_{i}}{d_{i}} \\ \text { se } \frac{\partial f(\bar{x})}{\partial x_{i}}>0 \Rightarrow \hat{d}_{i}=0 \rightarrow \therefore \forall \bar{\alpha}_{i}\end{array}\right.$

ii) $\bar{x}_{i}=b_{i}$

$\left\{\begin{array}{l}\text { se } \frac{\partial f(\bar{x})}{\partial x_{i}}>0 \Rightarrow \hat{d}_{i}<0 \rightarrow \bar{\alpha}_{i}=\frac{a_{i}-b_{i}}{d_{i}} \\ \text { se } \frac{\partial f(\bar{x})}{\partial x_{i}}<0 \Rightarrow \hat{d}_{i}=0 \rightarrow \therefore \forall \bar{\alpha}_{i}\end{array}\right.$

iii) $a_{i}<\bar{x}_{i}<b_{i}$

$\left\{\begin{array}{l}\text { se } \frac{\partial f(\bar{x})}{\partial x_{i}}>0 \Rightarrow \hat{d}_{i}<0 \rightarrow \bar{\alpha}_{i}=\frac{a_{i}-x_{i}}{d_{i}} \\ \text { se } \frac{\partial f(\bar{x})}{\partial x_{i}}<0 \Rightarrow \hat{d}_{i}>0 \rightarrow \bar{\alpha}_{i}=\frac{b_{i}-x_{i}}{d_{i}}\end{array}\right.$

Um teorema importante deve ser observado para os casos em que $\hat{d}=0$ :

\section{$\underline{\text { Teorema: }}$}

Se $\hat{d}=0$ então $\nabla f(\bar{x}) \cdot d \geq 0$ para $\forall d$ que seja direção factível, sendo que $\bar{x} \in \Omega$ e $\Omega=\left\{x \in R^{n} / a \leq x \leq b\right\}$, ou seja, se $\hat{d}=0$, é inútil procurar direções factíveis tais que $\nabla f(\bar{x}) \cdot d<0$.

\section{Prova:}

Seja $d$ (direção factível) e $\nabla f(\bar{x}) \cdot d<0 \Rightarrow$ existe uma parcela tal que:

$$
\frac{\partial f(\bar{x})}{\partial x_{i}} d_{i}<0
$$

Existem três possibilidades:

$$
\left\{\begin{array}{l}
\bar{x}_{i}=a_{i} \\
\bar{x}_{i}=b_{i} \\
\bar{x}_{i} \in\left(a_{i}, b_{i}\right)
\end{array}\right.
$$


Assim,

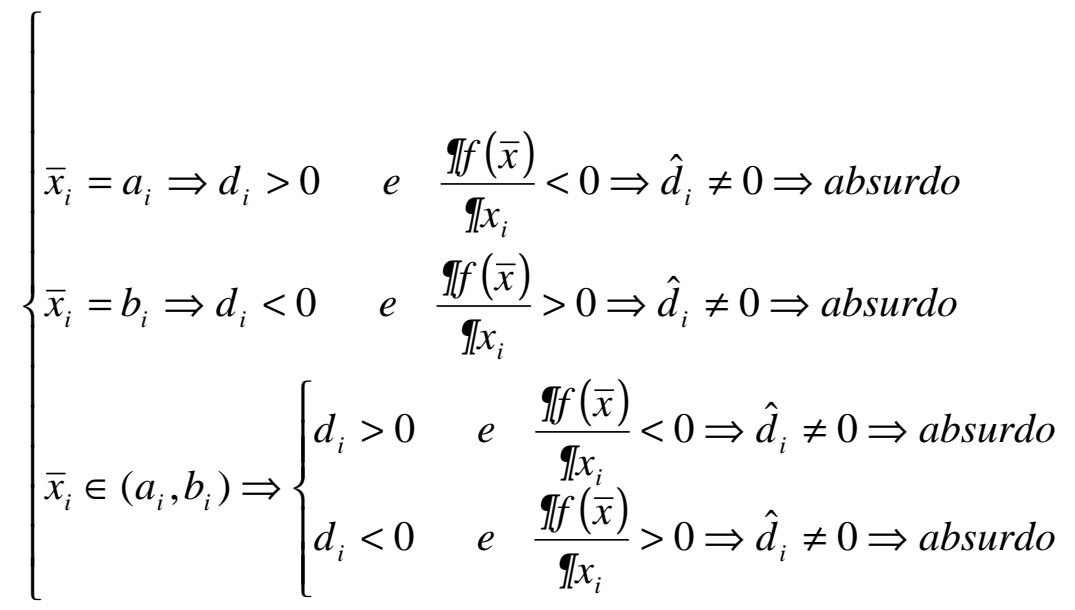

Portanto, $\nabla f(\bar{x}) d \geq 0, \quad \forall d$ : direção factível em $\bar{x}$. Daí concluí-se que $\bar{x} \in \Omega$ é a solução ótima.

\section{Exemplo}

$$
\begin{aligned}
& \min f\left(x_{1}, x_{2}\right)=\left(x_{1}-3\right)^{2}+x_{2}^{2} \\
& x \in \Omega=\left\{x \in R^{n} / 0 \leq x_{i} \leq 2\right\} \\
& \text { Seja: } \quad \bar{x}=\left(\begin{array}{l}
2 \\
1
\end{array}\right)
\end{aligned}
$$

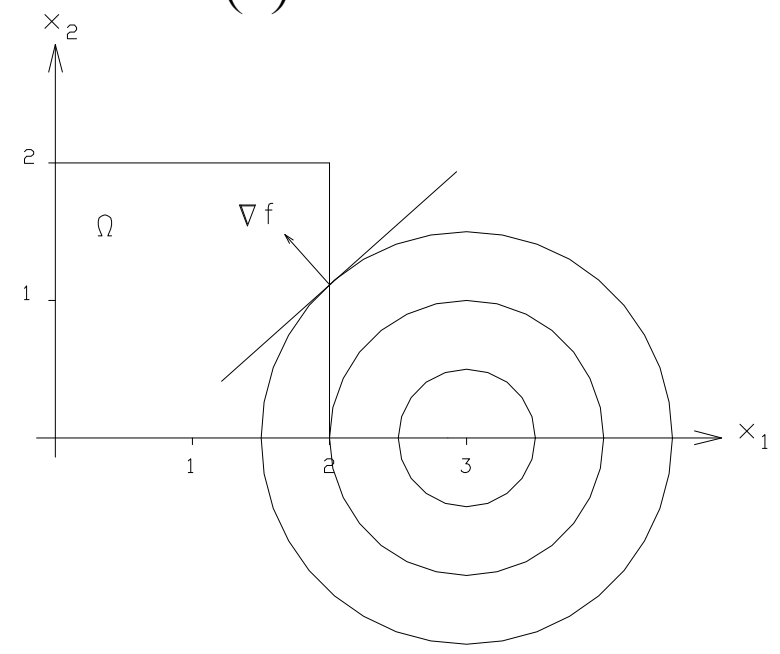

FIGURA 3.5 - $\nabla \mathbf{f}$ na solução factível

Note que se $f=1$ tem-se a equação da circunferência (curva de nível da função objetivo). 


$$
\begin{aligned}
& \nabla f=\left\{2\left(x_{1}-3\right) \quad 2 x_{2}\right\} \\
& \nabla f(\bar{x})=\left\{\begin{array}{ll}
-2 & 2
\end{array}\right\}
\end{aligned}
$$

$$
\text { se } \beta>\frac{\pi}{2}, \nabla f(\bar{x}) \cdot d=\|\nabla f(\bar{x})\|\|d\| \cos \beta<0
$$

Note que a direção do Método do Gradiente, $d=-\nabla f(\bar{x})=\left(\begin{array}{ll}2 & -2\end{array}\right)$, não é uma direção factível, embora:

$$
\begin{aligned}
& \nabla f(\bar{x}) \cdot d=\frac{\partial f(\bar{x})}{\partial x_{1}}\left(-\frac{\partial f(\bar{x})}{\partial x_{1}}\right)+\frac{\partial f(\bar{x})}{\partial x_{2}}\left(-\frac{\partial f(\bar{x})}{\partial x_{2}}\right)= \\
& =-\left[\left(\frac{\partial f(\bar{x})}{\partial x_{1}}\right)^{2}+\left(\frac{\partial f(\bar{x})}{\partial x_{2}}\right)^{2}\right]<0
\end{aligned}
$$

As direções factíveis em $\bar{x}$ devem satisfazer $d_{1} \leq 0 \quad$ e $\quad d_{2}$ qualquer.

Deseja-se que:

$$
\frac{\partial f(\bar{x})}{\partial x_{1}} d_{1}+\frac{\partial f(\bar{x})}{\partial x_{2}} d_{2}<0, \text { ou seja: } \quad-2 d_{1}+2 d_{2}<0
$$

Uma escolha possível é dada por $\hat{\mathrm{d}}$ :

$$
\begin{aligned}
& \hat{d}_{1}=0 \\
& \hat{d}_{2}=-\frac{\partial f(\bar{x})}{\partial x_{2}}=-2 \quad \text { ou seja: } \quad \hat{d}=\left(\begin{array}{c}
0 \\
-2
\end{array}\right)
\end{aligned}
$$

O tamanho do passo $\alpha$ nessa direção de descida factível é tomado como o menor valor encontrado entre o máximo valor de $\alpha$ para não se sair da região de factibilidade $(\bar{\alpha})$ e a resolução do problema de minimização unidimensional (através da 
Regra de Armijo ou das Buscas Exatas). No caso do exemplo, o menor dos dois valores é:

$$
0 \leq x_{2}+\alpha d_{2} \leq 2 \Rightarrow 0 \leq 1+\alpha(-2) \leq 2 \Rightarrow-1 \leq-2 \alpha \leq 1 \Rightarrow \alpha \leq 0,5
$$

Portanto, o maior valor de $\alpha$ possível é dado por: $\alpha=0,5$

A nova solução será dada por:

$$
\hat{x}=\left(\begin{array}{l}
2 \\
1
\end{array}\right)+0,5\left(\begin{array}{c}
0 \\
-2
\end{array}\right)=\left(\begin{array}{l}
2 \\
0
\end{array}\right)
$$

Agora, $d=-\nabla f(\hat{x})=\left(\begin{array}{ll}2 & 0\end{array}\right)$

As direções factíveis em $\hat{x}$ devem satisfazer $d_{1} \leq 0 \quad$ e $\quad d_{2} \geq 0$.

Deseja-se que:

$$
\frac{\partial f(\hat{x})}{\partial x_{1}} d_{1}+\frac{\partial f(\hat{x})}{\partial x_{2}} d_{2}<0, \text { ou seja: } \quad-2 d_{1}+0 d_{2}<0 \text {. }
$$

Note que esta inequação não pode ser verificada para qualquer que seja a direção factível. Neste caso $\hat{\mathrm{d}}=0$.

Assim as condições de $1^{\underline{a}}$ ordem enunciadas nos teoremas anteriores são verificadas. Como a função é convexa, então $x^{*}=\left(\begin{array}{l}2 \\ 0\end{array}\right)$ é solução ótima.

\section{3 - Método do Gradiente com variáveis canalizadas}

O Método do Gradiente com variáveis canalizadas utiliza a direção de descida factível apresentada no item anterior.

O algoritmo para a determinação da direção de descida factível pelo Método do Gradiente com variáveis canalizadas é apresentado a seguir:

Para $x_{i}=a_{i}$, tem-se: 
$d_{i}= \begin{cases}-\frac{\partial f(\bar{x})}{\partial x_{i}}, & \text { se } \frac{\partial f(\bar{x})}{\partial x_{i}}<0 \\ 0, \quad \text { caso } & \text { contrário }\end{cases}$

Para $x_{i}=b_{i}$, tem-se:

$d_{i}= \begin{cases}-\frac{\partial f(\bar{x})}{\partial x_{i}}, & \text { se } \frac{\partial f(\bar{x})}{\partial x_{i}}>0 \\ 0, \quad \text { caso } & \text { contrário }\end{cases}$

Para $a_{i}<x_{i}<b_{i}$, tem-se:

$d_{i}=-\frac{\partial f(\bar{x})}{\partial x_{i}}$

O valor de $\alpha$ deve ser tomado como o menor dos dois valores $\bar{\alpha} \quad e \quad \alpha_{b u s c a}$.

A rigor, a busca unidimensional para se determinar $\alpha_{\text {busca }}$ é feita considerando-se $\bar{\alpha}$ :

$$
\begin{aligned}
& \min f(\bar{x}+\alpha d) \\
& 0 \leq \alpha \leq \bar{\alpha}
\end{aligned}
$$

E portanto, $\alpha_{\text {busca }}$ fornece o passo.

\section{Exemplo:}

Resolver o problema:

$$
\begin{aligned}
& \min f(x)=\frac{1}{2} x^{T} H x \quad a \leq x \leq b \quad \text { para: } \\
& x=\left(\begin{array}{l}
x_{1} \\
x_{2}
\end{array}\right) \quad a=\left(\begin{array}{l}
a_{1} \\
a_{2}
\end{array}\right) \quad b=\left(\begin{array}{l}
b_{1} \\
b_{2}
\end{array}\right) \quad H=\left[\begin{array}{ll}
h_{11} & h_{12} \\
h_{21} & h_{22}
\end{array}\right],
\end{aligned}
$$


onde a matriz $H$ é quadrada, simétrica e definida positiva.

$$
\begin{aligned}
& \nabla f=H x \\
& \nabla^{2} f=H
\end{aligned}
$$

\section{Afirmação:}

$x^{T} H x=1$ é a equação de uma elipse, com eixos nas direções dos auto-vetores e comprimentos $\frac{2}{\sqrt{\lambda_{1}}}, \frac{2}{\sqrt{\lambda_{2}}}$ respectivamente.

\section{Prova:}

Considere-se inicialmente um teorema da álgebra linear:

Se $H$ é simétrica e definida positiva, então tem uma base ortonormal de auto-vetores e os auto-valores são positivos. Isto é:

$$
H u_{i}=\lambda_{i} u_{i}
$$

com $\lambda_{\mathrm{i}}>0$ : auto-valor

$\mathrm{u}_{\mathrm{i}}$ : auto-vetor

$$
\left\{\begin{array}{l}
u_{1}^{T} \cdot u_{2}=0 \\
\left\|u_{i}\right\|=1
\end{array}\right.
$$

Sejam: $Q=\left[\begin{array}{ll}u_{1} & u_{2}\end{array}\right] \quad$ matriz dos auto-vetores

$$
\Lambda=\left[\begin{array}{cc}
\lambda_{1} & 0 \\
0 & \lambda_{2}
\end{array}\right] \quad \text { matriz diagonal dos auto-valores }
$$

Pode-se escrever:

$$
H\left[\begin{array}{ll}
u_{1} & u_{2}
\end{array}\right]=\left[\begin{array}{ll}
u_{1} & u_{2}
\end{array}\right]\left[\begin{array}{cc}
\lambda_{1} & 0 \\
0 & \lambda_{2}
\end{array}\right] \quad \text { ou } \quad H Q=Q \Lambda
$$


Portanto, $H=Q \Lambda Q^{T}$.

Assim, $x^{T} H x=1 \Leftrightarrow \underbrace{x^{T} Q}_{y^{T}} \Lambda \underbrace{Q^{T} x}_{y}=1$

Definindo $y=Q^{T} x$ (o que equivale à mudança de variáveis: $x=Q y$, onde $y$ é o vetor de coordenadas na base formada pelas colunas da matriz $P$, isto é, os auto-vetores de $H$ ), obtêm-se:

$$
\begin{aligned}
& \Rightarrow\left(\begin{array}{ll}
y_{1} & y_{2}
\end{array}\right)\left[\begin{array}{cc}
\lambda_{1} & 0 \\
0 & \lambda_{2}
\end{array}\right]\left(\begin{array}{l}
y_{1} \\
y_{2}
\end{array}\right)=1 \\
& \Rightarrow \lambda_{1}\left(y_{1}\right)^{2}+\lambda_{2}\left(y_{2}\right)^{2}=1 \\
& \Rightarrow\left(\frac{y_{1}}{\sqrt{\lambda_{1}^{-1}}}\right)^{2}+\left(\frac{y_{2}}{\sqrt{\lambda_{2}^{-1}}}\right)^{2}=1
\end{aligned}
$$

Considere-se da geometria analítica que a equação reduzida da elipse de centro na origem e focos no eixo $\boldsymbol{O} \boldsymbol{x}$ é:

$$
\left(\frac{x}{a}\right)^{2}+\left(\frac{y}{b}\right)^{2}=1,
$$

e os comprimentos dos eixos são $2 a$ e $2 b$. Isto mostra a afirmação. A figura (3.6) ilustra a elipse nas variáveis $x$ e $y$. Note-se que nas variáveis $y$ ela está em sua forma reduzida.

Daí, pode-se concluir que:

$$
\left\{\begin{array}{l}
\lambda_{1}=\frac{1}{a^{2}} \\
\lambda_{2}=\frac{1}{b^{2}}
\end{array}\right.
$$




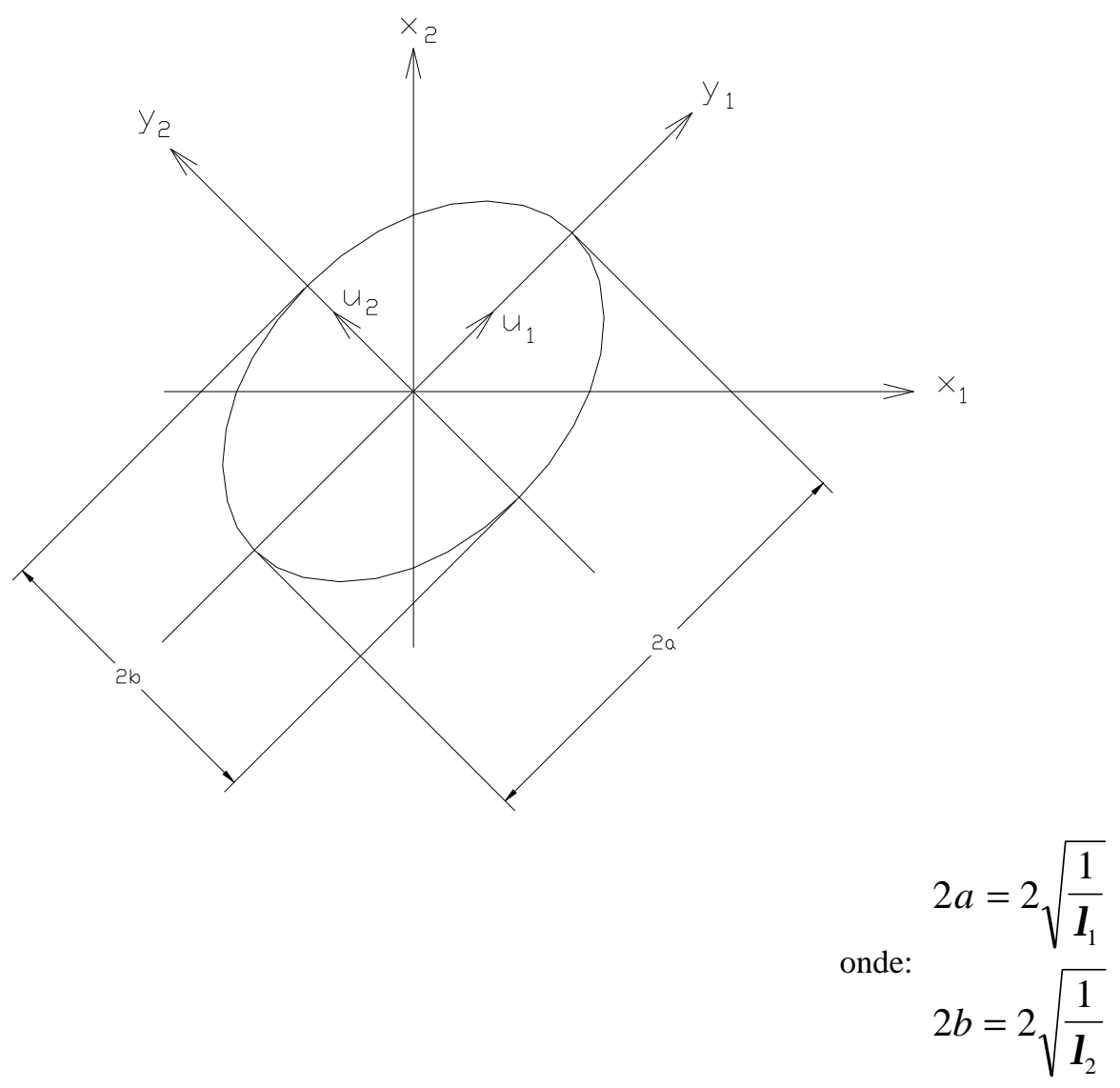

FIGURA 3.6 - Comprimentos e direções dos eixos de uma elipse

\section{Exemplo:}

Apenas para explicar com um problema numérico, coloca-se inicialmente o problema inverso, isto é, dados os auto-valores e auto-vetores, determinar a matriz $\mathrm{He}$ a partir dela e da região de factibilidade encontre a solução ótima para o problema de otimização:

No plano, através de uma rotação de eixos, a matriz dos auto-vetores é dada por:

$$
Q=\left[\begin{array}{cc}
\cos \theta & -\operatorname{sen} \theta \\
\operatorname{sen} \theta & \cos \theta
\end{array}\right]
$$

Resolvendo o problema enunciado acima para os seguintes dados:

Rotação de eixos de $45^{\circ}: \quad Q=\left[\begin{array}{cc}\frac{1}{\sqrt{2}} & -\frac{1}{\sqrt{2}} \\ \frac{1}{\sqrt{2}} & \frac{1}{\sqrt{2}}\end{array}\right]$ 
auto-valores: $\lambda_{1}=1, \quad \lambda_{2}=10 \Rightarrow \Lambda=\left[\begin{array}{cc}1 & 0 \\ 0 & 10\end{array}\right]$

intervalo: $\quad a=\left(\begin{array}{l}1 \\ 0\end{array}\right) \quad b=\left(\begin{array}{l}5 \\ 5\end{array}\right)$

Então,

$H=Q \Lambda Q^{T}=\left[\begin{array}{cc}\frac{1}{\sqrt{2}} & -\frac{1}{\sqrt{2}} \\ \frac{1}{\sqrt{2}} & \frac{1}{\sqrt{2}}\end{array}\right]\left[\begin{array}{cc}1 & 0 \\ 0 & 10\end{array}\right]\left[\begin{array}{cc}\frac{1}{\sqrt{2}} & \frac{1}{\sqrt{2}} \\ -\frac{1}{\sqrt{2}} & \frac{1}{\sqrt{2}}\end{array}\right]$
$H=\left[\begin{array}{cc}5,5 & -4,5 \\ -4,5 & 5,5\end{array}\right]$

Assim, esta matriz tem os auto-valores 1 e 10, e os auto-vetores na matriz $Q$.

Considere-se agora o problema:

$\min f(x)=\frac{1}{2} x^{T} H x$, sujeito à: $1 \leq \mathrm{x}_{1} \leq 5, \quad 0 \leq \mathrm{x}_{2} \leq 5$

Explicitando-se $f(x)$, obtêm-se:

$$
\begin{aligned}
& f(x)=\frac{1}{2}\left(\begin{array}{ll}
x_{1} & x_{2}
\end{array}\right)\left[\begin{array}{cc}
5,5 & -4,5 \\
-4,5 & 5,5
\end{array}\right]\left(\begin{array}{l}
x_{1} \\
x_{2}
\end{array}\right) \\
& f(x)=2,75 x_{1}^{2}-4,5 x_{1} x_{2}+2,75 x_{2}^{2}
\end{aligned}
$$

As curvas de nível de $f(x)$ estão representadas na figura (3.7).

Cálculo da solução exata: 


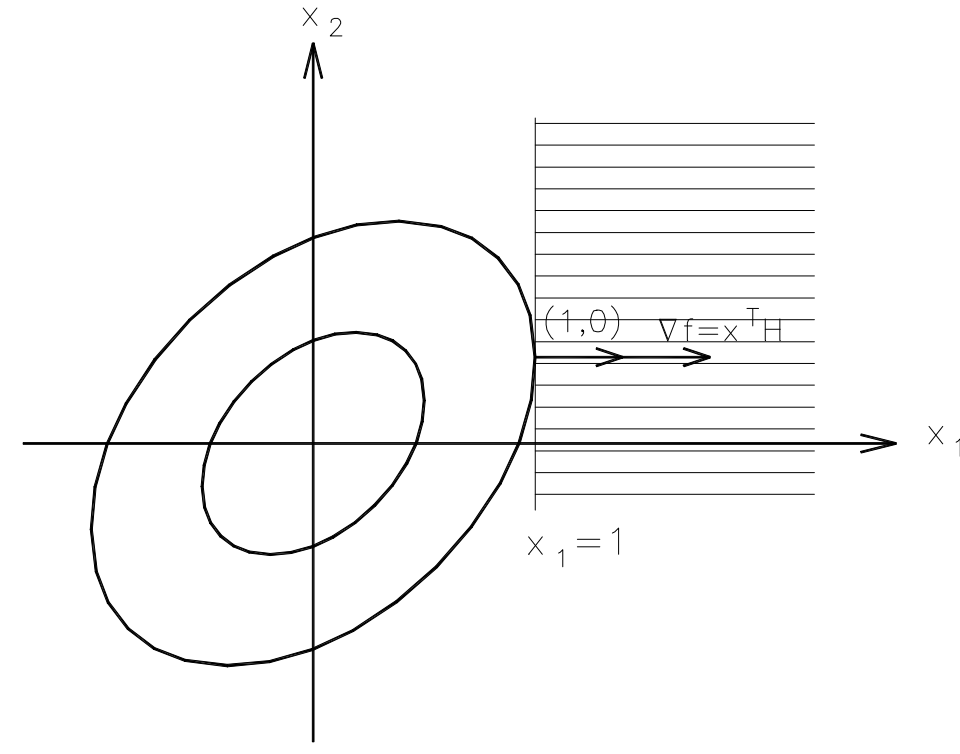

FIGURA 3.7 - Solução ótima

Note-se na figura (3.7), que a solução ótima no ponto onde a curva de nível de $f$ (de valor desconhecido) tangencia a reta: $x_{1}=1$. Como $\nabla f$ é perpendicular à curva de nível pode-se escrever (neste caso $\nabla f=x^{T} H$ ):

$$
\begin{aligned}
& \left\{\begin{array}{l}
x^{T} H+\eta(1 \quad 0)=0 \\
x_{1}=1
\end{array},\right. \\
& \left\{\begin{array}{l}
\left(\begin{array}{ll}
x_{1} & x_{2}
\end{array}\right)\left[\begin{array}{cc}
5,5 & -4,5 \\
-4,5 & 5,5
\end{array}\right]+\eta\left(\begin{array}{l}
1 \\
0
\end{array}\right)=\left(\begin{array}{l}
0 \\
0
\end{array}\right), \quad \text { ou } \\
x_{1}=1
\end{array}\right. \\
& 5,5 x_{1}-4,5 x_{2}+\eta=0 \\
& -4,5 x_{1}+5,5 x_{2}=0 \\
& x_{1}=1 \\
& \left\{\begin{array}{l}
x_{1}=1 \\
x_{2}=0,818181 \\
\eta=-1,818181
\end{array}\right.
\end{aligned}
$$


Cálculo da solução através do Método do Gradiente para variáveis canalizadas:

Solução inicial aproximada: $x^{0}=\left(\begin{array}{l}5 \\ 5\end{array}\right)$

\section{1a iteração:}

gradiente: $\quad \nabla f\left(x^{0}\right)=\left(\begin{array}{ll}5 & 5\end{array}\right)\left[\begin{array}{cc}5,5 & -4,5 \\ -4,5 & 5,5\end{array}\right]=\left(\begin{array}{l}5 \\ 5\end{array}\right)$

direção: $\quad d^{1}=-\nabla f\left(x^{0}\right)=\left(\begin{array}{l}-5 \\ -5\end{array}\right)$

busca:

$$
\begin{aligned}
& \left.\begin{array}{l}
\bar{\alpha}_{1}=\frac{1-5}{-5}=0,8 \\
\bar{\alpha}_{2}=\frac{0-5}{-5}=1,0
\end{array}\right\} \rightarrow \therefore \bar{\alpha}=0,8 \\
& \alpha_{\text {busca }}=\frac{-\left(\begin{array}{ll}
5 & 5
\end{array}\right)\left[\begin{array}{cc}
5,5 & -4,5 \\
-4,5 & 5,5
\end{array}\right]\left(\begin{array}{l}
-5 \\
-5
\end{array}\right)}{\left(\begin{array}{ll}
-5 & -5
\end{array}\right)\left[\begin{array}{cc}
5,5 & -4,5 \\
-4,5 & 5,5
\end{array}\right]\left(\begin{array}{l}
-5 \\
-5
\end{array}\right)}=\frac{50}{50}=1,0
\end{aligned}
$$

Deve-se tomar o menor valor de $\alpha$ para que a nova solução seja factível, portanto, $\alpha=0,8$.

$$
\text { nova solução: } x^{1}=\left(\begin{array}{l}
5 \\
5
\end{array}\right)+0,8\left(\begin{array}{l}
-5 \\
-5
\end{array}\right)=\left(\begin{array}{l}
1 \\
1
\end{array}\right)
$$

\section{2a iteração:}

gradiente: $\quad \nabla f\left(x^{1}\right)=\left(\begin{array}{ll}1 & 1\end{array}\right)\left[\begin{array}{cc}5,5 & -4,5 \\ -4,5 & 5,5\end{array}\right]=\left(\begin{array}{l}1 \\ 1\end{array}\right)$ 
direção: $\quad d^{2}=-\nabla f\left(x^{1}\right)=\left(\begin{array}{l}-1 \\ -1\end{array}\right)$

como $\quad x_{1}=a_{1} \quad e \quad \frac{\partial f\left(x^{1}\right)}{\partial x_{1}}>0 \Rightarrow d_{1}=0$

portanto: $\quad d^{2}=\left(\begin{array}{c}0 \\ -1\end{array}\right)$

busca:

$\forall \bar{\alpha}_{1}$

$\bar{\alpha}=\bar{\alpha}_{2}=\frac{0-1}{-1}=1$

$\alpha_{\text {busca }}=\frac{-\left(\begin{array}{ll}1 & 1\end{array}\right)\left[\begin{array}{cc}5,5 & -4,5 \\ -4,5 & 5,5\end{array}\right]\left(\begin{array}{c}0 \\ -1\end{array}\right)}{\left(\begin{array}{ll}0 & -1\end{array}\right)\left[\begin{array}{cc}5,5 & -4,5 \\ -4,5 & 5,5\end{array}\right]\left(\begin{array}{c}0 \\ -1\end{array}\right)}=\frac{1}{5,5}=0,181818$

portanto: $\alpha=0,181818$

nova solução: $x^{2}=\left(\begin{array}{l}1 \\ 1\end{array}\right)+0,181818\left(\begin{array}{c}0 \\ -1\end{array}\right)=\left(\begin{array}{c}1 \\ 0,818181\end{array}\right)$

\section{3a iteração:}

gradiente: $\quad \nabla f\left(x^{2}\right)=\left(\begin{array}{ll}1 & 0,818181\end{array}\right)\left[\begin{array}{cc}5,5 & -4,5 \\ -4,5 & 5,5\end{array}\right]=\left(\begin{array}{c}1,818181 \\ 0\end{array}\right)$

direção: $\quad d^{3}=-\nabla f\left(x^{2}\right)=\left(\begin{array}{c}-1,818181 \\ 0\end{array}\right)$

como $\quad x_{1}=a_{1} \quad e \frac{\partial f\left(x^{2}\right)}{\partial x_{1}}>0 \Rightarrow d_{1}=0$ 
portanto: $\quad d^{3}=\left(\begin{array}{l}0 \\ 0\end{array}\right)$

como $d^{3}=\left(\begin{array}{l}0 \\ 0\end{array}\right)$ o processo iterativo convergiu e a solução é:

$x^{*}=x^{2}=\left(\begin{array}{c}1 \\ 0,818181\end{array}\right)$, conforme havíamos previsto.

\section{4 - Método de Gauss-Seidel}

O Método de Gauss-Seidel com variáveis canalizadas pode ser empregado apenas em funções quadráticas, pois ele é baseado na resolução do sistema obtido a partir da condição que, no ponto de mínimo, o vetor gradiente é nulo, ou seja:

$$
\begin{aligned}
& \min f(x)=\frac{1}{2} x^{T} H x-S^{T} x \quad a \leq x \leq b \quad \text { onde: } \\
& x=\left(\begin{array}{c}
x_{1} \\
x_{2} \\
\vdots \\
x_{n}
\end{array}\right) \quad a=\left(\begin{array}{c}
a_{1} \\
a_{2} \\
\vdots \\
a_{n}
\end{array}\right) \quad b=\left(\begin{array}{c}
b_{1} \\
b_{2} \\
\vdots \\
b_{n}
\end{array}\right) \quad S=\left(\begin{array}{c}
s_{1} \\
s_{2} \\
\vdots \\
s_{n}
\end{array}\right) \\
& H=\left[\begin{array}{cccc}
h_{11} & h_{12} & \cdots & h_{1 n} \\
h_{21} & h_{22} & \cdots & h_{2 n} \\
\vdots & & \ddots & \\
h_{n 1} & h_{n 2} & \cdots & h_{n n}
\end{array}\right],
\end{aligned}
$$

e a matriz $H$ é quadrada, simétrica e definida positiva.

O gradiente e a hessiana para essa função quadrática são dados por:

$$
\begin{aligned}
& \nabla f=H x-S \\
& \nabla^{2} f=H
\end{aligned}
$$


Em pontos de mínimo (neste caso global), o vetor gradiente se anula, ou seja:

$$
\begin{aligned}
& \nabla f=H x-S=0 \Rightarrow H x=S, \text { ou } \\
& {\left[\begin{array}{cccc}
h_{11} & h_{12} & \cdots & h_{1 n} \\
h_{21} & h_{22} & \cdots & h_{2 n} \\
\vdots & & \ddots & \\
h_{n 1} & h_{n 2} & \cdots & h_{n n}
\end{array}\right]\left(\begin{array}{c}
x_{1} \\
x_{2} \\
\vdots \\
x_{n}
\end{array}\right)=\left(\begin{array}{c}
s_{1} \\
s_{2} \\
\vdots \\
s_{n}
\end{array}\right)}
\end{aligned}
$$

O algoritmo básico de Gauss-Seidel, para um sistema de ordem $n$, tem a seguinte expressão geral para o refinamento da solução:

$$
\begin{aligned}
& h_{i i} x_{i}^{k+1}=-\sum_{j=1}^{i-1}\left(h_{i j} x_{j}^{k+1}\right)-\sum_{j=i+1}^{n}\left(h_{i j} x_{j}^{k}\right)+s_{i}, \\
& (i=1,2,3, \ldots, n)
\end{aligned}
$$

onde:

$h_{i j}$ : elemento da i-ésima linha e j-ésima coluna da matriz hessiana;

$x_{i}^{k+1}:$ i-ésima coordenada do vetor de incógnitas para a iteração $K+1$.

A aproximação obtida após um certo número de iterações é considerada suficiente, quando for verificada a seguinte condição:

$$
\begin{aligned}
& \max \left|x_{i}^{k+1}-x_{i}^{k}\right|<\text { tol } \\
& (i=1,2, \ldots, n)
\end{aligned}
$$

O procedimento iterativo exige a adoção de uma solução inicial aproximada. A imposição de condições de contorno é bastante simples, neste caso, pois basta impor que a variável $\mathrm{x}_{\mathrm{i}}$ tenha seu valor no intervalo de definição $\left(a_{i} \leq x_{i} \leq b_{i}\right)$, obtido em todas as iterações através do uso de um operador de projeção.

Para diminuir o número total de iterações, é interessante fazer uso do procedimento de relaxação, o qual consiste, fundamentalmente, de uma ponderação entre as aproximações $K$ e $K+1$, para fins de atualização da solução $K+1$. 
A expressão geral da relaxação é a seguinte:

$$
x_{i}^{k+1}=(1-w) x_{i}^{k}+w x_{i}^{k+1}
$$

O parâmetro $w$ é limitado no intervalo aberto $(0,2)$, devendo-se avaliar o valor ideal para cada caso.

Para a obtenção da solução aproximada para a variável $\{x\}$, o Método de Gauss-Seidel com relaxação foi implementado segundo o seguinte algoritmo:

Para $k=0,1,2, \ldots$, número máximo de iterações e $w \in(0,2)$, tem-se:

$$
\begin{aligned}
& h_{i i} x_{i}^{k+1}=-\sum_{j=1}^{i-1}\left(h_{i j} x_{j}^{k+1}\right)-\sum_{j=i+1}^{n}\left(h_{i j} x_{j}^{k}\right)+s_{i} ; \\
& (i=1,2,3, \ldots, n) \\
& x_{i}^{k+1}=(1-w) x_{i}^{k}+w x_{i}^{k+1}
\end{aligned}
$$

Como a variável $x_{i}^{k+1}$ possui um limitante inferior e superior $a_{i}, b_{i}$ respectivamente, a projeção da variável sobre este intervalo é feita da seguinte forma:

$$
x_{i}^{k+1}=\min \left\{b_{i}, \operatorname{máx}\left\{a_{i}, x_{i}^{k+1}\right\}\right\}
$$

A figura (3.8) ilustra o efeito da projeção:

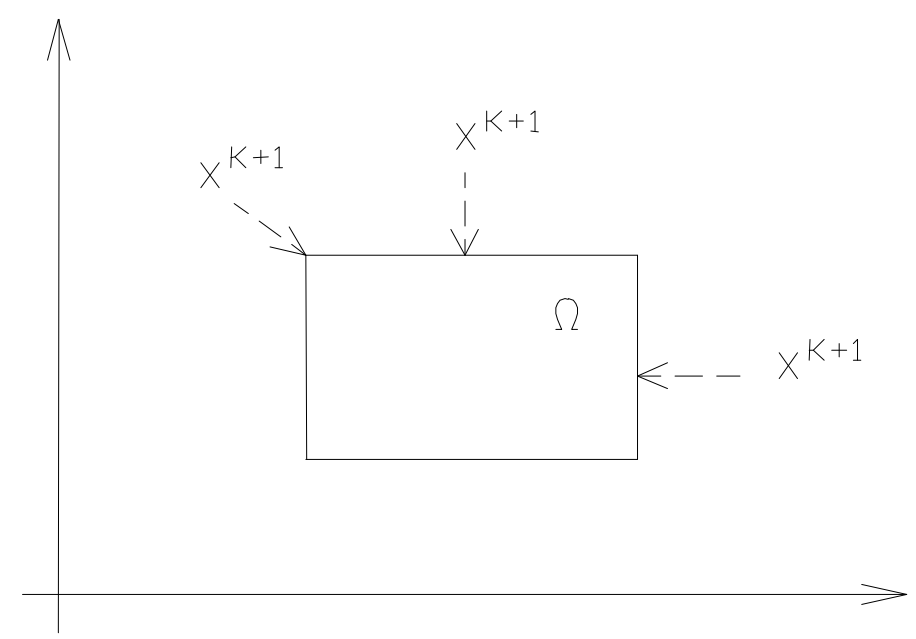

FIGURA 3.8 - A projeção em (3.28) 


\section{5 - Método de Newton e Quase-Newton combinados com a estratégia dos Conjuntos Ativos}

O Método de Newton, que consiste em desenvolver uma função $f$, por Taylor, em torno de um ponto $x^{K}$, até a $2^{\text {a }}$ ordem obtendo-se uma aproximação quadrática, pode ser combinado com a estratégia dos conjuntos ativos para resolver problemas com variáveis canalizadas. Esta estratégia induz o Método de Newton a realizar uma minimização na face sempre que uma variável, ou mais, já tenham atingido suas restrições e sendo que a direção de Newton irrestrita conduza o problema à soluções fora da região de factibilidade.

A figura (3.9) ilustra um problema tridimensional com $x^{k} \in \Omega \mathrm{e}$ $\Omega=\left\{x^{k} \in R^{3} / a \leq x^{k} \leq b\right\}$. Particularmente, o ponto $x^{K}$ pertencente a região de factibilidade $\Omega$, representada por um cubo na figura e se encontra por hipótese, numa das suas faces. Aplicando-se o método de Newton em $x^{K}$, obtém-se uma direção de descida que conduz o problema a uma nova solução $x^{*}$ fora da região de factibilidade, desrespeitando-se assim as condições de restrições impostas ao problema:

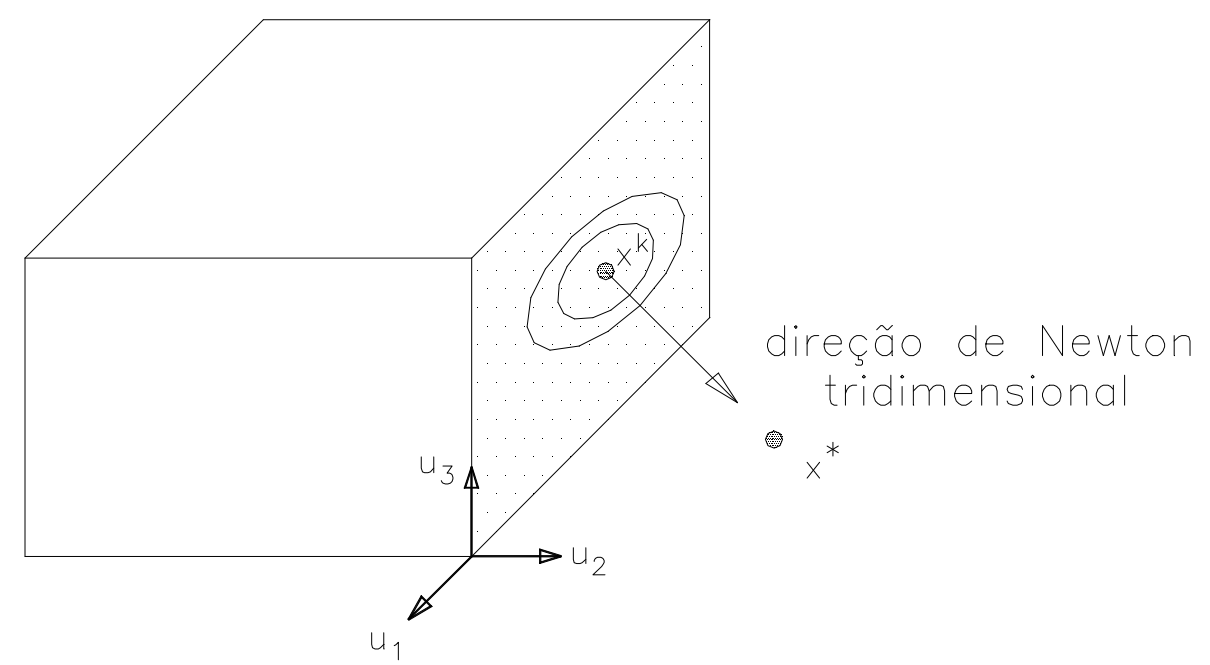

FIGURA 3.9 - Direção de Newton tridimensional

Portanto, no caso ilustrado, o método de Newton deveria ser aplicado restringindo-se a minimização à dimensão da face em que se encontra o ponto $x^{K}$, ou seja, numa região bidimensional; daí vem a necessidade de se combinar o método de 
Newton com a estratégia dos conjuntos ativos, que permite ao método realizar uma minimização na face sempre que ocorrer um caso como o ilustrado na figura (3.9). Segundo essa estratégia, as coordenadas que atingirem suas restrições tornam-se ativas (fixas), garantindo assim a procura de soluções dentro da região de factibilidade.

Qualquer ponto nessa face se escreve como:

$$
x=x^{k}+k_{1} u_{1}+k_{3} u_{3}
$$

onde $\quad x=\left(\begin{array}{l}x_{1} \\ x_{3}\end{array}\right)$

A função a ser minimizada é:

$$
f(x)=f\left(x^{k}+k_{1} u_{1}+k_{3} u_{3}\right),
$$

que depende de $k_{1}$ e $k_{3}$ ( 2 dimensões)

Particularmente, se $x^{k}$ estiver numa aresta, a nova solução será:

$$
x=x^{k}+k_{1} u_{1}
$$

onde $x=\left(x_{1}\right)$, tratando-se assim de um problema unidimensional.

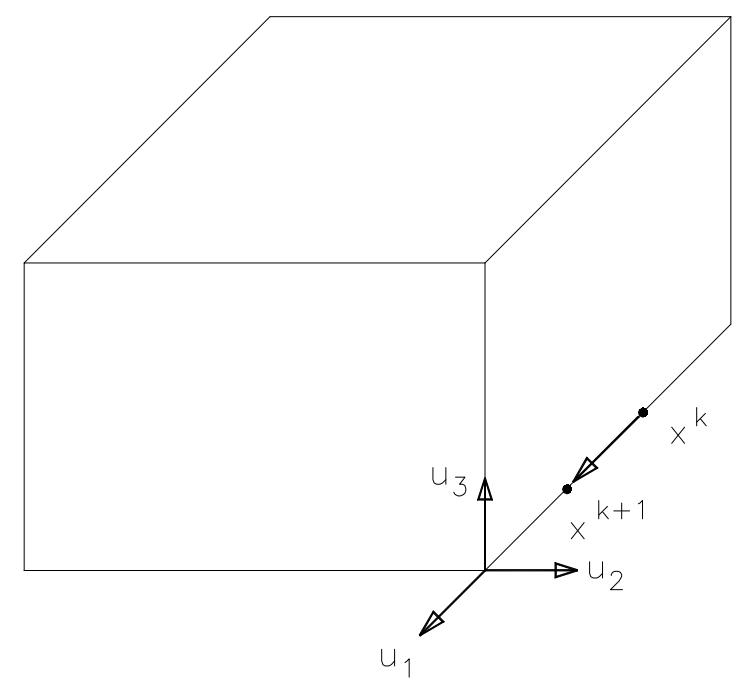


Aplicar o método de Newton na face indicada pela figura (3.9) significa desenvolver:

$$
g\left(k_{1} \quad k_{3}\right)=f\left(x^{k}+k_{1} u_{1}+k_{3} u_{3}\right),
$$

até segunda ordem (quadrática) em torno de $k_{1}=k_{3}=0$ (neste caso $x=x^{k}$ ), e determinar $k_{1}$ e $k_{3}$ que minimize a quadrática:

$$
g\left(\begin{array}{ll}
k_{1} & k_{3}
\end{array}\right)=g\left(\begin{array}{ll}
0 & 0
\end{array}\right)+\nabla g^{T}\left(\begin{array}{ll}
0 & 0
\end{array}\right)\left(\begin{array}{l}
k_{1} \\
k_{3}
\end{array}\right)+\frac{1}{2}\left(\begin{array}{ll}
k_{1} & k_{3}
\end{array}\right) \nabla^{2} g\left(\begin{array}{ll}
0 & 0
\end{array}\right)\left(\begin{array}{l}
k_{1} \\
k_{3}
\end{array}\right)
$$

O gradiente da função $g\left(k_{1} k_{3}\right)$ é dado por:

$$
\begin{aligned}
& \nabla g\left(\begin{array}{l}
k_{1} \\
k_{3}
\end{array}\right)=\nabla f\left(x^{k}+k_{1} u_{1}+k_{3} u_{3}\right) u_{1}+\nabla f\left(x^{k}+k_{1} u_{1}+k_{3} u_{3}\right) u_{3}= \\
& =\nabla f^{T}\left(x^{k}+k_{1} u_{1}+k_{3} u_{3}\right)\left[\begin{array}{ll}
u_{1} & u_{3}
\end{array}\right]=\nabla f^{T} \Phi
\end{aligned}
$$

onde $\Phi$ é a matriz formada pelas direções da face.

A segunda derivada da função $g\left(k_{1} k_{3}\right)$ é dada por:

$$
\begin{aligned}
& \nabla^{2} g\left(\begin{array}{l}
k_{1} \\
k_{3}
\end{array}\right)=u_{1}^{T} \nabla^{2} f\left(x^{k}+k_{1} u_{1}+k_{3} u_{3}\right) u_{1}+u_{3}{ }^{T} \nabla^{2} f\left(x^{k}+k_{1} u_{1}+k_{3} u_{3}\right) u_{3}= \\
& =\left[\begin{array}{ll}
u_{1} & u_{3}
\end{array}\right]^{T} \nabla^{2} f\left(x^{k}+k_{1} u_{1}+k_{3} u_{3}\right)\left[\begin{array}{ll}
u_{1} & u_{3}
\end{array}\right]=\Phi^{T} \nabla^{2} f \Phi
\end{aligned}
$$

Para a face do exemplo, a matriz $\Phi$ representa as direções $u_{1}$ e $u_{3}$, isto é:

$$
\Phi=\left[\begin{array}{ll}
1 & 0 \\
0 & 0 \\
0 & 1
\end{array}\right]
$$

O gradiente da função $g\left(k_{1} \quad k_{3}\right)$ seria: 


$$
\nabla g=\nabla f^{T} \Phi=\left(\begin{array}{lll}
\frac{\partial f}{\partial x_{1}} & \frac{\partial f}{\partial x_{2}} & \frac{\partial f}{\partial x_{3}}
\end{array}\right)\left[\begin{array}{ll}
1 & 0 \\
0 & 0 \\
0 & 1
\end{array}\right]=\left(\begin{array}{ll}
\frac{\partial f}{\partial x_{1}} & \frac{\partial f}{\partial x_{3}}
\end{array}\right)
$$

e sua segunda derivada seria dada por:

$$
\begin{aligned}
& \nabla^{2} g=\Phi^{T} \nabla^{2} f \Phi=\left[\begin{array}{lll}
1 & 0 & 0 \\
0 & 0 & 1
\end{array}\right]\left[\begin{array}{lll}
\frac{\partial^{2} f}{\partial x_{1}^{2}} & \frac{\partial^{2} f}{\partial x_{1} x_{2}} & \frac{\partial^{2} f}{\partial x_{1} x_{3}} \\
\frac{\partial^{2} f}{\partial x_{1} x_{2}} & \frac{\partial^{2} f}{\partial x_{2}^{2}} & \frac{\partial^{2} f}{\partial x_{2} x_{3}} \\
\frac{\partial^{2} f}{\partial x_{1} x_{3}} & \frac{\partial^{2} f}{\partial x_{2} x_{3}} & \frac{\partial^{2} f}{\partial x_{3}^{2}}
\end{array}\right]\left[\begin{array}{ll}
1 & 0 \\
0 & 0 \\
0 & 1
\end{array}\right]= \\
& =\left[\begin{array}{ll}
\frac{\partial^{2} f}{\partial x_{1}^{2}} & \frac{\partial^{2} f}{\partial x_{1} x_{3}} \\
\frac{\partial^{2} f}{\partial x_{1} x_{3}} & \frac{\partial^{2} f}{\partial x_{3}^{2}}
\end{array}\right]
\end{aligned}
$$

Substituindo-se as expressões (3.34) e (3.35) em (3.33), tem-se:

$$
g\left(\begin{array}{ll}
k_{1} & k_{3}
\end{array}\right)=f\left(x^{k}\right)+\nabla f^{T}\left(x^{k}\right) \Phi\left(\begin{array}{l}
k_{1} \\
k_{3}
\end{array}\right)+\frac{1}{2}\left(\begin{array}{ll}
k_{1} & k_{3}
\end{array}\right) \Phi^{T} \nabla^{2} f\left(x^{k}\right) \Phi\left(\begin{array}{l}
k_{1} \\
k_{3}
\end{array}\right)
$$

O ponto de mínimo de $g\left(k_{1} k_{3}\right)$ é dado por:

$$
\nabla f^{T}\left(x^{k}\right) \Phi+\left(k_{1} \quad k_{3}\right) \Phi^{T} \nabla^{2} f\left(x^{k}\right) \Phi=0
$$

ou seja, o cálculo da direção de descida no método de Newton combinado com a teoria dos conjuntos ativos, pode ser determinado através da resolução do sistema linear proposto:

$$
\Phi^{T} \nabla^{2} f\left(x^{k}\right) \Phi\left(\begin{array}{l}
k_{1} \\
k_{3}
\end{array}\right)=-\nabla f^{T}\left(x^{k}\right) \Phi
$$

onde o primeiro termo da expressão representa a matriz hessiana original, em $x^{k}$, considerando apenas as coordenadas da face; e o segundo termo representa o vetor gradiente original, em $x^{k}$, considerando apenas as coordenadas da face. 
A nova solução para o problema de minimização descrito em (3.30) é calculada pela expressão:

$$
x^{k+1}=x^{k}+k_{1} u_{1}+k_{3} u_{3}=x^{k}+\Phi\left(\begin{array}{l}
k_{1} \\
k_{3}
\end{array}\right)
$$

Note que, quando a solução $x^{k}$ estiver no interior de uma região de factibilidade, ou seja, nenhuma coordenada do ponto $x^{k}$ atingiu sua restrição, a matriz $\Phi$ formada pelas direções da face será a própria matriz identidade. Portanto, a direção de descida calculada através da resolução do sistema linear proposto em (3.38) passa a ser a mesma descrita pelo método de Newton para problemas irrestritos.

Generalizando-se para o espaço $\mathrm{R}^{\mathrm{n}}$, teriamos:

$$
x^{k+1}=x^{k}+k_{1} u_{1}+k_{2} u_{2}+\cdots+k_{n} u_{n}=x^{k}+\Phi\left(\begin{array}{c}
k_{1} \\
k_{2} \\
\vdots \\
k_{n}
\end{array}\right)=x^{k}+\Phi d^{k}
$$

onde $x^{k}=\left(\begin{array}{c}x_{1} \\ x_{2} \\ \vdots \\ x_{n}\end{array}\right)$ é o vetor solução, $d^{k}=\left(\begin{array}{c}k_{1} \\ k_{2} \\ \vdots \\ k_{n}\end{array}\right)$ é o vetor de descida na iteração k e $\Phi$ é a matriz formada pelas direções da face.

Portanto, a expressão (3.38) seria:

$$
\left\lfloor\Phi^{T} \nabla^{2} f\left(x^{k}\right) \Phi\right\rfloor d^{k}=-\nabla f^{T}\left(x^{k}\right) \Phi
$$

Isso define o método de Newton combinado com a estratégia dos conjuntos ativos. Note que a expressão (3.41) tem a mesma formulação daquela descrita em (2.39) pelo método de Newton irrestrito, considerando-se também a minimização na face através da matriz de direções $\Phi$. Dessa forma, o método de Newton pode ser estendido à problemas com restrições nas variáveis de interesse. 
Os métodos do tipo Quase-Newton também podem ser combinados com a estratégia dos conjuntos ativos, para que ele possa promover uma minimização na face quando necessário, naturalmente, para o caso de problemas com restrições. Seu algoritmo básico para a regra de aproximação da Hessiana proposta por DavidonFletcher-Powell, consideraria a influência das coordenadas ativas através da matriz das direções da face $\Phi$, ou seja:

Passo inicial) escolha $Y_{0}$ simétrica positiva definida e $x^{0}$ um ponto qualquer;

Passo 1) Minimização na face: $Y r_{K}=\Phi^{T} Y_{k} \Phi$

Passo 2) $d r^{K}=-Y r_{K} q r_{K}$, onde $q r_{K}=\Phi^{T} \nabla f\left(x^{K}\right)$;

Passo 3) $x^{K+1}=x^{K}+\alpha_{K} d^{K}$, onde $d r^{k}=\Phi^{T} d^{k}$. Sendo $\alpha_{K}$ tal que minimize $f\left(x^{K}+\alpha_{K} d^{K}\right)$

$$
p r_{K}=\alpha_{K} d r^{K} \text { e } \quad q r_{K+1}=\Phi^{T} \nabla f\left(x^{K+1}\right) ;
$$

Passo 4) $q r_{K}=q r_{K+1}-q r_{K}$

$$
Y r_{K+1}=Y r_{K}+\frac{p r_{K} p r_{K}^{T}}{p r_{K}^{T} q r_{K}}-\frac{Y r_{K} q r_{K} q r_{K}^{T} Y r_{K}}{q r_{K}^{T} Y r_{K} q r_{K}} ;
$$

$K=K+1$ e volte para o passo 1 


\section{CAPÍTULO 4 - MODELAGEM DE ESTRUTURAS}

$\mathrm{Na}$ análise de estruturas em regime elástico, a formulação do método pressupõe que existem apenas duas manifestações de energia mais relevantes. A primeira é conhecida como a energia potencial das cargas, que está relacionada com o trabalho das forças atuantes, ou forças externas; a segunda, é conhecida como a energia de deformação, que está relacionada com o trabalho das forças internas.

O Princípio da Conservação da Energia pressupõe que, em qualquer situação, a energia retirada de uma das manifestações passa a pertencer à outra. Por exemplo, o trabalho produzido pelas cargas atuantes segundo os deslocamentos da estrutura acumula-se sob a forma de energia de deformação da estrutura.

Quando o trabalho da carga for positivo, entende-se que a carga perdeu potencial, ocorrendo assim uma diminuição na capacidade de trabalho da carga. Dessa forma, simbolizando-se por $\Psi$ o potencial da carga, e por $\mathrm{T}$ de trabalho da carga, temse:

$$
\partial \Psi=-\partial T
$$

onde o símbolo $\partial$ representa variação.

Essa relação entre o trabalho da carga e sua energia potencial tem como explicação física a existência de um campo de força, como, por exemplo, o gravitacional. $\mathrm{O}$ trabalho positivo da carga corresponde, no fundo, a uma queda nesse campo.

A energia de deformação origina-se do trabalho das tensões segundo as deformações decorrentes dos deslocamentos sofridos pela estrutura. Naturalmente, o trabalho das forças internas não se realiza em movimentos de corpo rígido, pois não há deformação. Assim, a energia em consideração pode ser representada por: 


$$
U=\frac{1}{2} \int_{V} \sigma^{T} \varepsilon d V
$$

onde: V é o volume da estrutura

$\sigma$ é um vetor contendo as componentes de tensão

$\varepsilon$ é um vetor contendo as componentes de deformação.

O princípio da Conservação da Energia, tendo-se em vista apenas as duas manifestações de energia consideradas, permite escrever:

$$
\pi=U+\Psi=\text { cte }
$$

onde $\pi$ representa a chamada energia potencial total. Consequentemente a variação de energia total é nula:

$$
\partial \pi=\partial U+\partial \Psi=0,
$$

ou seja, no fenômeno de deformação da estrutura pela ação de cargas a energia potencial total não se altera; o que ganha energia uma dada manifestação decorre da diminuição da outra.

Assim, o princípio da Conservação da Energia impõe condição estacionária para a energia total. Essa condição serve de suporte para a obtenção de soluções exatas ou aproximadas, por exemplo, para os deslocamentos em estruturas. Todavia, pretende-se neste trabalho abordar apenas o procedimento que conduz a soluções aproximadas.

Um teorema complementar muito importante neste estudo afirma que nas estruturas com regime elástico e com pequenas deformações à situação em equilíbrio corresponde a um mínimo da função da energia potencial total.

Uma das importantes aplicações do Método da Energia é o cálculo de deslocamentos de estruturas no âmbito da análise estática.

Uma estrutura inicialmente indeformada, quando submetida a um certo carregamento, por hipótese invariável ao longo do tempo, atinge uma situação de equilíbrio em correspondência a uma nova posição deformada.

Nessa posição de equilíbrio, a estrutura apresenta deslocamentos medidos com relação à sua posição inicial, cuja a ordem de grandeza depende, entre outros fatores, do tipo de material de que é composta e da sua geometria. 
O método, como se viu, envolve duas formas de energia: a externa associada ao carregamento e a interna.

A energia externa é dada, basicamente, pelo produto da carga pelo deslocamento do seu ponto de aplicação. A energia interna ou de deformação é obtida pelo procedimento que se segue.

Em linhas gerais, a energia de deformação acumulada num elemento de volume é dada pela seguinte expressão:

$$
U=\frac{1}{2} \int_{V}\left(\sigma_{x} \varepsilon_{x}+\sigma_{y} \varepsilon_{y}+\sigma_{z} \varepsilon_{z}+\tau_{x y} \gamma_{x y}+\tau_{x z} \gamma_{x z}+\tau_{y z} \gamma_{y z}\right) d V
$$

ou seja:

$$
U=\frac{1}{2} \int_{V} \sigma^{T} \varepsilon d V
$$

Quando o material for considerado elástico linear, as deformações e as tensões podem ser relacionadas pela Lei de Hooke, ou seja:

$$
\begin{aligned}
& \varepsilon_{x}=\frac{1}{E}\left[\sigma_{x}-v\left(\sigma_{y}+\sigma_{z}\right)\right] \\
& \varepsilon_{y}=\frac{1}{E}\left[\sigma_{y}-v\left(\sigma_{x}+\sigma_{z}\right)\right] \\
& \varepsilon_{z}=\frac{1}{E}\left[\sigma_{z}-v\left(\sigma_{x}+\sigma_{y}\right)\right] \\
& \gamma_{x y}=\frac{1}{G} \tau_{x y} \\
& \gamma_{x z}=\frac{1}{G} \tau_{x z} \\
& \gamma_{y z}=\frac{1}{G} \tau_{y z} \\
& G=\frac{E}{2(1+v)}
\end{aligned}
$$

caso contrário, ou seja, tratando-se de um material que não segue a Lei de Hooke, as deformações e as tensões são relacionadas não linearmente.

Portanto, de uma forma genérica, no caso linear tem-se:

$$
\sigma=D \varepsilon
$$


onde $D$ é uma matriz que reúne os chamados módulos elásticos de rigidez.

No caso particular do Estado Plano de Tensão, as componentes de tensão de uma das faces do volume elementar $d x d y d z$ são nulas; por via de consequência o mesmo acontece na face oposta. Por exemplo, as componentes nulas podem ser $\sigma_{z}=\tau_{x z}=\tau_{y z}=0$. Nessas condições, pode-se considerar uma espessura unitária para 0 elemento.

Voltando ao caso geral, combinando-se (4.8) com (4.6), a energia de deformação assume a forma:

$$
U=\frac{1}{2} \int_{V} \varepsilon^{T} D \varepsilon d V
$$

A energia potencial de forças volumétricas é dada por:

$$
\Psi=-\int_{V} u^{T} g d V
$$

e a parcela da energia potencial associada às forças de superfície é representada por:

$$
\Psi=-\int_{\Gamma} u^{T} p d \Gamma
$$

onde:

$$
u=\left\{\begin{array}{l}
u_{x} \\
u_{y}
\end{array}\right\} \quad g=\left\{\begin{array}{l}
g_{x} \\
g_{y}
\end{array}\right\} \quad p=\left\{\begin{array}{l}
p_{x} \\
p_{y}
\end{array}\right\}
$$

Tendo em vista que as relações deslocamento-deformação são as seguintes: 


$$
\begin{aligned}
& \varepsilon_{x}=\frac{\partial u_{x}}{\partial x} \\
& \varepsilon_{y}=\frac{\partial u_{y}}{\partial y} \\
& \varepsilon_{z}=\frac{\partial u_{z}}{\partial z} \\
& \gamma_{x y}=\frac{\partial u_{x}}{\partial y}+\frac{\partial u_{y}}{\partial x} \\
& \gamma_{x z}=\frac{\partial u_{x}}{\partial z}+\frac{\partial u_{z}}{\partial x} \\
& \gamma_{y z}=\frac{\partial u_{y}}{\partial z}+\frac{\partial u_{z}}{\partial y}
\end{aligned}
$$

Para o Estado Plano de Tensão, tem-se:

$$
\begin{aligned}
\varepsilon_{x} & =\frac{\partial u_{x}}{\partial x} \\
\varepsilon_{y} & =\frac{\partial u_{y}}{\partial y} \\
\gamma_{x y} & =\frac{\partial u_{x}}{\partial y}+\frac{\partial u_{y}}{\partial x}
\end{aligned}
$$

Uma formulação geral dos Elementos Finitos parte do princípio de estabelecer funções aproximadoras para os deslocamentos incógnitos de uma estrutura, baseando-se em interpolações expressas em função de deslocamentos nodais. Os deslocamentos nodais são associados à nós definidos previamente na etapa de discretização da estrutura. Nessas condições, o campo de deslocamentos fica expresso na forma:

$$
u=\phi u^{n},
$$

onde:

$\phi=$ matriz das funções de forma

$\mathrm{u}^{\mathrm{n}}=$ vetor dos deslocamentos nodais generalizados ou parâmetros nodais 
A partir do conjunto de nós, define-se uma rede de elementos e a técnica dos elementos finitos propõe, ainda, que a função aproximadora resulte da combinação das funções aproximadoras de cada elemento.

As funções aproximadoras de cada elemento têm características particulares, e entre elas destacam-se: são polinômios de grau $n$ e possuem "suporte compacto" isto é, são definidas somente no domínio do elemento assumindo valor nulo fora dele.

Logo, conclui-se que o grau das funções aproximadoras que podem ser empregadas em cada elemento depende do número de parâmetros nodais previamente definido.

Através das relações deslocamento-deformação, pode-se calcular a aproximação para as deformações. No caso plano de tensão, por exemplo:

$$
\varepsilon=\left\{\begin{array}{l}
\varepsilon_{x} \\
\varepsilon_{y} \\
\gamma_{x y}
\end{array}\right\}=\left[\begin{array}{cc}
\frac{\partial}{\partial x} & 0 \\
0 & \frac{\partial}{\partial y} \\
\frac{\partial}{\partial y} & \frac{\partial}{\partial x}
\end{array}\right]\left\{\begin{array}{l}
u_{x} \\
u_{y}
\end{array}\right\}=\left[\begin{array}{cc}
\frac{\partial}{\partial x} & 0 \\
0 & \frac{\partial}{\partial y} \\
\frac{\partial}{\partial y} & \frac{\partial}{\partial x}
\end{array}\right] \phi u^{n}=B u^{n}
$$

onde:

$$
B=\left[\begin{array}{cc}
\frac{\partial}{\partial x} & 0 \\
0 & \frac{\partial}{\partial y} \\
\frac{\partial}{\partial y} & \frac{\partial}{\partial x}
\end{array}\right] \phi, \text { que reúne as derivadas das funções de forma. }
$$

Conhecendo-se a aproximação global para o campo dos deslocamentos, o vetor de deformações $\varepsilon$ e a matriz $D$, que é função das características do material, pode-se calcular a energia de deformação, em forma aproximada para toda a estrutura:

$$
\begin{aligned}
& U_{a}=\frac{1}{2} \int_{V} \varepsilon^{T} D \varepsilon d V \\
& U_{a}=\frac{1}{2} \int_{V} u^{n^{T}} B^{T} D B u^{n} d V \\
& U_{a}=\frac{1}{2} u^{n^{T}}\left[\int_{V} B^{T} D B d V\right] u^{n}
\end{aligned}
$$


A parcela da energia potencial externa associada às forças de superfície pode ser calculada admitindo-se uma função aproximadora para essas forças:

$$
\begin{aligned}
& p=\phi_{p} p^{n} \\
& \Psi_{a}=-\int_{\Gamma} u^{T} p d \Gamma \\
& \Psi_{a}=-\int_{\Gamma} u^{n^{T}} \phi^{T} \phi_{p} p^{n} d \Gamma \\
& \Psi_{a}=-u^{n^{T}}\left[\int_{\Gamma} \phi^{T} \phi_{p} d \Gamma\right] p^{n}
\end{aligned}
$$

onde $\phi_{p}$ é a matriz das funções de forma e $p^{n}$ o vetor de forças nodais.

A energia potencial total em forma aproximada é dada pela soma algébrica das formas aproximadas da energia de deformação e da energia potencial das cargas externas, conforme expressão (4.3):

$$
\pi_{a}=U_{a}+\Psi_{a}
$$

O princípio da mínima energia potencial total aplicado como condição para a determinação dos deslocamentos nodais incógnitos da estrutura discretizada, ou seja:

$$
\min \pi_{a}=\frac{1}{2} u^{n^{T}}\left[\int_{V} B^{T} D B d V\right] u^{n}-u^{n^{T}}\left[\int_{\Gamma} \phi^{T} \phi_{p} d \Gamma\right] p^{n}
$$

$\mathrm{Na}$ expressão anterior os termos em colchetes representam a matriz de rigidez global da estrutura e o vetor de forças nodais equivalentes.

Definindo-se a matriz de rigidez e o vetor de forças nodais de um elemento por:

$$
\begin{aligned}
& K_{e}=\int_{V_{e}} B^{T} D B d V_{e} \\
& F_{e}=\left[\int_{\Gamma_{e}} \phi^{T} \phi_{p} d \Gamma_{e}\right] p^{n}
\end{aligned}
$$


A matriz de rigidez da estrutura $K$ e o vetor de forças nodais equivalentes $F$ são obtidos considerando-se a contribuição de cada elemento que compõe a estrutura.

Portanto, o funcional a ser minimizado passa a ser do tipo:

$$
\min \pi=\frac{1}{2} u^{n^{T}} K u^{n}-u^{n^{T}} F
$$

ou seja, determinar os deslocamentos nodais de uma estrutura significa resolver um problema de otimização de uma função quadrática. Daí a aplicação direta que se pode dar à programação matemática para a análise de estruturas.

Tradicionalmente os programas de elementos finitos abordam o problema de otimização irrestrita, isto é, os deslocamentos da estrutura não estão sujeitos a restrições advindas de condições particulares de vinculação como por exemplo o caso de problemas de contato unilateral. Dessa forma, os deslocamentos são calculados resolvendo-se o seguinte sistema linear:

$$
\begin{aligned}
& \partial \pi=\partial u^{n^{T}}\left\{K u^{n}-F\right\}=0 \quad \text { p/ } \quad \forall \quad u^{n} \in R^{n} \\
& \text { ou } \quad K u^{n}=F
\end{aligned}
$$

Uma vez determinados os valores nodais globais dos deslocamentos, os deslocamentos associados aos nós de cada elemento podem ser obtidos a partir de uma identificação direta.

As deformações e as tensões são finalmente calculadas por:

$$
\begin{aligned}
& \varepsilon=B u^{n} \\
& \sigma=D \varepsilon
\end{aligned}
$$

Por sua vez, no caso de análise de estruturas com restrições a deslocamentos, como por exemplo aqueles oriundos de condições particulares de vinculações, a maneira matemática mais consistente de se tratar o problema é promover uma minimização do funcional da energia total, através de métodos de otimização com variáveis canalizadas. 
Sob o ponto de vista de programação matemática, o problema de análise estrutural pode ser colocado como uma minimização de uma função quadrática sujeita ou não a restrições em suas variáveis.

A minimização de uma função quadrática é um problema do seguinte tipo:

$$
\min f(x)=\frac{1}{2} x^{n^{T}} H x^{n}+x^{n^{T}} S+C
$$

onde: $H=\nabla^{2} f(x)$ é a matriz hessiana da função $f(x)$

$$
S=\left.\nabla f(x)\right|_{x=0} \text { é o vetor gradiente da função } f(x)
$$

Como o funcional da energia total pode ser representado na forma matricial pela equação (4.21), ou seja, $\pi=\frac{1}{2} u^{n^{T}} K u^{n}-u^{n^{T}} F$, a matriz de rigidez da estrutura passa a ser a matriz hessiana da função quadrática, o vetor de forças nodais da estrutura passa a ser o vetor gradiente e os deslocamentos nodais as variáveis que podem apresentar restrições ou não.

Portanto, os métodos de otimização são úteis ferramentas matemáticas na análise de estruturas, particularmente àquelas sujeitas a restrições em seus deslocamentos. Os métodos abordados nesta pesquisa são o do Gradiente, Newton, Quase-Newton e o método iterativo de Gauss-Seidel, este último para fins de confronto com os resultados obtidos pelos anteriores.

\section{1 - Vigas}

Uma das importantes aplicações do Método da Energia é o cálculo de deslocamentos em vigas.

Uma estrutura inicialmente indeformada, quando submetida a um certo carregamento, por hipótese invariável ao longo do tempo, atinge uma situação de equilíbrio em correspondência a uma nova posição deformada; na posição de equilíbrio, a estrutura apresenta deslocamentos medidos com relação à posição inicial.

No caso da viga, uma hipótese frequentemente utilizada é a de que os deslocamentos são tais que as seções inicialmentes transversais ao eixo permanecem planas e ortogonais ao eixo após a deformação. Nessas condições para determinar a 
nova posição deformada é suficiente caracterizar o deslocamento e a inclinação do eixo em relação à sua posição inicial, inclinação esta determinada em cada um de seus pontos pela derivada da função deslocamento, como mostra a figura (4.1):
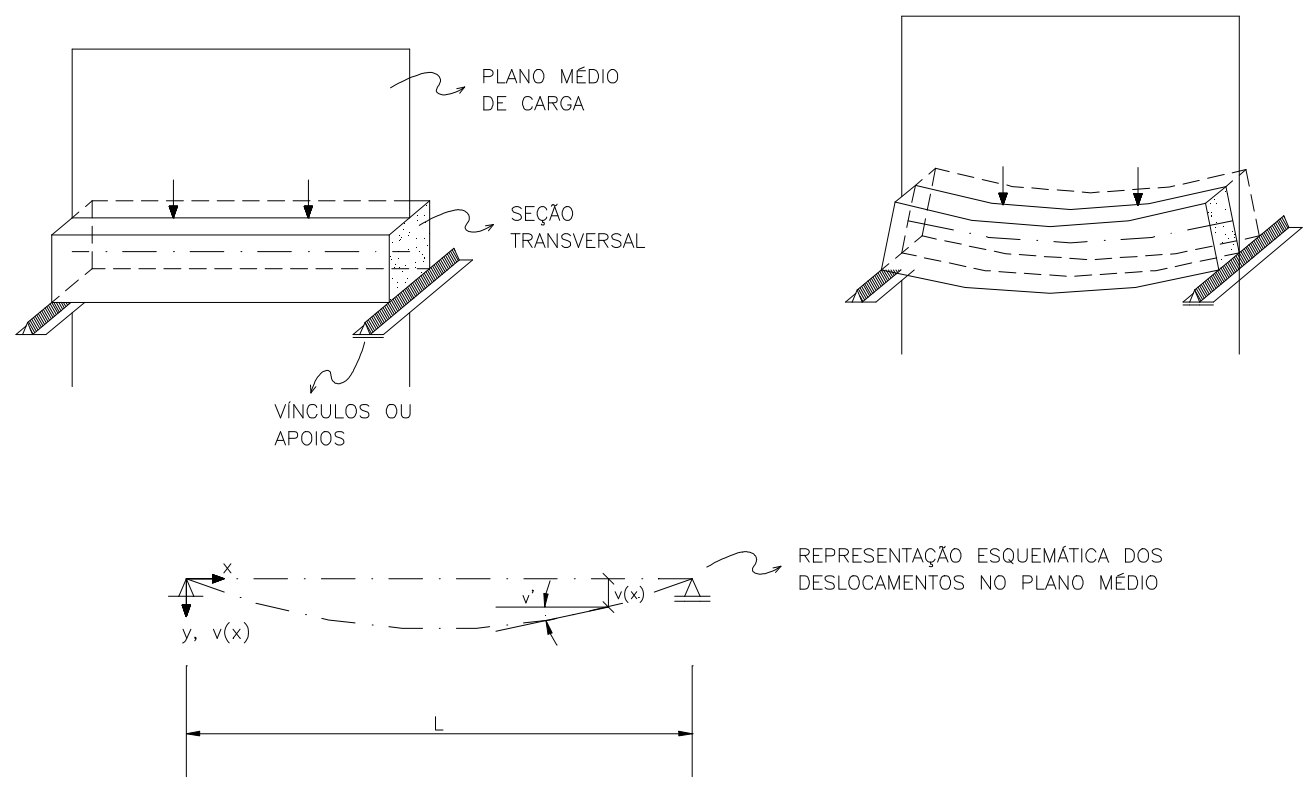

FIGURA 4.1 - Representação esquemática dos deslocamentos no plano médio

Entretanto, a ordem de grandeza dos valores dos deslocamentos em cada ponto ou, de modo alternativo, a resposta da estrutura a uma certa solicitação, depende de fatores como o tipo de material de que é composta e da geometria de sua seção transversal.

Por simplicidade, admite-se que o material seja homogêneo, apresentando as mesmas propriedades em todos os pontos da estrutura, e isótropo, isto é, num ponto as propriedades são as mesmas em qualquer direção. Considere-se ainda que a forma da seção transversal seja invariável $\operatorname{com} x$.

Isto posto, surge o problema de como determinar os valores de $v$ e $v^{\prime}$ de modo a contemplar diferentes situações de carregamento e vinculação. Observa-se que os pares de valores incógnitos constituem, de início, elemento de um espaço solução de dimensão infinita.

Um modo prático de reduzir a dimensão do problema é discretizar o intervalo $[0, L]$ num número finito de pontos de interesse. Entre esses pontos, pode-se admitir que a função real seja aproximada, por exemplo, por uma função polinomial de 
grau $n$ segundo uma interpolação que obedece a idênticas condições de contorno de $v(x)$.

A energia externa é dada, basicamente, pelo produto da carga pelo deslocamento do seu ponto de aplicação. A energia interna é obtida pelo procedimento que se segue.

A energia de deformação acumulada num elemento infinitesimal em estado plano de tensão é dada pela seguinte expressão:

$$
d U=\frac{1}{2}\left(\sigma_{x} \varepsilon_{x}+\sigma_{y} \varepsilon_{y}+\tau_{x y} \gamma_{x y}\right) d x d y d z
$$

pois $\sigma_{z}=\tau_{x z}=\tau_{y z}=0$

Denotando-se o elemento de volume por:

$$
d V=d x d y d z
$$

e indicando-se a energia específica de deformação com a letra $u$ minúscula, segue que:

$$
u=\frac{d U}{d V}=\frac{1}{2}\left(\sigma_{x} \varepsilon_{x}+\sigma_{y} \varepsilon_{y}+\tau_{x y} \gamma_{x y}\right)
$$

Pela Lei de Hooke, tem-se:

$$
\begin{aligned}
& \varepsilon_{x}=\frac{1}{E}\left(\sigma_{x}+v \sigma_{y}\right) \\
& \varepsilon_{y}=\frac{1}{E}\left(\sigma_{y}+v \sigma_{x}\right) \\
& \gamma_{x y}=\frac{1}{G} \tau_{x y}
\end{aligned}
$$

De modo que a expressão da energia específica em função das tensões resulta:

$$
u=\frac{1}{2 E}\left(\sigma_{x}^{2}+\sigma_{y}^{2}-2 v \sigma_{x} \sigma_{y}\right)+\frac{1}{2 G} \tau_{x y}^{2}
$$




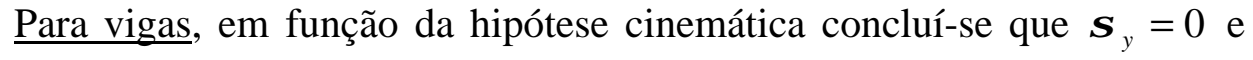
$\gamma_{x y}=0$, que corresponde a desprezar a contribuição da força cortante, então a fórmula da energia específica fica escrita da seguinte maneira:

$$
u=\frac{1}{2 E} \sigma_{x}^{2},
$$

onde $\sigma_{x}=\frac{M y}{I}$, e $M$ é o momento de flexão solicitante na seção genérica da viga.

As expressões anteriores fornecem a energia específica de deformação em cada ponto da viga, isto é, $u$ resulta como função das coordenadas $x, y, z$. Obtém-se então a energia total de deformação por:

$$
U=\int_{V} u d V=\iiint u d x d y d z
$$

$\mathrm{ou}$

$$
U=\frac{1}{2 E} \int_{0}^{L}\left[\int_{A}\left(\frac{M}{I} y\right)^{2} d A\right] d x=\frac{1}{2 E} \int_{0}^{L}\left[\frac{M^{2}}{I^{2}} \int_{A} y^{2} d A\right] d x
$$

ou ainda

$$
U=\frac{1}{2} \int_{0}^{L} \frac{M^{2}}{E I} d x
$$

Observação: Não foi considerada a participação da força normal no desenvolvimento da energia de deformação uma vez que esta têm uma influência muito pequena nas situações de deslocamentos pequenos.

Uma expressão mais interessante para $U$ envolve a curvatura e não o momento.

Nesse sentido, considere-se a barra, de eixo inicialmente reto (ou de grande raio de curvatura), submetida à flexão simples, normal (a flexão oblíqua pode, sempre, ser decomposta em duas flexões normais); originado de um momento $M$ constante ao longo de seu comprimento. Ver figura (4.2): 

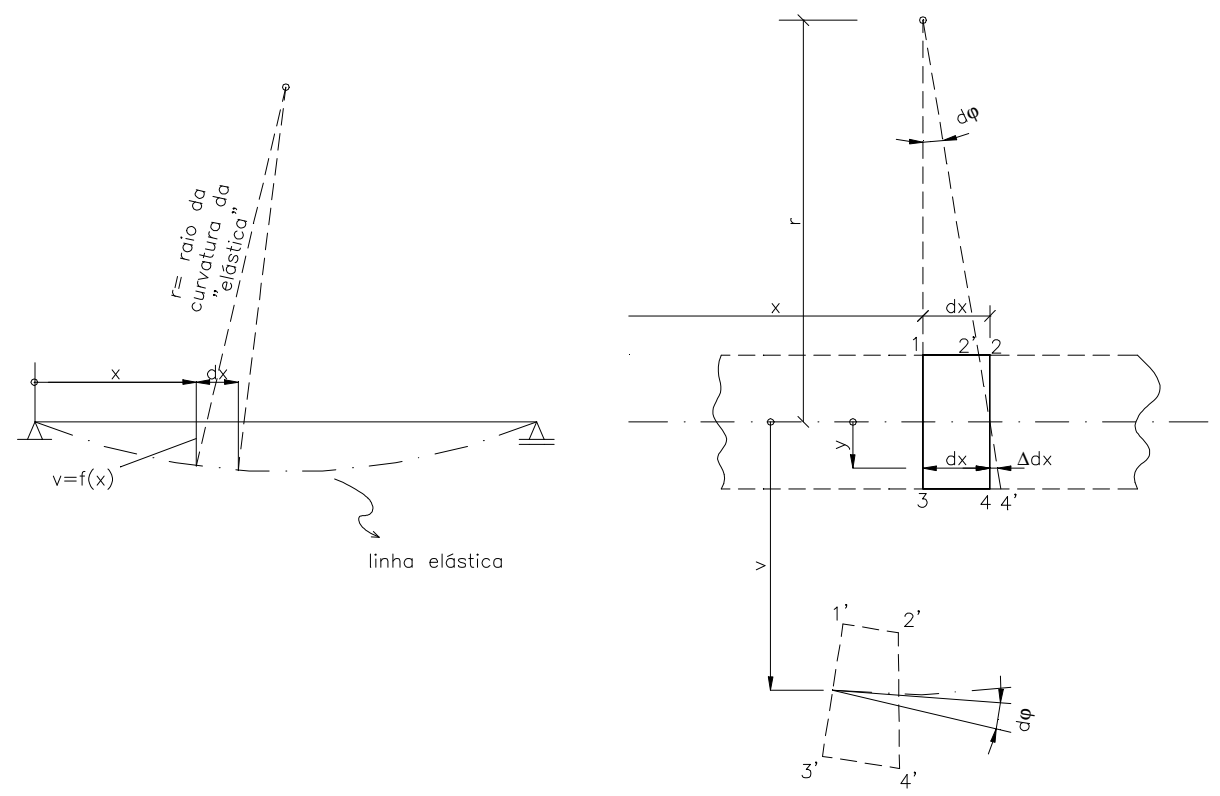

FIGURA 4.2 - Deformação Angular

De acordo com o que se indica na figura, $d x$ é o comprimento das fibras do eixo neutro, e que não varia durante a deformação da barra. Nas fibras que distam $y$ da linha neutra a tensão normal é:

$$
\sigma=\frac{M}{I} y,
$$

e a deformação correspondente, é:

$$
\varepsilon=\frac{\sigma}{E}=\frac{M}{E I} y
$$

As fibras, que distam $y$ da linha neutra, passam a ter, após a deformação, o comprimento:

$$
A B=d x(1+\varepsilon)
$$

Chamando de $\boldsymbol{r}$ o raio de curvatura, correspondente à fibra neutra (onde a tensão normal é nula), após a deformação da barra, vem:

$$
d x=r d \varphi
$$

e: 


$$
d x(1+\varepsilon)=(r+y) d \varphi=r d \varphi(1+\varepsilon),
$$

donde:

$$
\frac{1}{r}=\frac{d \varphi}{d x}=\frac{\varepsilon}{y}=\frac{M}{E I}
$$

A expressão:

$$
\frac{d \varphi}{d x}=\frac{M}{E I}
$$

é aplicável às barras retas e, com suficiente aproximação, às barras curvas de pequena curvatura.

Em coordenadas cartesianas, a expressão da curvatura, em função de $v(x)$, é dada por:

$$
\frac{1}{r}=\frac{d^{2} v}{d x^{2}}\left[1+\left(\frac{d v}{d x}\right)^{2}\right]^{-\frac{3}{2}},
$$

podendo-se desprezar (para os materiais que se deformam pouco), em confronto com a unidade, o quadrado de $d v / d x$ em presença da unidade. Nessas condições, resulta:

$$
\frac{d^{2} v}{d x^{2}} \cong \frac{1}{r}=\frac{d \varphi}{d x}=\frac{M}{E I}
$$

ou mais precisamente:

$$
\frac{d^{2} v}{d x^{2}}= \pm \frac{M}{E I}
$$

onde o sinal positivo corresponde ao caso em que o eixo dos y é orientado para cima, e o negativo o caso em que o eixo dos y é orientado para baixo.

De fato, para o eixo dos $y$ orientado para baixo, o valor de $\varphi$ é positivo no sentido dextrógiro. Nessas condições, quando $x$ cresce, $\varphi$ diminui: se isso ocorre, $d \varphi / d x$ é negativo, devendo-se, então, adotar a equação: 


$$
\frac{d \varphi}{d x}=\frac{d^{2} v}{d x^{2}}=v^{\prime \prime}=-\frac{M}{E I},
$$

daí, vem:

$$
M=-E I \cdot v^{\prime \prime}
$$

Finalmente, a energia interna expressa em função da curvatura da linha elástica é dada por:

$$
U=\frac{1}{2} \int_{0}^{L} E I\left(v^{\prime \prime}\right)^{2} d x
$$

onde $E I$ é a denominada rigidez à flexão e está relacionada tanto ao material quanto à geometria da seção transversal.

Levando-se em conta que a (4.49) exige continuidade para $v$ ”, uma solução aproximada consiste em admitir que a função deslocamento seja dada, no intervalo $0 \leq x \leq L$ por um polinômio do $3^{\circ}$ grau em $x$ :

$$
v(x)=A x^{3}+B x^{2}+C x+D,
$$

sendo as constantes $A, B, C$ e $D$ incógnitas a se determinar.

O procedimento usualmente empregado na interpolação é o de exprimir os coeficientes do polinômio aproximador em função de valores de deslocamento e giro em pontos discretos do intervalo $[0, L]$. No caso, para o polinômio de $3^{\underline{O}}$ grau, definemse dois pares de valores incógnitos pertencentes às seções extremas, $\left(v_{1}, v_{l^{\prime}}\right)$ e $\left(v_{2}, v_{2}{ }^{\prime}\right)$, como indica a figura (4.3). 


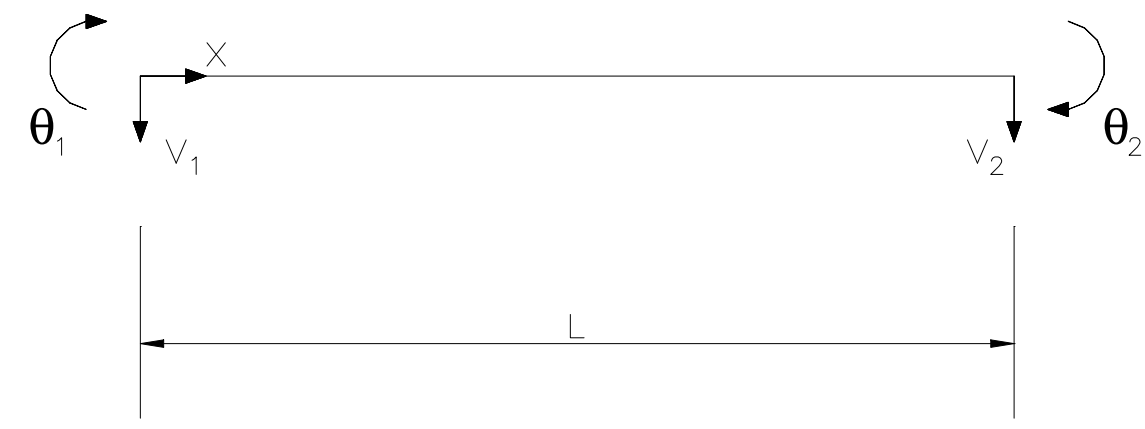

FIGURA 4.3 - Graus de liberdade ou coordenadas adotadas

Fazendo-se:

$$
v_{1}{ }^{\prime}=\theta_{1} \quad \text { e } \quad v_{2}{ }^{\prime}=\theta_{2},
$$

pode-se construir o vetor $v$ de "deslocamentos nodais generalizados", escrito na forma seguinte:

$$
v=\left\{\begin{array}{l}
v_{1} \\
\theta_{1} \\
v_{2} \\
\theta_{2}
\end{array}\right\}
$$

Impondo-se, então, as seguintes condições:

$$
\operatorname{Em} x=0 \quad\left\{\begin{array}{c}
v(0)=v_{1} \\
v^{\prime}(0)=\theta_{1}
\end{array} \quad \text { em } x=L \quad\left\{\begin{array}{c}
v(L)=v_{2} \\
v^{\prime}(L)=\theta_{2}
\end{array},\right.\right.
$$

o polinômio aproximador passa a ser dado por:

$$
v(x)=\left[\begin{array}{c}
-\frac{2}{L^{3}} v_{2}+\frac{1}{L^{2}} \theta_{2} \\
\frac{2}{L^{3}} v_{1}+\frac{1}{L^{2}} \theta_{1}
\end{array}\right] x^{3}+\left[\begin{array}{c}
\frac{3}{L^{2}} v_{2}-\frac{1}{L} \theta_{2} \\
-\frac{2}{L} \theta_{1}-\frac{3}{L^{2}} v_{1}
\end{array}\right] x^{2}+\theta_{1} x+v_{1}
$$

Por outro lado, o "funcional" $\pi$ é, neste caso, dado por: 


$$
\pi=\frac{1}{2} \int_{0}^{L} E I\left(v^{\prime \prime}\right)^{2} d x+\Psi ; \text { e } \quad \Psi=-\mathrm{T},
$$

onde: $\Psi$ é a energia externa e $\mathbf{T}$ é o trabalho das forças externas.

O Trabalho das forças externas é dado pelo produto da carga pelo deslocamento do seu ponto de aplicação, e pode ser representado como:

$$
T=\sum_{i=1}^{4} P_{i} \cdot v_{i}=P . v
$$

onde $\quad P=\left[\begin{array}{c}P_{1} \\ P_{2} \\ P_{3} \\ P_{4}\end{array}\right]$

$P$ representa o vetor de cargas nodais equivalentes da estrutura $\left(P \in \mathrm{R}^{4}\right)$. Tendo-se em vista que neste caso:

$$
v^{\prime \prime}(x)=6 A x+2 B,
$$

e, que a rigidez EI é constante, pois o material da viga é homogêneo e a seção transversal não varia ao longo do comprimento da viga, resulta a seguinte expressão para $\pi$ :

$$
\begin{aligned}
\pi\left(v_{1}, \theta_{1}, v_{2}, \theta_{2}\right)= & E I\left[\frac{6}{L^{3}} v_{2}^{2}+\frac{6}{L^{3}} v_{1}^{2}+\frac{2}{L} \theta_{2}^{2}+\frac{2}{L} \theta_{1}^{2}-\frac{6}{L^{2}} v_{2} \theta_{2}+\right. \\
& \left.+\frac{6}{L^{2}} v_{1} \theta_{1}+\frac{2}{L} \theta_{1} \theta_{2}-\frac{12}{L^{3}} v_{1} v_{2}+\frac{6}{L^{2}} v_{1} \theta_{2}-\frac{6}{L^{2}} v_{2} \theta_{1}\right]+\Psi
\end{aligned}
$$

Nota-se que $\pi$ é função de quatro variáveis, e que a determinação destas resulta da imposição do teorema fundamental, que associa a condição deformada em equilíbrio com o mínimo da energia total. Assim, o passo seguinte é a minimização de $\pi\left(v_{1}, \theta_{1}, v_{2}, \theta_{2}\right)$.

Como $\pi$ é uma função quadrática $\left(\pi: \mathrm{R}^{4} \rightarrow \mathrm{R}\right)$, por conveniência pode-se escrevê-la na seguinte forma matricial: 


$$
\pi(V)=\frac{1}{2} v^{T} R v-P^{T} v
$$

onde: $R \in \mathrm{R}^{4 \times 4}$ é a matriz hessiana que recebe o nome de matriz de rigidez da estrutura.

O vetor gradiente de $\pi$ é dado por:

$$
\nabla \pi(v)=\left[\begin{array}{l}
\frac{\partial \pi}{\partial v_{1}} \\
\frac{\partial \pi}{\partial \theta_{1}} \\
\frac{\partial \pi}{\partial v_{2}} \\
\frac{\partial \pi}{\partial \theta_{2}}
\end{array}\right]=E I\left[\begin{array}{l}
\frac{12}{L^{3}} v_{1}+\frac{6}{L^{2}} \theta_{1}-\frac{12}{L^{3}} v_{2}+\frac{6}{L^{2}} \theta_{2} \\
\frac{4}{L} \theta_{1}+\frac{6}{L^{2}} v_{1}+\frac{2}{L} \theta_{2}-\frac{6}{L^{2}} v_{2} \\
\frac{12}{L^{3}} v_{2}-\frac{6}{L^{2}} \theta_{2}-\frac{12}{L^{3}} v_{1}-\frac{6}{L^{2}} \theta_{1} \\
\frac{4}{L} \theta_{2}-\frac{6}{L^{2}} v_{2}+\frac{2}{L} \theta_{1}+\frac{6}{L^{2}} v_{1}
\end{array}\right]+\left[\begin{array}{l}
\frac{\partial \Psi}{\partial v_{1}} \\
\frac{\partial \Psi}{\partial \theta_{1}} \\
\frac{\partial \Psi}{\partial v_{2}} \\
\frac{\partial \Psi}{\partial \theta_{2}}
\end{array}\right]
$$

Note que: $\frac{\partial \pi}{\partial v_{i}}: \mathrm{R}^{4} \rightarrow \mathrm{R} \quad$, em particular é uma função linear, pois $\pi$ é quadrática, e que:

$$
\frac{\partial \Psi}{\partial v_{i}}=-\frac{\partial T}{\partial v_{i}}=-P
$$

$\mathrm{Na}$ forma matricial o vetor gradiente resulta:

$$
\nabla \pi(v)=R v-P, \text { sendo ainda } \nabla \pi(0)=-P
$$

Por sua vez o segundo gradiente de $\pi$ é dado por:

$$
\nabla^{2} \pi(v)=R=\left[\begin{array}{cccc}
\frac{\partial^{2} \pi}{\partial^{2} v_{1}} & \frac{\partial^{2} \pi}{\partial \theta_{1} \partial v_{1}} & \frac{\partial^{2} \pi}{\partial v_{2} \partial v_{1}} & \frac{\partial^{2} \pi}{\partial \theta_{2} \partial v_{1}} \\
\frac{\partial^{2} \pi}{\partial v_{1} \partial \theta_{1}} & \frac{\partial^{2} \pi}{\partial^{2} \theta_{1}} & \frac{\partial^{2} \pi}{\partial v_{2} \partial \theta_{1}} & \frac{\partial^{2} \pi}{\partial \theta_{2} \partial \theta_{1}} \\
\frac{\partial^{2} \pi}{\partial v_{1} \partial v_{2}} & \frac{\partial^{2} \pi}{\partial \theta_{1} \partial v_{2}} & \frac{\partial^{2} \pi}{\partial^{2} v_{2}} & \frac{\partial^{2} \pi}{\partial \theta_{2} \partial v_{2}} \\
\frac{\partial^{2} \pi}{\partial v_{1} \partial \theta_{2}} & \frac{\partial^{2} \pi}{\partial \theta_{1} \partial \theta_{2}} & \frac{\partial^{2} \pi}{\partial v_{2} \partial \theta_{2}} & \frac{\partial^{2} \pi}{\partial^{2} \theta_{2}}
\end{array}\right]=
$$




$$
=E I\left[\begin{array}{cccc}
\frac{12}{L^{3}} & \frac{6}{L^{2}} & -\frac{12}{L^{3}} & \frac{6}{L^{2}} \\
\frac{6}{L^{2}} & \frac{4}{L} & -\frac{6}{L^{2}} & \frac{2}{L} \\
-\frac{12}{L^{3}} & -\frac{6}{L^{2}} & \frac{12}{L^{3}} & -\frac{6}{L^{2}} \\
\frac{6}{L^{2}} & \frac{2}{L} & -\frac{6}{L^{2}} & \frac{4}{L}
\end{array}\right]
$$

\section{Observações:}

1a) É sempre possível, quaisquer que sejam as coordenadas, gerar a matriz de rigidez correspondente; entretanto, essa matriz só pode ser gerada diretamente se, para deslocamentos prescritos segundo as coordenadas, a estrutura resultar determinada, ou conhecida, a priori; ou seja, se existirem vínculos, segundo as coordenadas ou não, em número suficiente para se determinar a posição da estrutura, ou elemento.

2a) A matriz de rigidez permite conhecer as forças segundo as coordenadas a partir do conhecimento dos deslocamentos segundo essas coordenadas.

3a) A matriz de rigidez $R$ é simétrica em relação a diagonal principal, isto é, $\quad R_{i j}=R_{j i}$, o que também é consistente com o teorema de Betti. Os termos da diagonal principal são não negativos, ou seja, $R_{i i} \geq 0$.

É interessante analisar a construção da matriz de rigidez para o caso de se ter mais de um elemento.

Considerando-se que a energia total $\pi$ da barra é composta pelas contribuições da energia dos elementos $I$ e $I I$ nos quais ela é discretizada, vale a relação:

$$
\pi=\pi_{I}+\pi_{I I}
$$

A energia e a matriz de rigidez do elemento $I$ são dadas respectivamente por:

$$
\pi_{I}=\frac{1}{2} \delta_{I}^{T} R_{I} \delta_{I} \quad R_{I}=\left[\begin{array}{cc}
R_{1}^{I} & R_{12}^{I} \\
R_{12}^{I} & R_{2}^{I}
\end{array}\right]
$$


Analogamente, a energia e a matriz de rigidez do elemento II é dada por:

$$
\pi_{I I}=\frac{1}{2} \delta_{I I}^{T} R_{I I} \delta_{I I} \quad R_{I I}=\left[\begin{array}{cc}
R_{1}^{I I} & R_{12}^{I I} \\
R_{12}^{I I} & R_{2}^{I I}
\end{array}\right],
$$

onde os vetores $\delta_{I}=\left(\begin{array}{l}x_{1} \\ x_{2}\end{array}\right)$ e $\delta_{I I}=\left(\begin{array}{l}x_{2} \\ x_{3}\end{array}\right)$ reúnem os deslocamentos nodais $x_{i}$ de cada elemento, sendo que $x_{i}$ representa os dois deslocamentos generalizados da extremidade do elemento.

A partir das expressões anteriores, pode-se calcular a energia total $\pi$ da barra, pela soma da energia de cada elemento:

$$
\begin{aligned}
2 \pi_{I}=\left(\begin{array}{ll}
x_{1}^{T} & x_{2}^{T}
\end{array}\right)\left[\begin{array}{ll}
R_{1}^{I} & R_{12}^{I} \\
R_{12}^{I} & R_{2}^{I}
\end{array}\right]\left(\begin{array}{l}
x_{1} \\
x_{2}
\end{array}\right)= \\
=x_{1}^{T} R_{1}^{I} x_{1}+2 x_{1}^{T} R_{12}^{I} x_{2}+x_{2}^{T} R_{2}^{I} x_{2} \\
2 \pi_{I I}=\left(\begin{array}{ll}
x_{2}^{T} & x_{3}^{T}
\end{array}\right)\left[\begin{array}{ll}
R_{1}^{I I} & R_{12}^{I I} \\
R_{12}^{I I} & R_{2}^{I I}
\end{array}\right]\left(\begin{array}{l}
x_{2} \\
x_{3}
\end{array}\right)= \\
=x_{2}^{T} R_{1}^{I I} x_{2}+2 x_{2}^{T} R_{12}^{I I} x_{3}+x_{3}^{T} R_{2}^{I I} x_{3}
\end{aligned}
$$

Somando-se as duas parcelas, tem-se:

$$
\begin{aligned}
2 \pi= & x_{1}^{T} R_{1}^{I} x_{1}+2 x_{1}^{T} R_{12}^{I} x_{2}+x_{2}^{T}\left(R_{2}^{I}+R_{1}^{I I}\right) x_{2}+ \\
& +2 x_{2}^{T} R_{12}^{I I} x_{3}+x_{3}^{T} R_{2}^{I I} x_{3}
\end{aligned}
$$

Utilizando-se a seguinte propriedade da Álgebra Linear:

$$
\left(\begin{array}{ll}
x^{T} & y^{T}
\end{array}\right)\left[\begin{array}{cc}
A & C \\
C^{T} & B
\end{array}\right]\left(\begin{array}{l}
x \\
y
\end{array}\right)=x^{T} A x+2 x^{T} C y+y^{T} B y
$$

estendendo-a para o caso em questão e colocando-se a energia total na forma matricial, $\pi=\frac{1}{2} \delta^{T} R \delta$, obtém-se: 


$$
\delta=\left(\begin{array}{l}
x_{1} \\
x_{2} \\
x_{3}
\end{array}\right) \quad R=\left[\begin{array}{ccc}
R_{1}^{I} & R_{12}^{I} & 0 \\
R_{12}^{I} & \left(R_{2}^{I}+R_{1}^{I I}\right) & R_{12}^{I I} \\
0 & R_{12}^{I I} & R_{2}^{I I}
\end{array}\right]
$$

O que se observa é uma superposição da matriz de rigidez do segundo elemento sobre a do primeiro nas posições correspondentes às coordenadas envolvidas no nó comum a ambos os elementos. Dessa forma, é possível montar a matriz de rigidez para vigas simplesmente calculando uma matriz de rigidez para cada elemento que se criou e, depois, fazendo-se a superposição das mesmas uma vez conhecida a ordem dos elementos.

Uma vez construída a matriz de rigidez da estrutura e o vetor de cargas devido ao carregamento externo na estrutura, o passo seguinte é a determinação de todos os deslocamentos nas extremidades dos elementos a partir da minimização do funcional $\pi$.

Após a obtenção dos deslocamentos nas extremidades dos elementos através da minimização do funcional $\pi$, pode-se calcular os esforços nas coordenadas correspondentes, para cada elemento, através do vetor de cargas, da matriz de rigidez e do vetor de deslocamentos dos mesmos.

Os esforços para cada elemento são calculados da seguinte forma:

$$
\left\{\begin{array}{l}
P_{e 1} \\
P_{e 2} \\
P_{e 3} \\
P_{e 4}
\end{array}\right\}=\left\{\begin{array}{l}
P_{1} \\
P_{2} \\
P_{3} \\
P_{4}
\end{array}\right\}+E I\left[\begin{array}{cccc}
\frac{12}{L^{3}} & \frac{6}{L^{2}} & -\frac{12}{L^{3}} & \frac{6}{L^{2}} \\
\frac{6}{L^{2}} & \frac{4}{L} & -\frac{6}{L^{2}} & \frac{2}{L} \\
-\frac{12}{L^{3}} & -\frac{6}{L^{2}} & \frac{12}{L^{3}} & -\frac{6}{L^{2}} \\
\frac{6}{L^{2}} & \frac{2}{L} & -\frac{6}{L^{2}} & \frac{4}{L}
\end{array}\right]\left\{\begin{array}{c}
v_{1} \\
\theta_{1} \\
v_{2} \\
\theta_{2}
\end{array}\right\}
$$

\section{2 - Treliça Espacial}

A treliça espacial é composta pela união de elementos ou barras, articulados em nós; esses nós possuem 3 deslocamentos independentes possíveis e a eles podem-se aplicar 3 forças independentes, sendo portanto interessante adotar 3 
coordenadas por nó. Por mera questão de sistematização, sem que isso implique em perda de generalidade, a numeração dessas coordenadas mantem a mesma sequência de numeração dos nós, ou seja, ao nó $t$ genérico, corresponderão as coordenadas: $3 t-2 ; 3 t$ $1 ; 3 t$

Para sistematizar também a orientação, aproveitando a definição do sistema global de referência $O x y z$, a coordenada $t_{1}$ terá a orientação do eixo $x$, a $t_{2}$ a do $y$ e a $t_{3}$ a do $z$, conforme figura (4.4).
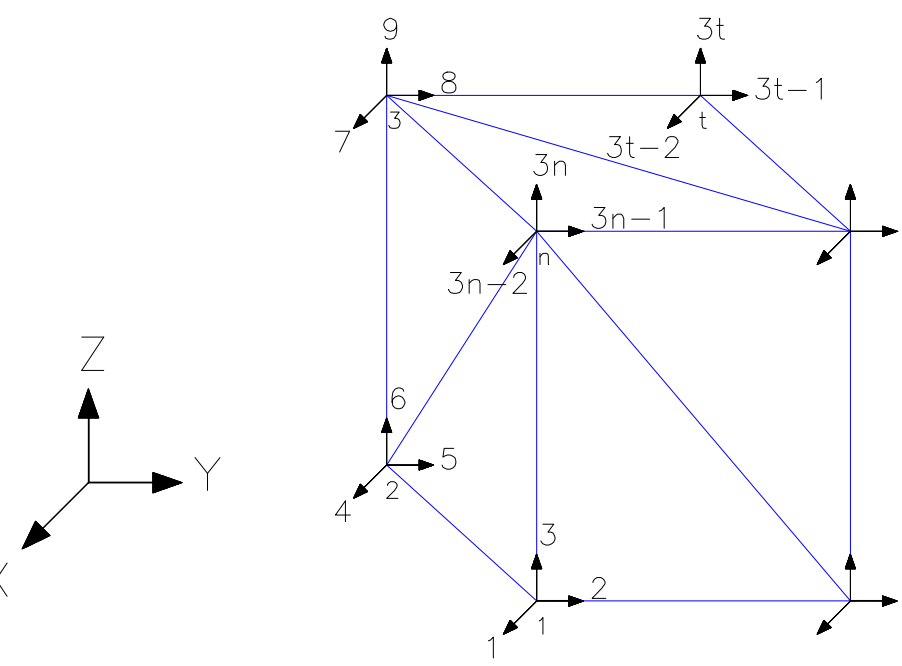

FIGURA 4.4 - Coordenadas Globais

Em relação às coordenadas globais serão definidos os vetores:

$F \rightarrow$ vetor das forças nodais

$u \rightarrow$ vetor dos deslocamentos nodais

Para uma barra genérica i, assumida como elemento, padronizado por opção, com um nó inicial $j$ e um nó final $k$, tem-se 3 esforços, ou 3 deslocamentos independentes por extremidade, sendo interessante criar, então, 3 coordenadas por extremidade, conforme indica a figura (4.5): 


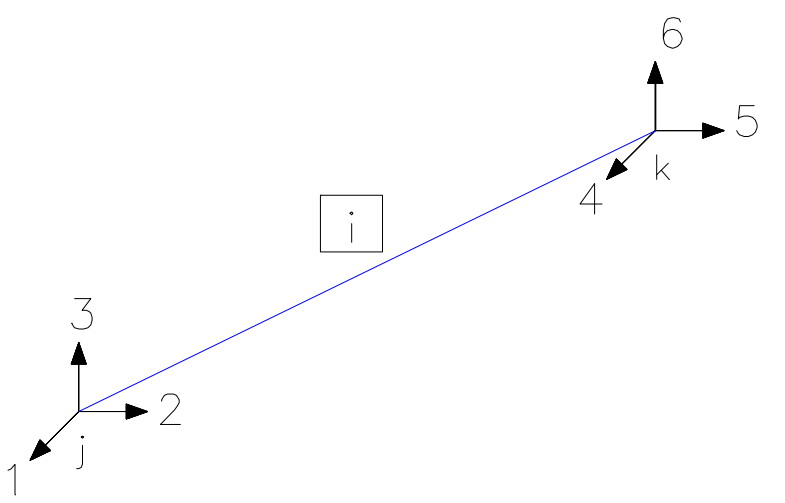

FIGURA 4.5 - Coordenadas Locais segundo o Sistema Global

Por outro lado, para introduzir dados e manusear resultados, é mais interessante trabalhar com as coordenadas da figura (4.6), com uma coordenada associada à extremidade inicial e uma à extremidade final. Cada uma dessas coordenadas tem também sua orientação associada sequencialmente às dos eixos $x, y$ e $z$ do sistema local de referência associado a barra $i$.

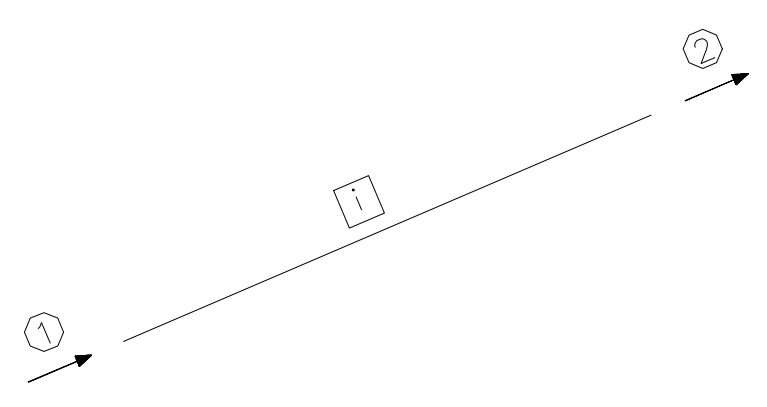

FIGURA 4.6 - Coordenadas Locais da Barra

Para tirar proveito de ambos os conjuntos de coordenadas é usual trabalhar com os dois sistemas. Para evitar confusão de notação atribui-se aos vetores e matrizes associados ao elemento, um índice adicional; assim, para as coordenadas da figura (4.6), mais naturalmente associadas ao elemento, atribui-se o índice $\boldsymbol{e}$ obtendo-se:

$\delta_{e_{i}} \rightarrow$ deslocamentos nas coordenadas locais associadas ao elemento

$P_{e_{i}} \rightarrow$ forças nas coordenadas locais associadas ao elemento 
$r_{e_{i}} \rightarrow$ matriz de rigidez do elemento nas coordenadas locais associadas ao elemento.

Para as coordenadas locais da figura (4.5), mais diretamente relacionadas às direções do sistema global de referência, atribui-se o índice $g$. Assim, tem-se:

$\delta_{g_{i}} \rightarrow$ deslocamentos nas coordenadas locais associadas ao sistema global de referência

$P_{g_{i}} \rightarrow$ forças nas coordenadas locais associadas ao sistema global de referência

$\mathrm{r}_{g_{i}} \rightarrow$ matriz de rigidez do elemento nas coordenadas locais associadas ao sistema global de referência.

É extremamente simples relacionar as matrizes e vetores desses dois sistemas locais utilizando a formulação matricial que envolve a matriz de incidência.

Relacionando o vetor $\delta_{g_{i}}$ dos deslocamentos da "estrutura" ao vetor $\delta_{e_{i}}$ dos deslocamentos do "elemento", pode-se definir uma matriz de incidência cinemática $\beta_{e_{i}}$

$$
\delta_{e i}=\beta_{e i} \delta_{e i}
$$

Essa matriz $\beta$ tem sempre 12 elementos e é obtida facilmente por:

$$
\beta_{e i}=\left[\begin{array}{cccccc}
C x & C y & C z & 0 & 0 & 0 \\
0 & 0 & 0 & C x & C y & C z
\end{array}\right],
$$

onde $C_{x}, C_{y}$ e $C_{z}$ são os cossenos diretores na direção $x, y$, e $z$ respectivamente.

A matriz de rigidez da estrutura $R$ pode ser gerada através do seguinte procedimento:

A matriz $r_{e_{i}}$ é determinada diretamente através da definição: 


$$
r_{e i}=\frac{E A}{L}\left[\begin{array}{cc}
1 & -1 \\
-1 & 1
\end{array}\right]
$$

onde: $E=$ módulo de elasticidade longitudinal

$A=$ área da barra

$L=$ comprimento da barra

Tendo $\beta_{e_{i}}$ e $r_{e_{i}}$, para a "estrutura" com um só "elemento", obtém-se:

$$
r_{g_{i}}=\beta_{e i}^{T} r_{e i} \beta_{e i}
$$

Efetuando-se essas operações matriciais, obtém-se:

$$
r_{g_{i}}=\frac{E A}{L}\left[\begin{array}{cccccc}
C x^{2} & C x C y & C x C z & -C x^{2} & -C x C y & -C x C z \\
& C y^{2} & C y C z & -C y C x & -C y^{2} & -C y C z \\
& & C z^{2} & -C z C x & -C z C y & -C z^{2} \\
& & & C x^{2} & C x C y & C x C z \\
& \text { simetrico } & & & C y^{2} & C y C z \\
& & & & & C z^{2}
\end{array}\right]
$$

Genericamente, a contribuição $R_{i}$ da barra $i$ para a matriz de rigidez $R$ da estrutura pode ser obtida a partir da seguinte expressão:

$$
R_{i}=\beta_{g_{i}}^{T} r_{g_{i}} \beta_{g_{i}}
$$

Uma vez gerada a matriz de rigidez $R$ da estrutura e conhecido o vetor das forças nodais $F$, pode-se determinar o vetor de deslocamentos da estrutura resolvendo-se a minimização:

$$
\min \pi=\frac{1}{2} u^{n^{T}} R u^{n}-u^{n^{T}} F
$$

Com isso determina-se $\delta_{g_{i}}$ tal que: 


$$
\delta_{g_{i}}=\beta_{g_{i}} u
$$

Obtendo-se assim o deslocamento na extremidade da barra $\delta_{e_{i}}$, segundo o sistema de referência local:

$$
\delta_{e i}=\beta_{e i} \delta_{g_{i}}
$$

Calculado esses deslocamentos, pode-se determinar os esforços nas extremidades das barras pela fórmula direta:

$$
P_{e i}=r_{e i} \delta_{e i}
$$

\section{3 - Pórticos}

A modelação para um pórtico é muito semelhante àquela desenvolvida para as vigas. Portanto a resolução de pórticos pode ser feita por:

$$
\min \pi \quad \text { ou } \min \left\{\frac{1}{2} x^{T} R x-P^{T} x\right\},
$$

onde: $R$ é a matriz de rigidez global e $P$ é o vetor de cargas nodais da estrutura.

A técnica de geração de $R$ e $P$ consiste em considerar a contribuição das matrizes de rigidez de cada elemento e, calcular as forças nodais equivalentes em função do carregamento externo, assim como realizado para as vigas.

Uma vez montada a matriz de rigidez global do pórtico $(R)$ e calculada as forças nodais equivalentes $(P)$, os deslocamentos serão determinados através da minimização do funcional da energia total do pórtico, através do emprego de métodos de otimização.

A figura (4.7) indica os conjuntos de graus de liberdade ou coordenadas globais e locais para um elemento de pórtico: 

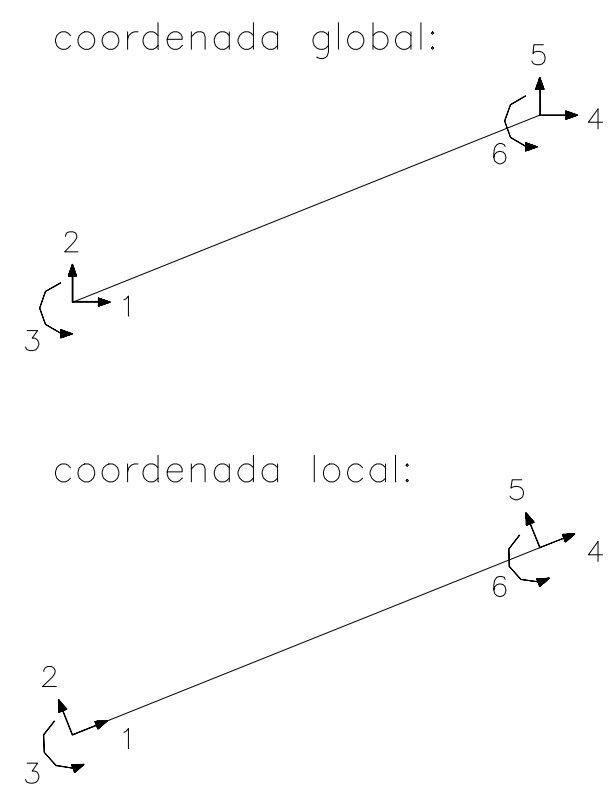

FIGURA 4.7 - Coordenadas dos Elementos

A matriz de rigidez do elemento segundo o sistema local é dado por:

$$
R=\left[\begin{array}{cccccc}
\frac{E A}{L} & 0 & 0 & -\frac{E A}{L} & 0 & 0 \\
0 & \frac{12 E I}{L^{3}} & \frac{6 E I}{L^{2}} & 0 & -\frac{12 E I}{L^{3}} & \frac{6 E I}{L^{2}} \\
0 & \frac{6 E I}{L^{2}} & \frac{4 E I}{L} & 0 & -\frac{6 E I}{L^{2}} & \frac{2 E I}{L} \\
-\frac{E A}{L} & 0 & 0 & \frac{E A}{L} & 0 & 0 \\
0 & -\frac{12 E I}{L^{3}} & -\frac{6 E I}{L^{2}} & 0 & \frac{12 E I}{L^{3}} & -\frac{6 E I}{L^{2}} \\
0 & \frac{6 E I}{L^{2}} & \frac{2 E I}{L} & 0 & -\frac{6 E I}{L^{2}} & \frac{4 E I}{L}
\end{array}\right]
$$

Definindo-se a matriz de rigidez de um elemento, pode-se determinar a matriz de rigidez global da estrutura a partir da contribuição das matrizes de cada elemento observando a correspondência entre a numeração local e global de coordenadas. O mesmo vale para a geração do vetor de cargas nodais equivalentes. Então, através do mesmo procedimento adotado para o caso de vigas, segue-se a otimização da energia do pórtico determinando-se os deslocamentos nodais e, posteriormente, calculando-se os esforços nas coordenadas correspondentes. 


\section{4 - Treliça Plana com não-linearidade geométrica}

Em problemas envolvendo grandes deslocamentos, deve-se levar em conta que o equilíbrio estabelece-se na posição, ou configuração, deslocada da estrutura, a qual não pode mais ser confundida com a configuração inicial.

Na chamada descrição Lagrangiana Total, toda a análise pode ser realizada tomando-se por referência a configuração inicial e, nesse sentido, forças e deslocamentos medidos na situação deslocada terão componentes segundo as direções do eixo e transversal a ele na situação inicial. Por esse motivo é necessário associar à barra na configuração inicial, dois graus de liberdade por nó, no caso plano.

Mesmo que o material se apresente em regime elástico-linear, os problemas envolvendo grandes deslocamentos são intrinsecamente não-lineares, uma vez que a rigidez da estrutura é função do próprio campo de deslocamentos. Assim sendo, a condição de equilíbrio resulta não-linear e uma estratégia de solução consiste em linearizá-la e estabelecer um procedimento incremental-iterativo para sucessivamente reduzir os erros induzidos pela linearização.

Na linearização em questão, gera-se uma matriz de rigidez tangente da estrutura, a qual vai sendo sucessivamente atualizada no processo de resolução.

É importante observar que a linearização exige que o carregamento total seja aplicado em etapas para que o erro gerado em cada etapa não seja tão expressivo. As iterações se desenvolvem dentro de cada etapa ou passo de carregamento para que o erro possa ser reduzido e a matriz de rigidez atualizada de acordo com a evolução dos deslocamentos.

Uma abordagem completa da questão não-linear foge dos limites deste texto mas pode ser encontrada em PAULA (1997), ou em PROENÇA (1998).

No que segue apresentam-se os passos essenciais para a dedução da matriz de rigidez tangente pela aplicação do Princípio dos Trabalhos Virtuais que, neste caso, equivale à imposição da estacionariedade da energia potencial total.

Considere-se o elemento de barra de treliça plana na configuração de referência e um sistema local de coordenadas mostrado na figura (4.8), com quatro graus de liberdade, sendo dois por nó, correspondentes aos deslocamentos na direção do eixo da barra e transversal a ele. O elemento possui, na configuração inicial, área de seção transversal $A_{0}$ e comprimento $L_{0}$. O material é considerado elástico linear e as deformações sofridas são também consideradas pequenas. 


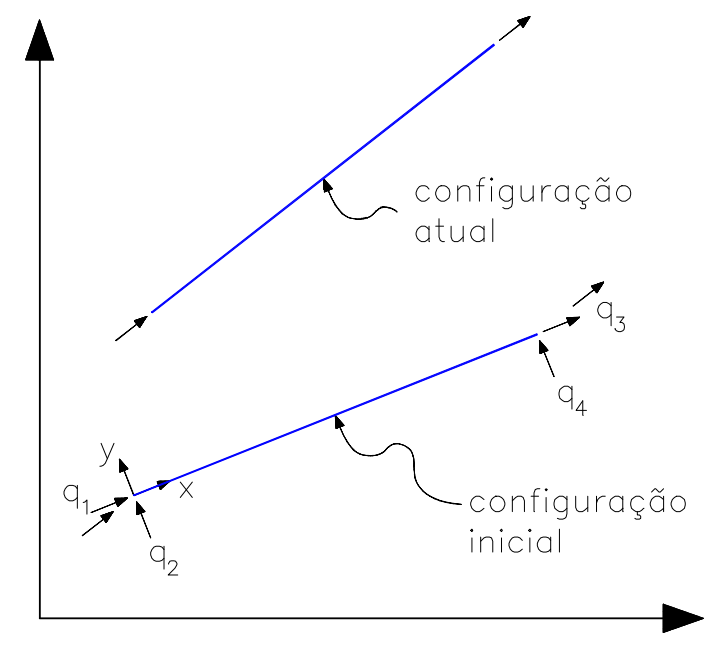

FIGURA 4.8 - Elemento finito de treliça plana

Para o elemento finito mostrado na figura (4.8), os deslocamentos são interpolados de maneira usual por:

$$
u=\left\{\begin{array}{l}
u_{1} \\
u_{2}
\end{array}\right\}=M q,
$$

Sendo:

$\mathrm{u}_{1}=$ componente do deslocamento na direção do eixo da barra

$\mathrm{u}_{2}=$ componente do deslocamento na direção transversal ao eixo da barra

$$
M=\left[\begin{array}{cccc}
N_{1} & 0 & N_{3} & 0 \\
0 & N_{2} & 0 & N_{4}
\end{array}\right],
$$

e as funções interpoladoras e o vetor dos deslocamentos nodais são, respectivamente:

$$
\begin{aligned}
& N_{1}(X)=N_{2}(X)=\left(1-\frac{x}{L_{0}}\right) \\
& N_{3}(X)=N_{4}(X)=\frac{x}{L_{0}}
\end{aligned}
$$




$$
q^{T}=\left\{\begin{array}{llll}
q_{1} & q_{2} & q_{3} & q_{4}
\end{array}\right\}
$$

Observa-se que $X$ é uma coordenada local com origem numa das extremidades da barra.

$$
\text { Como } \frac{\partial u_{i}}{\partial y}=0 \quad(i=1,2) \text { o gradiente dos deslocamentos pode então ser }
$$

expresso por:

$$
\nabla u=\left[\begin{array}{l}
\frac{\partial u_{1}}{\partial X} \\
\frac{\partial u_{2}}{\partial X}
\end{array}\right] \Rightarrow \begin{gathered}
\frac{\partial u_{1}}{\partial X}=-\frac{1}{L_{0}} q_{1}+\frac{1}{L_{0}} q_{3} \\
\frac{\partial u_{2}}{\partial X}=-\frac{1}{L_{0}} q_{2}+\frac{1}{L_{0}} q_{4}
\end{gathered}
$$

ou na forma matricial por:

$$
\nabla u=G q \quad \text { onde } \quad G=\frac{1}{L_{0}}\left[\begin{array}{cccc}
-1 & 0 & 1 & 0 \\
0 & -1 & 0 & 1
\end{array}\right]
$$

Neste tipo de descrição é mais conveniente trabalhar com o tensor de deformação de Green, em lugar do tensor de deformação linear e sua relação com o gradiente do campo de deslocamentos é dado por:

$$
\varepsilon=\frac{1}{2}\left(\nabla u+\nabla u^{T}+\nabla u^{T} \nabla u\right)
$$

No caso, a deformação de Green tem uma única componente ativa, $\varepsilon_{11}$, dada por:

$$
\varepsilon_{11}=\left(\frac{\partial u_{1}}{\partial X}\right)+\frac{1}{2}\left[\left(\frac{\partial u_{1}}{\partial X}\right)^{2}+\left(\frac{\partial u_{2}}{\partial X}\right)^{2}\right]
$$

Os deslocamentos virtuais são interpolados de modo análogo como $\delta u=M \delta q$. A deformação virtual de Green resulta: 


$$
\delta \varepsilon_{11}=\left(\frac{\partial \delta u_{1}}{\partial X}\right)+\left(\frac{\partial \delta u_{1}}{\partial X}\right)\left(\frac{\partial u_{1}}{\partial X}\right)+\left(\frac{\partial \delta u_{2}}{\partial X}\right)\left(\frac{\partial u_{2}}{\partial X}\right)
$$

Efetuando-se as derivadas da equação acima e escrevendo-se na forma matricial tem-se:

$$
\begin{aligned}
& \delta \varepsilon_{11}=\left\{\left(\begin{array}{llll}
-\frac{1}{L_{0}} & 0 & \frac{1}{L_{0}} & 0
\end{array}\right)+\right. \\
& \left.+\frac{1}{L_{0}^{2}}\left[\begin{array}{llll}
-\left(q_{3}-q_{1}\right) & -\left(q_{4}-q_{2}\right) & \left(q_{3}-q_{1}\right) & \left(q_{4}-q_{2}\right)
\end{array}\right]\right\}\left\{\begin{array}{c}
\delta q_{1} \\
\delta q_{2} \\
\delta q_{3} \\
\delta q_{4}
\end{array}\right\}
\end{aligned}
$$

Essa última relação pode ser representada por:

$$
\delta \varepsilon_{11}=B \delta q \quad \text { tal que } \quad B=B_{0}+B_{L}
$$

onde:

$$
\begin{aligned}
& B_{0}=\left[\begin{array}{llll}
-\frac{1}{L_{0}} & 0 & \frac{1}{L_{0}} & 0
\end{array}\right] \\
& B_{L}=\frac{1}{L_{0}^{2}}\left[\begin{array}{llll}
-\left(q_{3}-q_{1}\right) & -\left(q_{4}-q_{2}\right) & \left(q_{3}-q_{1}\right) & \left(q_{4}-q_{2}\right)
\end{array}\right]
\end{aligned}
$$

Normalmente em problemas não-lineares a relação constitutiva é expressa em termo de velocidades, ou taxas, de tensão e de deformação, de modo a possibilitar a consideração de modelos não-elásticos. Dessa forma, a equação constitutiva para problemas envolvendo pequenas deformações e grandes deslocamentos, pode ser escrita por:

$$
\dot{S}=D \dot{\varepsilon},
$$

onde $D$ é a matriz constitutiva do material.

Nota-se que na (4.98) o tensor das tensões é o tensor de Piola-Kirchhoff de segunda espécie, conjugado ao tensor de deformações de Green. 
O gradiente dos deslocamentos virtuais é dado por:

$$
\nabla \delta u=G \delta q
$$

A taxa de deformação de Green para o elemento de barra de treliça plana é, por sua vez, dada por:

$$
\dot{\varepsilon}_{11}=\left(\frac{\partial \dot{u}_{1}}{\partial X}\right)+\left(\frac{\partial u_{1}}{\partial X}\right)\left(\frac{\partial \dot{u}_{1}}{\partial X}\right)+\left(\frac{\partial u_{2}}{\partial X}\right)\left(\frac{\partial \dot{u}_{2}}{\partial X}\right)=B \dot{q},
$$

e a taxa de deformação virtual de Green por:

$$
\delta \dot{\varepsilon}_{11}^{T}=\delta q^{T} G^{T} G \dot{q}
$$

Para a obtenção direta da rigidez tangente o PTV expresso em taxas é mais conveniente e nesta formulação assume a forma:

$$
\frac{d}{d t} \int_{V_{0}} r_{0}^{T} \delta u d V_{0}=\frac{d}{d t}\left(\int_{V_{0}} \delta \varepsilon^{T} S d V_{0}\right)-\int_{V_{0}} \dot{b}_{0}^{T} \delta u d V_{0}-\int_{A_{0}} \dot{t}_{0}^{T} \delta u d A_{0}
$$

Se forem considerados apenas carregamentos conservativos, $\dot{b}_{0}$ e $\dot{t}_{0}$ são nulos e a expressão anterior pode ser colocada na seguinte forma, já considerando-se a interpolação do campo de deslocamentos:

$$
\delta q^{T} \dot{r}=\delta q^{T} K_{T} \dot{q}
$$

onde:

$$
\frac{d}{d t} \int_{V_{0}} S^{T} \delta \varepsilon d V_{0}=\delta q^{T} K_{T} \dot{q}
$$

Sabendo-se que:

$$
\frac{d}{d t} \int_{V_{0}} S^{T} \delta \varepsilon d V_{0}=\int_{V_{0}} \delta \varepsilon^{T} \dot{S} d V_{0}+\int_{V_{0}} \delta \dot{\varepsilon}^{T}|\hat{S}| d V_{0}
$$


e efetuando-se as operações indicadas, a matriz de rigidez tangente para um elemento finito de treliça plana resulta de:

$$
\begin{aligned}
& K_{T}=\int_{V_{0}} B^{T} D_{11} B d V_{0}+\int_{V_{0}} G^{T} S_{11} G d V_{0}= \\
& =\int_{V_{0}}\left(B_{0}^{T} E B_{0}+B_{0}^{T} E B_{L}+B_{L}^{T} E B_{0}+B_{L}^{T} E B_{L}\right) d V_{0}+\int_{V_{0}} G^{T} S_{11} G d V_{0}
\end{aligned}
$$

Neste caso, em que se consideram pequenas as deformações, tem-se $D_{11}=E$ e $S_{11}=E \varepsilon_{11}$. A expressão (4.106) pode ser também escrita na forma:

$$
K_{T}=K_{0}+K_{L}+K_{\sigma},
$$

sendo cada parcela dada por:

$$
\begin{aligned}
& K_{0}=\int_{V_{0}} B_{0}^{T} E B_{0} d V_{0} \\
& K_{L}=\int_{V_{0}}\left(B_{0}^{T} E B_{L}+B_{L}^{T} E B_{0}+B_{L}^{T} E B_{L}\right) d V_{0} \\
& K_{\sigma}=\int_{V_{0}} G^{T} S_{11} G d V_{0}
\end{aligned}
$$

$K_{O}$ é a matriz de rigidez elástica linear, $K_{L}$ é a matriz de correção das coordenadas e $K_{\sigma}$ é a matriz de rigidez geométrica que é função do nível de solicitação axial das barras.

Realizando-se as operações indicadas em cada parcela da matriz de rigidez tangente, chega-se às seguintes matrizes:

$$
\begin{gathered}
K_{0}=\frac{E A_{0}}{L_{0}}\left[\begin{array}{cccc}
1 & 0 & -1 & 0 \\
0 & 0 & 0 & 0 \\
-1 & 0 & 1 & 0 \\
0 & 0 & 0 & 0
\end{array}\right] \\
K_{\sigma}=\frac{N}{L_{0}}\left[\begin{array}{cccc}
1 & 0 & -1 & 0 \\
0 & 1 & 0 & -1 \\
-1 & 0 & 1 & 0 \\
0 & -1 & 0 & 1
\end{array}\right]
\end{gathered}
$$




$$
\begin{gathered}
K_{L}=\frac{E A_{0}}{L_{0}^{2}}\left[\begin{array}{cccc}
2 \hat{U}_{1} & \hat{U}_{2} & -2 \hat{U}_{1} & -\hat{U}_{2} \\
\hat{U}_{2} & 0 & -\hat{U}_{2} & 0 \\
-2 \hat{U}_{1} & -\hat{U}_{2} & 2 \hat{U}_{1} & \hat{U}_{2} \\
-\hat{U}_{2} & 0 & \hat{U}_{2} & 0
\end{array}\right]+ \\
\frac{E A_{0}}{L_{0}^{3}}\left[\begin{array}{cccc}
\left(\hat{U}_{1}\right)^{2} & \hat{U}_{1} \hat{U}_{2} & -\left(\hat{U}_{1}\right)^{2} & -\hat{U}_{1} \hat{U}_{2} \\
\hat{U}_{1} \hat{U}_{2} & \left(\hat{U}_{2}\right)^{2} & -\hat{U}_{1} \hat{U}_{2} & -\left(\hat{U}_{2}\right)^{2} \\
-\left(\hat{U}_{1}\right)^{2} & -\hat{U}_{1} \hat{U}_{2} & \left(\hat{U}_{1}\right)^{2} & \hat{U}_{1} \hat{U}_{2} \\
-\hat{U}_{1} \hat{U}_{2} & -\left(\hat{U}_{2}\right)^{2} & \hat{U}_{1} \hat{U}_{2} & \left(\hat{U}_{2}\right)^{2}
\end{array}\right],
\end{gathered}
$$

onde: $\hat{U}_{1}=\left(q_{3}-q_{1}\right)$ e $\hat{U}_{2}=\left(q_{4}-q_{2}\right)$ 


\section{CAPÍTULO 5 - EXEMPLOS DE APLICAÇÕES}

Tanto os algoritmos como a modelação para estruturas, estudados anteriormente, foram implementadas num código de cálculo, que permite processar exemplos para verificar a eficiência e consistência de cada método. Os exemplos apresentados a seguir tem seus respectivos relatórios de dados e saída de resultados apresentados no Anexo.

\section{1 - Exemplo de Viga Contínua}

A estrutura considerada neste exemplo é uma viga contínua com restrição unilateral ao deslocamento vertical nos apoios. Os tramos possuem comprimento de $6,00 \mathrm{~m}$ e a seção transversal tem momento de inércia com relação ao eixo de flexão igual a $0,0027 \mathrm{~m}^{4}$, o material empregado possui módulo de elasticidade $\mathrm{E}=3,0 \mathrm{E}+7 \mathrm{kN} / \mathrm{m}^{2}$. $\mathrm{O}$ carregamento é constituído por uma carga vertical concentrada de $1200 \mathrm{kN}$ aplicada ao nó 6 da discretização e por uma carga uniformemente distribuída de $150 \mathrm{kN} / \mathrm{m}$ aplicada desde o nó 1 até o nó 3. Há uma folga de 0,01m entre o eixo e o nó 3, e de $0,02 \mathrm{~m}$ no nó 9 , vide figura (5.1):

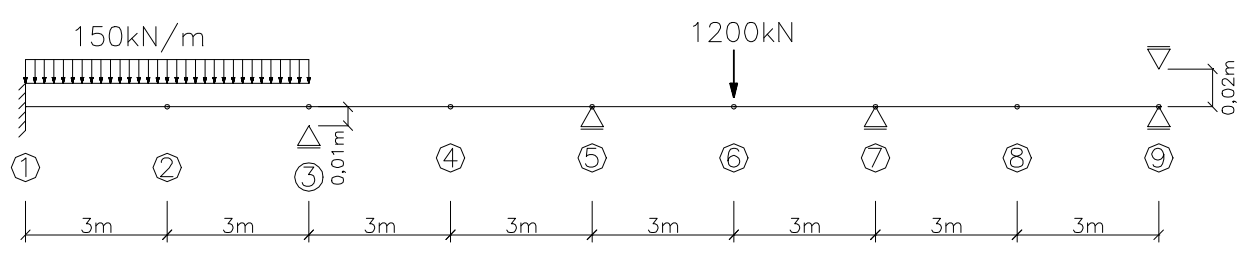


A tabela (5.1) mostra o desempenho dos métodos de otimização.

TABELA 5.1 - Comparação dos métodos para viga contínua.

\begin{tabular}{|l|c|c|}
\hline \multirow{2}{*}{ Método utilizado: } & \multicolumn{2}{|c|}{ Número de iterações } \\
\cline { 2 - 3 } & Busca exata & Busca aproximada \\
\hline Método do Gradiente & 130 & 408 \\
\hline Método de Newton & 4 & 17 \\
\hline Método de Quase-Newton & 16 & 39 (relaxação = 1) \\
\hline Método de Gauss-Seidel & \multicolumn{2}{|c|}{} \\
\hline
\end{tabular}

Os métodos do Gradiente, Newton e Gauss-Seidel convergiram para a mesma solução, enquanto que o de Quase-Newton apresentou uma diferença média em relação aos demais métodos de $16 \%$ com referência a deslocamentos e de $21 \%$ em reações. Note que o método de Newton apresentou o menor número de iterações para convergir, sendo assim o mais eficiente para este exemplo. A figura (5.2) ilustra a elástica obtida.

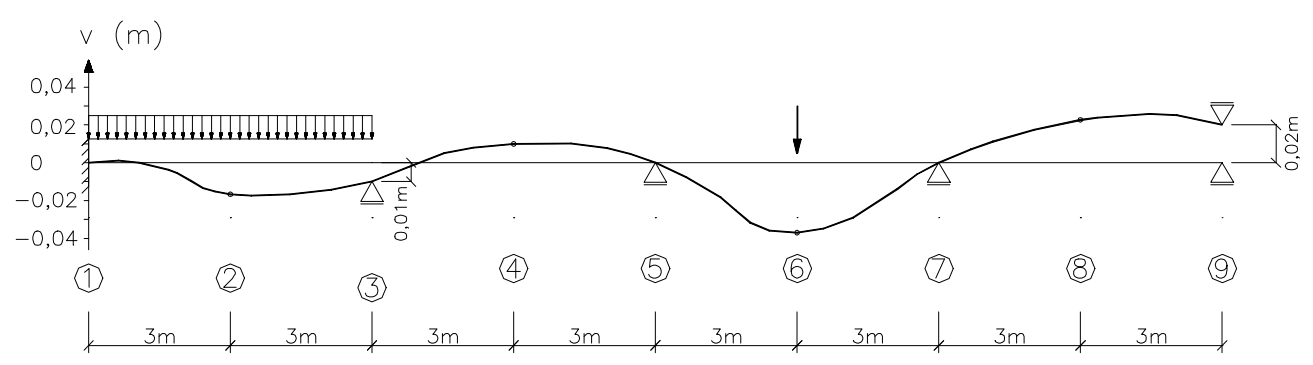

FIGURA 5.2 - Aspecto final da linha elástica da viga

O deslocamento vertical final do nó 6 para os métodos que convergiram para a mesma solução foi de $-3.6976 \mathrm{~cm}$. O método do Gradiente que precisou de um maior número de iterações para convergir, como mostra a tabela (5.1), apresentou uma 
boa convergência no início de seu processo iterativo, diminuindo sua eficiência conforme se aproxima da solução ótima. Isso já era de se esperar uma vez que se adotou uma tolerância baixa (tol $=10^{-6}$ ) e, devido ao fato do método do Gradiente desenvolver por Taylor o funcional $\pi$ até a $1^{\underline{a}}$ ordem, obtendo-se assim uma aproximação linear para um funcional quadrático.

A tabela (5.2) exemplifica a convergência do deslocamento vertical do nó 6 para o método do Gradiente utilizando-se a Busca exata.

TABELA 5.2 - Convergência do método do Gradiente.

\begin{tabular}{|c|c|c|}
\hline Iteração & $\begin{array}{c}\text { Deslocamento vertical do nó } \mathbf{6}\left(\boldsymbol{\delta}_{\mathbf{v}}\right)_{\mathbf{6}} \\
(\mathbf{c m})\end{array}$ & $\begin{array}{c}\text { Fator de convergência } \\
\left(\boldsymbol{\delta}_{\mathbf{v}}\right)_{\mathbf{6}} /\left[\left(\boldsymbol{\delta}_{\mathbf{v}}\right)_{\mathbf{6}} \text { final }\right.\end{array}$ \\
\hline 10 & $-2,9335$ & 0,793 \\
\hline 25 & $-3,4520$ & 0,933 \\
\hline 50 & $-3,6228$ & 0,979 \\
\hline 75 & $-3,6870$ & 0,997 \\
\hline 100 & $-3,6966$ & 0,9997 \\
\hline 125 & $-3,6975$ & 0,99997 \\
\hline 130 & $-3,6976$ & 1,000 \\
\hline
\end{tabular}

O contato unilateral que acontece nos nós 3 e 9, devido à deformação da viga proporcionada pelo carregamento, como mostra a figura (5.2), são restrições ao deslocamento vertical que foram atendidas implicitamente pelos métodos de otimização.

\section{2 - Exemplo de Pórtico Plano}

A estrutura considerada neste exemplo é um pórtico plano composto de duas barras, sendo uma de 4,00m e outra de 7,00m, discretizada em 5 elementos conforme figura (5.3). As barras que formam o pórtico têm área de seção transversal igual a $3,00 \mathrm{~cm}^{2}$ e momento de inércia de $1,00 \mathrm{~cm}^{4}$; o material empregado possui módulo de elasticidade $\mathrm{E}=10.000 \mathrm{kN} / \mathrm{cm}^{2}$. O nó 4 possui uma folga horizontal com relação ao apoio de $0,07 \mathrm{~cm}$. 


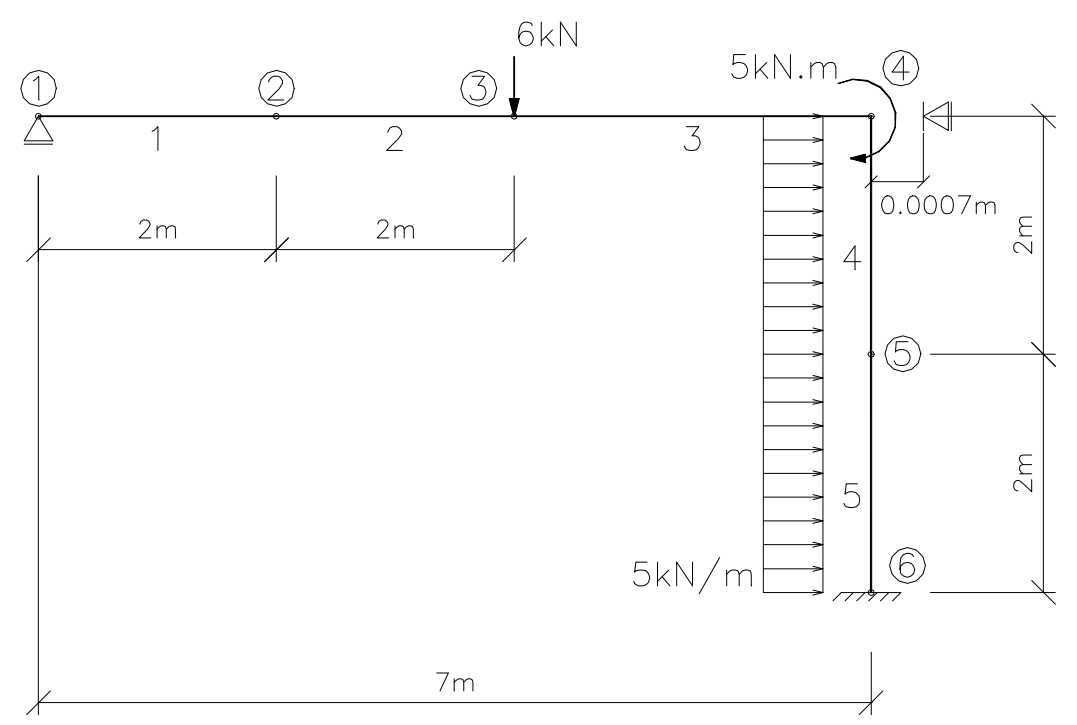

FIGURA 5.3 - Pórtico plano

A tabela (5.3) mostra o deslocamento vertical para baixo do nó 4 , e o desempenho de cada método.

TABELA 5.3 - Comparação dos métodos para pórtico plano.

\begin{tabular}{|l|c|c|c|c|}
\hline \multirow{2}{*}{ Método utilizado: } & \multicolumn{4}{|c|}{ Deslocamento vertical do nó 4 (cm) } \\
\cline { 2 - 5 } & Busca Exata & Iterações & Busca Aproximada & Iterações \\
\hline Gradiente & $-0,05657$ & 17 & $-0,05658$ & 23 \\
\hline Newton & $-0,05658$ & 3 & $-0,05658$ & 3 \\
\hline Quase-Newton & $-0,05658$ & 5 & $-0,05658$ & 15 \\
\hline Gauss-Seidel & $-0,05658$ & \multicolumn{3}{|c|}{ Relaxação $=1.00$} \\
\hline
\end{tabular}




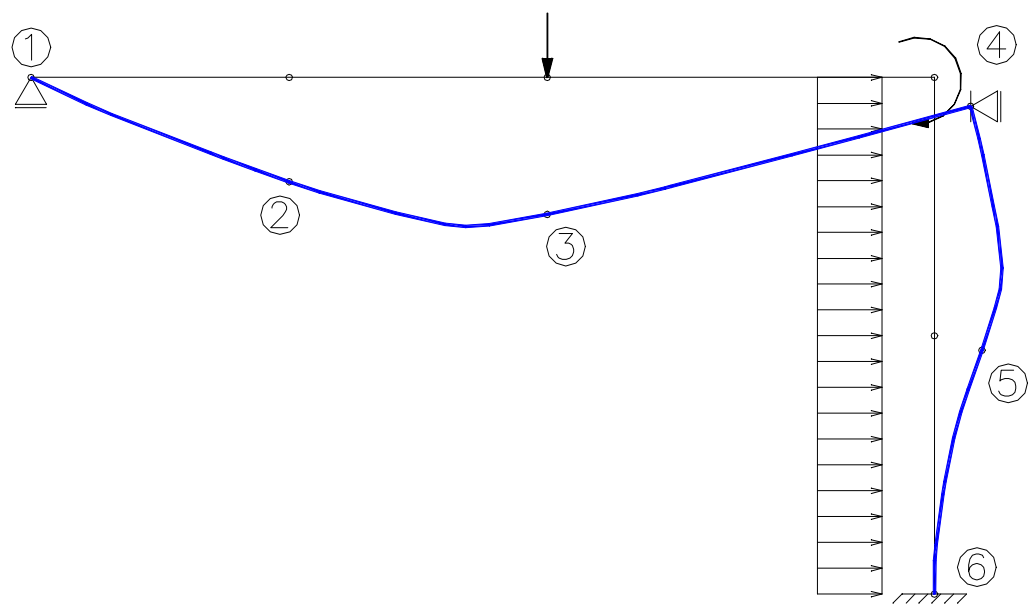

FIGURA 5.4 - Linha elástica do pórtico plano

Desta vez os quatro métodos convergiram para a mesma solução e, novamente, o método de Newton foi o que convergiu com o menor número de iterações. Nesse exemplo, a primeira iteração do método de Newton conduz o vetor de deslocamentos para a face que contém a restrição do tipo contato unilateral (deslocamento horizontal do nó 4), na segunda iteração o método converge para a solução ótima, a terceira iteração confirma a solução anterior e, através da norma infinita entre os dois vetores de deslocamentos o programa encerra o processo iterativo. Como o método de Newton trabalha com uma aproximação quadrática da função a ser minimizada e também como neste caso, o funcional aproximado da energia total é quadrático, esse método mostra-se muito eficiente.

\section{3 - Exemplo de Treliça Espacial}

A estrutura considerada neste exemplo é uma treliça espacial de base quadrada, com lado igual a $4,00 \mathrm{~m}$ e altura total de $20,00 \mathrm{~m}$. As barras que formam as colunas têm área de seção transversal igual a $35,00 \mathrm{~cm}^{2}$ e as demais $7,50 \mathrm{~cm}^{2}$; o material empregado possui módulo de elasticidade $\mathrm{E}=21.000 \mathrm{kN} / \mathrm{cm}^{2}$. A treliça é simétrica. $\mathrm{O}$ carregamento total aplicado é formado por uma força vertical de $3000 \mathrm{kN}$ e outra horizontal de $400 \mathrm{kN}$ no ponto 9 e forças horizontais de $300 \mathrm{kN}$ nos pontos 6 e 8 . O nó 
9 possui uma folga horizontal em relação ao apoio de $20 \mathrm{~cm}$ (vínculo unilateral) e os nós 5 e 7 possuem uma folga horizontal de $4 \mathrm{~cm}$, como ilustra a figura (5.5).

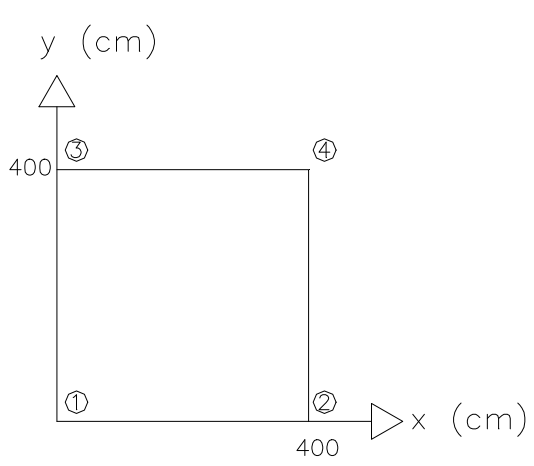

a) Planta da base

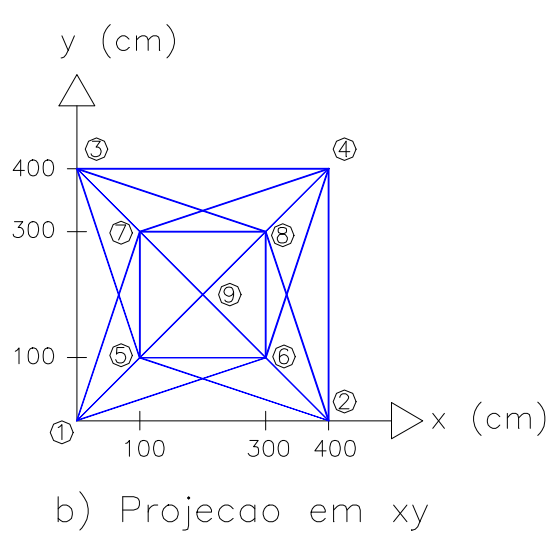

b) Projecao em xy

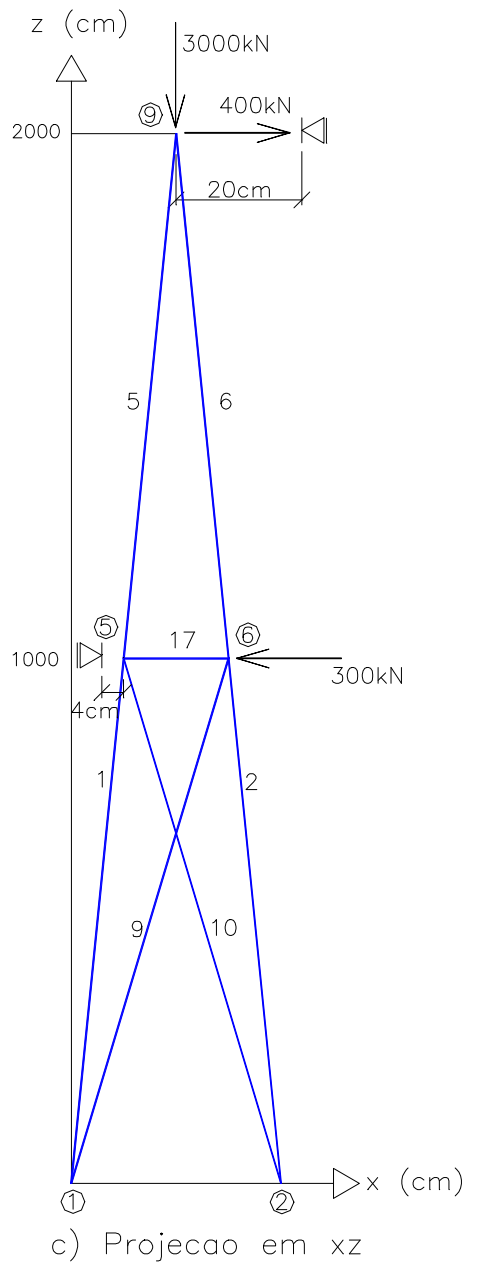

FIGURA 5.5 - Treliça espacial de base quadrada

Não foi considerado neste exemplo a não-linearidade geométrica, portanto trata-se de uma análise linear da treliça espacial, que possui restrições ao deslocamentos horizontais dos nós 5,7 e 9. A tabela (5.4) mostra o deslocamento 
horizontal dos nós 5/7 e 9, desconsiderando-se as restrições de folga horizontal aos apoios dos nós 5/7 e 9. Nesse exemplo foi utilizado a busca unidimensional Exata.

TABELA 5.4 - Treliça espacial sem o problema de contato unilateral (minimização irrestrita)

\begin{tabular}{|l|c|c|c|}
\hline \multirow{2}{*}{ Método utilizado: } & \multicolumn{3}{|c|}{ Deslocamento horizontal (cm) } \\
\cline { 2 - 4 } & Nós 5/7 & Nó 9 & Iterações \\
\hline Gradiente & $-4,9569$ & 21,3847 & 1799 \\
\hline Newton & $-4,9569$ & 21,3847 & 2 \\
\hline Quase-Newton & $-4,9569$ & 21,3847 & 10 \\
\hline Gauss-Seidel & $-4,9569$ & 21,3847 & 318 \\
\hline
\end{tabular}

No método de Gauss-Seidel, o valor da relaxação utilizado foi igual a 1.

Novamente se nota que o método do Gradiente convergiu com um elevado número de iterações, devido aos mesmos motivos verificados na convergência do exemplo (5.1). Além disso, o maior número de deslocamentos incógnitos do funcional da energia para essa treliça espacial, contribuiu significativamente para que o método realizasse um grande número de iterações muito próximo a solução ótima.

Considerando-se o problema de contato unilateral conforme ilustra a figura (5.5), têm-se:

TABELA 5.5 - Treliça espacial com problema de contato unilateral (minimização restrita)

\begin{tabular}{|l|c|c|c|}
\hline \multirow{2}{*}{ Método utilizado: } & \multicolumn{3}{|c|}{ Deslocamento horizontal (cm) } \\
\cline { 2 - 4 } & Nós 5/7 & Nó 9 & Iterações \\
\hline Gradiente & $-4,0000$ & 20,0000 & 275 \\
\hline Newton & $-4,0000$ & 17,2565 & 3 \\
\hline Quase-Newton & $-4,0000$ & 18,8182 & 10 \\
\hline
\end{tabular}

A configuração final da linha elástica pelo método de Newton é ilustrada na figura (5.6): 


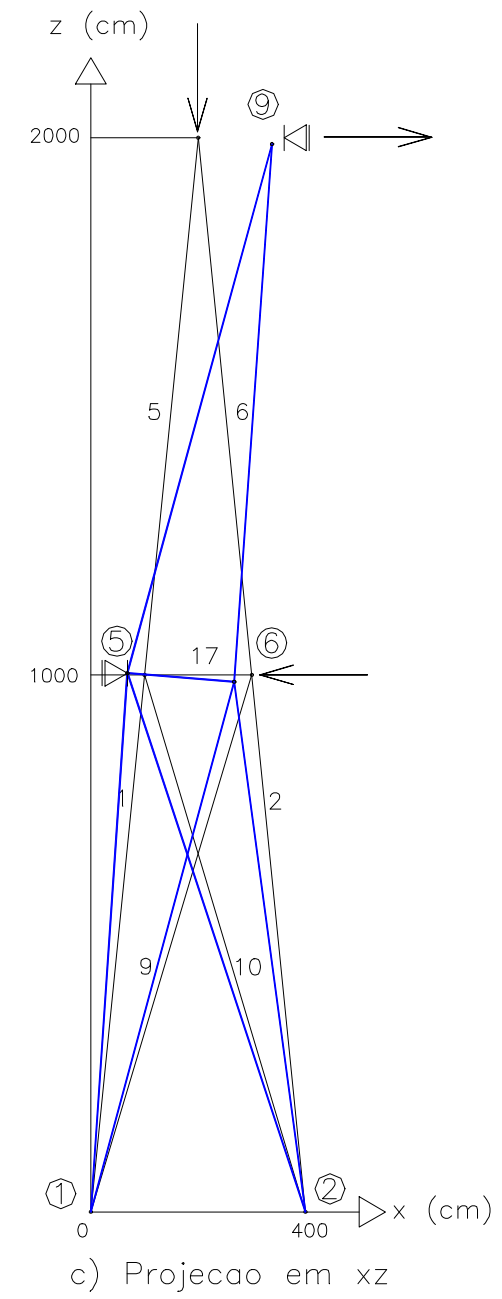

FIGURA 5.6 - Configuração final da elástica da treliça espacial

Note que os deslocamentos dos nós da figura (5.6) foram aumentados em oito vezes em relação a escala do desenho para uma melhor visualização. Os valores exatos desses deslocamentos podem ser encontrados no arquivo de saída de dados e resultados do exemplo 3 do Anexo.

Os três métodos convergiram para soluções diferentes, porém o método do Gradiente além de precisar de um número maior de iterações, obteve um deslocamento horizontal para o nó 9 de $20 \mathrm{~cm}$, utilizando-se assim toda a folga horizontal em relação ao apoio. Dessa forma, realizou-se um contato entre o nó 9 e o apoio, o qual não se observou para os métodos de Newton e Quase-Newton, cujos deslocamentos horizontais do nó 9 foram inferior a $20 \mathrm{~cm}$. 
Provavelmente, esse fato ocorre devido a melhor eficiência dos dois últimos métodos, uma vez que estes foram combinados com a estratégia de conjuntos ativos justamente para os problemas de minimização com variáveis canalizadas, proporcionando aos métodos a possibilidade de efetuar uma minimização na face.

\section{4 - Exemplo de Viga Treliçada bi-apoiada}

Este exemplo está proposto em BANDEIRA (1987), tratando-se de uma viga plana simétrica em treliça (ver figura 5.7), analisada considerando-se a não linearidade geométrica. A estrutura tem vão de $6,00 \mathrm{~m}$, altura de $1,50 \mathrm{~m}$ e distância entre cargas de 2,00m. As barras com numeração de 11 a 31 têm área de seção transversal igual a $3,77 \mathrm{~cm}^{2}$ e as demais $4,88 \mathrm{~cm}^{2}$; o material empregado possui módulo de elasticidade longitudinal $\mathrm{E}=2,1 \mathrm{E}+7 \mathrm{ton} / \mathrm{m}^{2}$. O carregamento total consiste de duas forças verticais de 2,20 ton aplicados nos nós 15 e 19. Os nós 2 a 10 têm restrição de contato unilateral, podendo se deslocar livremente na vertical até $-0,30 \mathrm{~m}$.

Neste caso o material segue uma relação constitutiva elástica não-linear com as seguintes características:

$$
\begin{array}{ll}
\sigma=0,01 \varepsilon-19603,91986 & ; \varepsilon<-0,014 \\
\sigma=E\left(0,1 \varepsilon-170 \varepsilon^{3}\right) & ;-0,014 \leq \varepsilon \leq 0,014 \\
\sigma=0,01 \varepsilon+19603,91986 & ; \varepsilon>0,014
\end{array}
$$

No método de Newton utilizou-se a matriz de rigidez tangente a cada iteração e o procedimento numérico de otimização a cada passo de carga. 


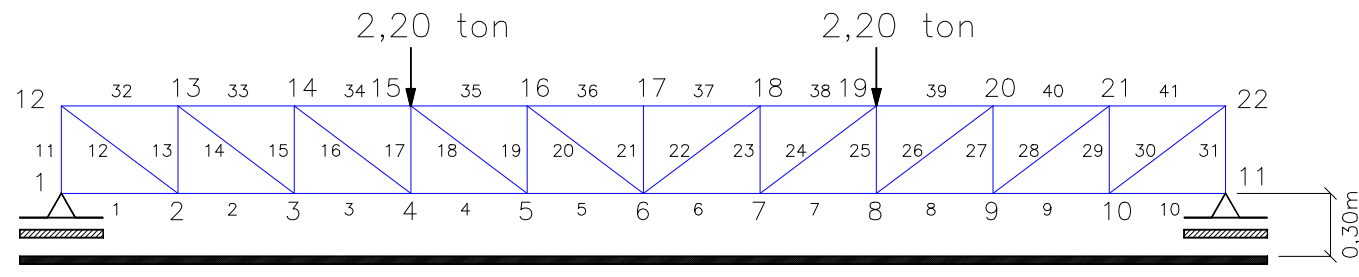

FIGURA 5.7 - Viga simétrica bi-apoiada - Problema de contato

Entre os métodos testados no trabalho, o Método de Newton combinado com a estratégia dos conjuntos ativos convergiu com maior eficiência para a solução apresentada no trabalho de BANDEIRA (1987). Na análise o carregamento total foi aplicado em 50 passos, sendo que no passo de carga 25 o nó 6 atingiu seu contato unilateral, ao se deslocar -0,30m na direção vertical. No passo de carga 49 os nós 5 e 7 também atingiram a restrição de contato. A configuração de equilíbrio da treliça é ilustrada na figura (5.8). O relatório com a saída de resultados do último passo de carga se encontra no anexo. 


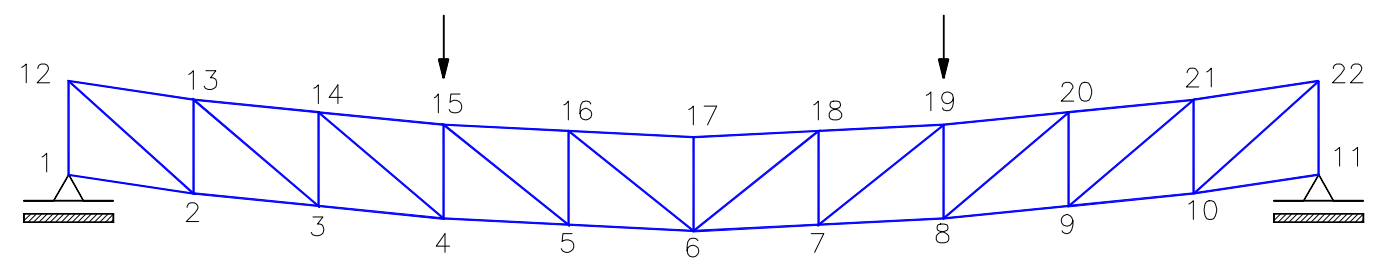

FIGURA 5.8 - Linha elástica da viga simétrica bi-apoiada

Os métodos de Newton e Quase-Newton combinados com a estratégia dos conjuntos ativos e do Gradiente obtiveram os mesmos resultados, porém o número médio de iterações para cada passo de carga foi de 4, 650 e 1230 respectivamente, o que demonstra a melhor eficiência do método de Newton em relação aos demais.

A tabela (5.6) mostra o deslocamento vertical dos nós 4 e 6 ao longo do carregamento.

TABELA 5.6 - Deslocamento vertical segundo o carregamento.

\begin{tabular}{|c|c|c|}
\hline $\begin{array}{l}\text { Porcentagem do } \\
\text { Carregamento }\end{array}$ & $\begin{array}{l}\text { Deslocamento vertical } \\
\text { do nó } \mathbf{4}(\mathbf{m})\end{array}$ & $\begin{array}{l}\text { Deslocamento vertical } \\
\text { do nó 6 (m) }\end{array}$ \\
\hline $20 \%$ & $-0,1135$ & $-0,1182$ \\
\hline $40 \%$ & $-0,2310$ & $-0,2406$ \\
\hline $60 \%$ & $-0,2907$ & $-0,3000$ \\
\hline $80 \%$ & $-0,2957$ & $-0,3000$ \\
\hline $100 \%$ & $-0,3000$ & $-0,3000$ \\
\hline
\end{tabular}




\section{CAPÍTULO 6 - CONSIDERAÇÕES FINAIS E CONCLUSÕES}

Este trabalho faz uso dos Métodos de Otimização como ferramentas consistentes e muito úteis para a análise do comportamento não-linear de estruturas.

Naturalmente, os algoritmos de otimização estudados aqui podem ser estendidos para outros problemas de engenharia. No entanto, particularizando-se para o caso de estruturas cuja não-linearidade do comportamento decorre das condições de vinculação, esses algoritmos proporcionam uma estratégia numérica de resolução de equações não-lineares.

Por outro lado, o Método da Energia aplicado à modelagem clássica de estruturas, considerando-se um regime de pequenos deslocamentos e resposta elástica linear do material, fundamenta-se no equacionamento da energia total envolvida no sistema durante o processo de carregamento e deformação, sendo que o mínimo da energia total corresponde à situação de equilíbrio. Na forma clássica a análise de problemas estruturais apresentam uma minimização irrestrita.

Inicialmente, foram estudados os métodos de resolução de problemas de minimização irrestrita. Entre os métodos do Gradiente, Quase-Newton e Newton, este último se destaca pela simplicidade e razão de convergência. Basicamente, a principal diferença entre os métodos é a forma como eles tomam a direção de descida na busca de um ponto de mínimo local ou global. Nessa fase ainda foram estudados procedimentos para avaliar o tamanho do passo na direção de descida, através das buscas unidimensionais exata, empregada apenas para funções quadráticas e, aproximada, através da "Regra de Armijo".

Já os problemas de contato em estruturas reticulares impõem restrições sobre o problema da minimização da energia. Devido a essa motivação, iniciou-se o estudo e implementação dos algoritmos de minimização restritos.

O método de Newton, que foi o mais eficiente para otimização irrestrita, não apresentou resultados satisfatórios na minimização com variáveis canalizadas, pois 
quando a solução atingia uma face da região de factibilidade não havia um critério de como se caminhar na direção de descida, mantendo-se sobre ela.

Este problema foi resolvido ao se combinar a estratégia dos conjuntos ativos ao método de Newton. Essa estratégia consiste em estabelecer que quando a solução atinge uma face, as restrições que alcançaram seu limite inferior ou superior tornam-se ativas (fixas), e promove-se uma minimização irrestrita nesta face. Assim o método de Newton combinado com essa estratégia se tornou eficiente para se analisar estruturas com restrições aos deslocamentos do tipo unilateral.

Os métodos de otimização estudados neste trabalho foram aplicados na análise de estruturas reticulares como vigas, pórticos e treliças espaciais considerandose vinculações do tipo unilateral. O caso particular das treliças planas foi estudado com dupla não-linearidade, incluindo-se a consideração dos grandes deslocamentos. Todos os exemplos foram processados a partir de um único programa que possui a opção de uso de cada um dos algoritmos de minimização.

O exemplo 1 ilustrou a análise do comportamento de uma viga contínua, com restrições de vínculo do tipo unilateral, comparando-se a eficiência dos vários métodos de otimização e dos procedimentos de busca. O método de Newton combinado com a estratégia dos conjuntos ativos obteve o melhor desempenho. $\mathrm{O}$ aspecto final da linha elástica reforça bem que a minimização foi realizada satisfazendo contatos unilaterais.

O exemplo 2 ilustrou a análise do comportamento de um pórtico plano, com vínculo unilateral disposto num de seus nós. O método de Newton combinado com a estratégia dos conjuntos ativos obteve, novamente, o melhor desempenho, porém os demais métodos também foram muito satisfatórios.

O exemplo 3 procurou dar ênfase ao fato de que quanto maior o número de coordenadas empregadas na discretização da estrutura, mais se acentua a diferença de desempenho entre os métodos de minimização estudados. $\mathrm{Na}$ análise do comportamento da treliça espacial, os métodos de Newton e Quase-Newton combinados com a estratégia dos conjuntos ativos tiveram um número de iterações muito menor do que a do Gradiente, além de uma solução diferenciada.

Finalmente, o exemplo 4 ilustrou a análise de uma treliça plana com nãolinearidades dos tipos geométrica e de contatos unilaterais. Neste caso apenas os métodos de Newton e Quase-Newton são indicados. Além disso, o método de QuaseNewton mostra-se uma alternativa muito interessante pois fornece uma regra de 
atualização da matriz de rigidez absolutamente essencial para a redução dos erros induzidos no procedimento incremental. Para esse tipo de problema, a quantidade de passos de carga influem na convergência para a solução exata. Todavia, verificou-se que para o caso de não-linearidade geométrica em treliça plana sem restrições a deslocamentos, com apenas um passo de carga o problema já convergia para a solução esperada.

De um modo geral, pode-se afirmar que o objetivo proposto inicialmente foi alcançado com êxito, destacando-se a combinação original do método de Newton com uma estratégia dos conjuntos ativos para a análise não-linear de estruturas.

Apesar do campo de aplicações dos métodos de otimização na análise de estruturas ser muito vasto, como sugestão para continuidade deste trabalho destaca-se a extensão do estudo para análises planas e o emprego de outros métodos do tipo QuaseNewton, que fornecem regras de atualização da matriz de rigidez da estrutura. 


\section{BIBLIOGRAFIA}

ASSAN, A.E. (1996) Métodos Energéticos e Análise Estrutural. Campinas, Editora da UNICAMP.

BANDEIRA, A.A. (1987) Uma Introdução a Problemas de Contato. São Paulo. Dissertação (Mestrado) - Escola Politécnica da Universidade de São Paulo.

BAZARAA, M.S.; SHETTY C.M. (1979) Nonlinear Programming, Theory and Algorithims. New York, John Wiley.

BREBBIA, C.A.; CONNOR, J.J. (9175) Metodo de los Elementos Finitos en la Ingenieria Civil. Centro de Perfeccionamento Profesional y Empresarial, Colegio de Ingenieros de Caminos, Canales y Puertos, Madrid.

FEIJOÓ, R.A.; BARBOSA, H.J.C. (1983) Análisis Estática de Vigas Elásticas em Apoyos Unilaterales. Rio de Janeiro, L.N.C.C.

GERE, J.M., TIMOSHENKO S.P. (1984) Mecânica dos Sólidos. Rio de Janeiro, Ao Livro Técnico - S.A., Volume II.

GERE, J.M.; WEAVER JR.W. (1981) Análise de Estruturas Reticuladas. Rio de Janeiro, Guanabara Dois.

GOMES, F.A.M. (1995) Minimização de funções quadráticas com álgebra linear adaptativa e aplicações. Campinas. Tese (Doutorado) - Instituto de Matemática, Estatística e Computação Científica, Universidade Estadual de Campinas.

HUMES, A.F.P.C. et al. (1984) Noções de cálculo numérico. São Paulo, McGrawHill.

LAIER, J.E.; BARREIRO, J.C. (1983) Complementos de Resistência dos Materiais. São Carlos. Escola de Engenharia de São Carlos, Universidade de São Paulo, 208p. (Apostila) 
LAZARIN, C.; FRANCO, N.M.B. (1991) Tópicos de Cálculo Numérico. São Carlos. Instituto de Ciências Matemáticas de São Carlos, Universidade de São Paulo, 145p. (Apostila)

LUENBERGER, D.G. (1984) Linear and Nonlinear Programming. Adilson, Wesley.

PAULA, C.F. (1997). Estudo das Descrições Lagrangiana e Euleriana na Análise Não-Linear Geométrica com o Emprego do Método dos Elementos Finitos. São Carlos. Dissertação (Mestrado) - Escola de Engenharia de São Carlos, Universidade de São Paulo.

PROENÇA, S.P.B. Análise não-linear de estruturas. São Carlos. Escola de Engenharia de São Carlos, Universidade de São Paulo, 127p. (Notas de Aula)

PROENÇA, S.P.B.; SAVASSI, W; MUNAIAR NETO, J. Aplicação do Procedimento Iterativo de Gaus-Seidel na Automatização do Cálculo de Vigas Contínuas. São Carlos. Escola de Engenharia de São Carlos, Universidade de São Paulo. (Apostila)

RUBERT, J.B. (1993) Estudo do Desempenho de Algoritmos Numéricos na Solução de Sistemas não-lineares de Estruturas Formadas por Barras de Treliça. São Carlos. Dissertação (Mestrado) - Escola de Engenharia de São Carlos, Universidade de São Paulo.

SCHIEL, F. (1984) Introdução à Resistência de Materiais. São Paulo, Harbra Harper \& Row do Brasil, 392p.

SOUZA, J.C.A.O.; ANTUNES, H.M.C.C. (1992) Processos gerais da hiperestática clássica. São Carlos. Escola de Engenharia de São Carlos, Universidade de São Paulo, 346p.

SOUZA, J.C.A.O.; ANTUNES, H.M.C.C. (1993) Técnicas Computacionais na

Estática das Estruturas. São Carlos. Escola de Engenharia de São Carlos, Universidade de São Paulo, 106p. (Apostila)

TIMOSHENKO, S.P. (1967) Resistência dos Materiais. Rio de Janeiro, Ao Livro Técnico - S.A., Volume I.

WASHIZU, K. (1968) Variational methods in elasticity and platicity. Pergamon Press. 
ZIENKIEWICZ, O.C.; TAYLOR, R.L. (1991) The Finite Element Method. São Paulo, McGraw-Hill, Volume I e II. 


\section{ANEXO}

\section{Arquivos de entrada e saída para o exemplo 1:}

\section{Arquivo de Entrada de Dados:}

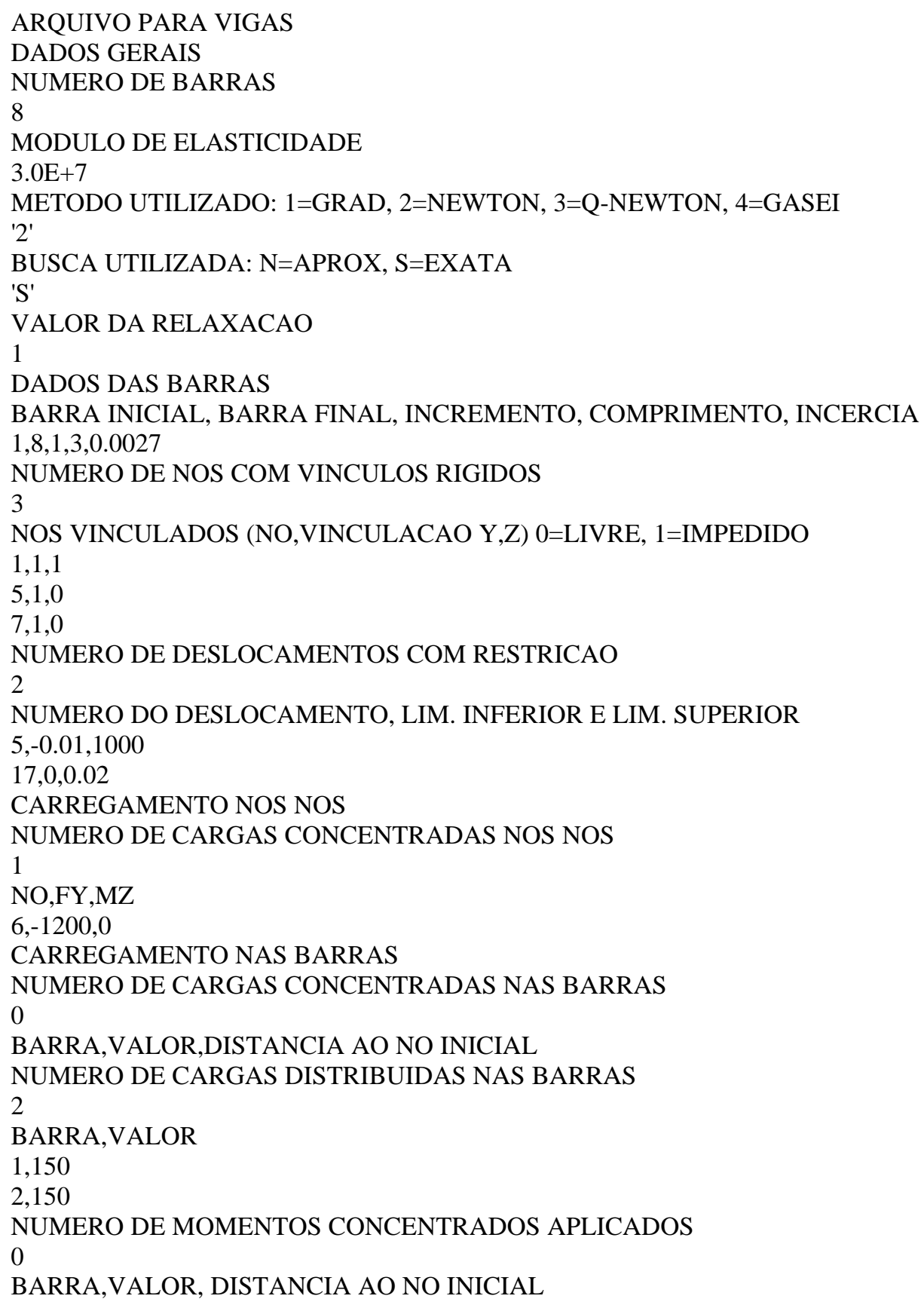




\section{Arquivo de Saída de Dados:}

PROGRAMA PARA VIGA

Numero de nós $=9$

Numero de Barras $=8$

Modulo de Elasticidade $=.3000 \mathrm{E}+08$

A minimizacao foi feita pelo Metodo de Newton

A minimizacao utilizou a busca unidimens. Exata

Informacoes sobre os Nos: restr. a desloc. e giros

No lim. inf. lim. sup.

$\begin{array}{lll}1 & -.1000 \mathrm{E}+04 & .1000 \mathrm{E}+04\end{array}$

$1-.1000 \mathrm{E}+04 \quad .1000 \mathrm{E}+04$

$2-.1000 \mathrm{E}+04 \quad .1000 \mathrm{E}+04$

$2-.1000 \mathrm{E}+04 \quad .1000 \mathrm{E}+04$

$3 \quad-.1000 \mathrm{E}-01 \quad .1000 \mathrm{E}+04$

$3 \quad-.1000 \mathrm{E}+04 \quad .1000 \mathrm{E}+04$

$4-.1000 \mathrm{E}+04 \quad .1000 \mathrm{E}+04$

$4 \quad-.1000 \mathrm{E}+04 \quad .1000 \mathrm{E}+04$

$5 \quad-.1000 \mathrm{E}+04 \quad .1000 \mathrm{E}+04$

$\begin{array}{lll}5 & -.1000 \mathrm{E}+04 & .1000 \mathrm{E}+04\end{array}$

$6 \quad-.1000 \mathrm{E}+04 \quad .1000 \mathrm{E}+04$

$6 \quad-.1000 \mathrm{E}+04 \quad .1000 \mathrm{E}+04$

$\begin{array}{lll}7 & -.1000 \mathrm{E}+04 & .1000 \mathrm{E}+04\end{array}$

$7 \quad-.1000 \mathrm{E}+04 \quad .1000 \mathrm{E}+04$

$8 \quad-.1000 \mathrm{E}+04 \quad .1000 \mathrm{E}+04$

$8 \quad-.1000 \mathrm{E}+04 \quad .1000 \mathrm{E}+04$

$9 \quad .0000 \mathrm{E}+00 \quad .2000 \mathrm{E}-01$

$9-.1000 \mathrm{E}+04 \quad .1000 \mathrm{E}+04$

Informacoes sobre as Barras

Barra Mom Inercia Comprimento

$1 \quad .2700 \mathrm{E}-02 \quad .3000 \mathrm{E}+01$

$2 \quad .2700 \mathrm{E}-02 \quad .3000 \mathrm{E}+01$

$3 \quad .2700 \mathrm{E}-02 \quad .3000 \mathrm{E}+01$

$4 \quad .2700 \mathrm{E}-02 \quad .3000 \mathrm{E}+01$

$5 \quad .2700 \mathrm{E}-02 \quad .3000 \mathrm{E}+01$

$6 \quad .2700 \mathrm{E}-02 \quad .3000 \mathrm{E}+01$

$7 \quad .2700 \mathrm{E}-02 \quad .3000 \mathrm{E}+01$

$8 \quad .2700 \mathrm{E}-02 \quad .3000 \mathrm{E}+01$

Cargas Aplicadas aos Nos

No Forca Y Momento Z

$6 \quad-.1200 \mathrm{E}+04 \quad .0000 \mathrm{E}+00$

---Cargas nas Barras---

Cargas Uniformemente Distribuidas

Barra Valor

$1 \quad .1500 \mathrm{E}+03$

$2.1500 \mathrm{E}+03$ 

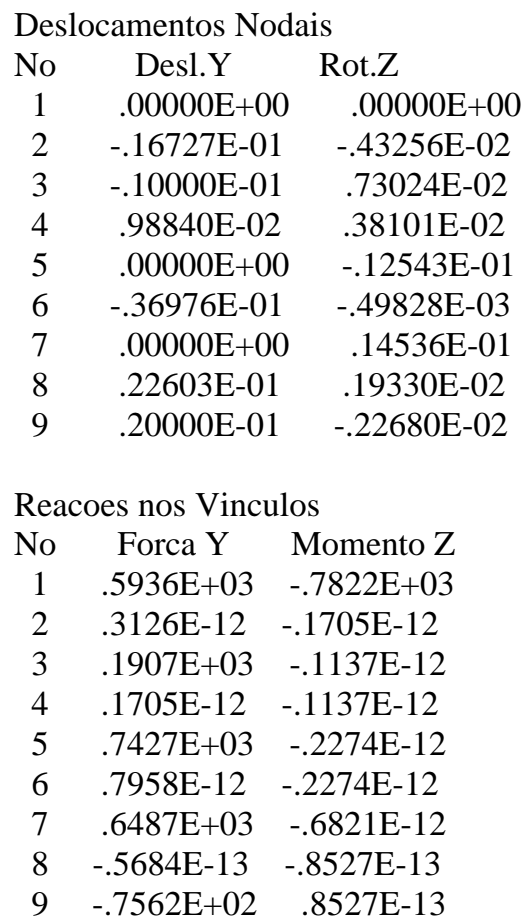

Esforcos nas Extremidades das barras

\begin{tabular}{crcrcr}
\multicolumn{5}{c}{ Inicio da Barra } & \multicolumn{3}{c}{ Fim da Barra } \\
Barras & FY & \multicolumn{1}{l}{ MZ } & FY & $M$ & \\
1 & $.5936 \mathrm{E}+03$ & $.7822 \mathrm{E}+03$ & $-.1436 \mathrm{E}+03$ & $.3236 \mathrm{E}+03$ \\
2 & $.1436 \mathrm{E}+03$ & $-.3236 \mathrm{E}+03$ & $.3064 \mathrm{E}+03$ & $.7933 \mathrm{E}+02$ \\
3 & $-.1157 \mathrm{E}+03$ & $-.7933 \mathrm{E}+02$ & $.1157 \mathrm{E}+03$ & $-.2679 \mathrm{E}+03$ \\
4 & $-.1157 \mathrm{E}+03$ & $.2679 \mathrm{E}+03$ & $.1157 \mathrm{E}+03$ & $-.6152 \mathrm{E}+03$ \\
5 & $.6269 \mathrm{E}+03$ & $.6152 \mathrm{E}+03$ & $-.6269 \mathrm{E}+03$ & $.1266 \mathrm{E}+04$ \\
6 & $-.5731 \mathrm{E}+03$ & $-.1266 \mathrm{E}+04$ & $.5731 \mathrm{E}+03$ & $-.4537 \mathrm{E}+03$ \\
7 & $.7562 \mathrm{E}+02$ & $.4537 \mathrm{E}+03$ & $-.7562 \mathrm{E}+02$ & $-.2269 \mathrm{E}+03$ \\
8 & $.7562 \mathrm{E}+02$ & $.2269 \mathrm{E}+03$ & $-.7562 \mathrm{E}+02$ & $-.8527 \mathrm{E}-13$
\end{tabular}

\section{Arquivos de entrada e saída para o exemplo 2:}

\section{Arquivo de Entrada de Dados:}

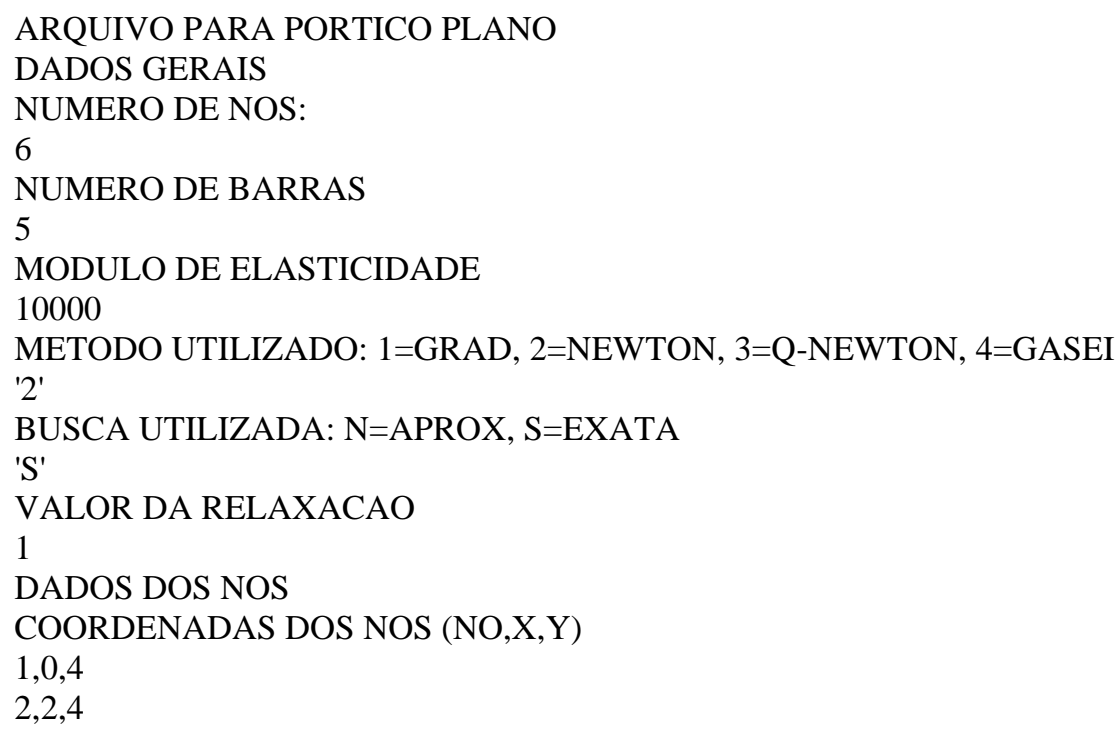


NUMERO DE NOS COM VINCULOS RIGIDOS

2

NOS VINCULADOS (NO,VINCULACAO X,Y,Z) 0=LIVRE, 1=IMPEDIDO

$1,1,1,0$

$6,1,1,1$

NUMERO DE DESLOCAMENTOS COM RESTRICAO

1

NUMERO DO DESLOCAMENTO, LIM. INFERIOR E LIM. SUPERIOR

$10,-1,0.0007$

DADOS DAS BARRAS

INCIDENCIA - BARRA, NO INICIAL, NO FINAL

$1,1,2$

$2,2,3$

$3,3,4$

$4,4,5$

$5,5,6$

CARACTERISTICAS GEOMETRICAS - B.INICIAL,B.FINAL,INCREMENTO,AX,IZ

$1,5,1,3,1$

CARREGAMENTO NOS NOS

NUMERO DE CARGAS CONCENTRADAS NOS NOS

2

NO,FX,FY,FZ

$3,0,-6,0$

$4,0,0,-5$

CARREGAMENTO NAS BARRAS

NUMERO DE CARGAS CONCENTRADAS NAS BARRAS

0

BARRA,DIRECAO(1,2,3),VALOR,DISTANCIA AO NO INICIAL

NUMERO DE CARGAS DISTRIBUIDAS NAS BARRAS

2

BARRA,DIRECAO(1,2,3),VALOR,DISTANCIA DO INICIO E DO FIM AO NO INICIAL

$4,2,5,0,2$

$5,2,5,0,2$

\section{Arquivo de Saída de Resultados:}

PROGRAMA PARA PORTCICO PLANO

$$
\begin{aligned}
& \text { Numero de nos }=6 \\
& \text { Numero de Barras }=5 \\
& \text { Modulo de Elasticidade }=.1000 \mathrm{E}+05 \\
& \text { A minimizacao foi feita pelo Metodo de Newton } \\
& \text { A minimizacao utilizou a busca unidimens. exata } \\
& \text { Informacoes sobre os Nos: restr. a desloc. e giros } \\
& \begin{array}{ccc}
\text { No } & \text { lim. inf. } & \text { lim. sup. } \\
1 & -.1000 \mathrm{E}+04 & .1000 \mathrm{E}+04 \\
1 & -.1000 \mathrm{E}+04 & .1000 \mathrm{E}+04 \\
1 & -.1000 \mathrm{E}+04 & .1000 \mathrm{E}+04 \\
2 & -.1000 \mathrm{E}+04 & .1000 \mathrm{E}+04 \\
2 & -.1000 \mathrm{E}+04 & .1000 \mathrm{E}+04 \\
2 & -.1000 \mathrm{E}+04 & .1000 \mathrm{E}+04 \\
3 & -.1000 \mathrm{E}+04 & .1000 \mathrm{E}+04 \\
3 & -.1000 \mathrm{E}+04 & .1000 \mathrm{E}+04 \\
3 & -.1000 \mathrm{E}+04 & .1000 \mathrm{E}+04 \\
4 & -.1000 \mathrm{E}+01 & .7000 \mathrm{E}-03 \\
4 & -.1000 \mathrm{E}+04 & .1000 \mathrm{E}+04
\end{array}
\end{aligned}
$$$$
\text { A minimizacao utilizou a busca unidimens. exata }
$$ 


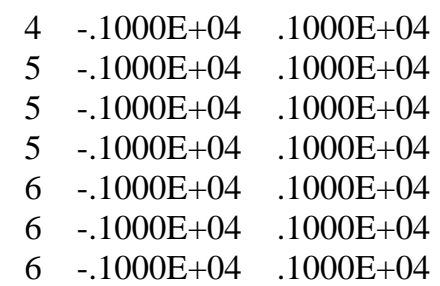

Informacoes sobre as Barras

Barra No inic No final Area Mom Inercia Comprimento CosX SenY

$\begin{array}{llllllll}1 & 1 & 2 & .3000 \mathrm{E}+01 & .1000 \mathrm{E}+01 & .2000 \mathrm{E}+01 & 1.0000 & .0000\end{array}$

$\begin{array}{llllllll}2 & 2 & 3 & .3000 \mathrm{E}+01 & .1000 \mathrm{E}+01 & .2000 \mathrm{E}+01 & 1.0000 & .0000\end{array}$

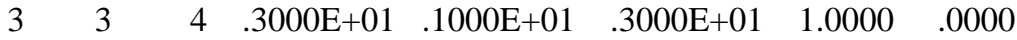

$\begin{array}{llllllll}4 & 4 & 5 & .3000 \mathrm{E}+01 & .1000 \mathrm{E}+01 & .2000 \mathrm{E}+01 & .0000 & -1.0000\end{array}$

$\begin{array}{llllllll}5 & 5 & 6 & .3000 \mathrm{E}+01 & .1000 \mathrm{E}+01 & .2000 \mathrm{E}+01 & .0000 & -1.0000\end{array}$

Cargas Aplicadas aos Nos

No Forca X Forca Y Momento Z

$3 \quad .0000 \mathrm{E}+00 \quad-.6000 \mathrm{E}+01 \quad .0000 \mathrm{E}+00$

$4 \quad .0000 \mathrm{E}+00 \quad .0000 \mathrm{E}+00 \quad-.5000 \mathrm{E}+01$

---Cargas nas Barras---

Cargas Uniformemente Distribuidas

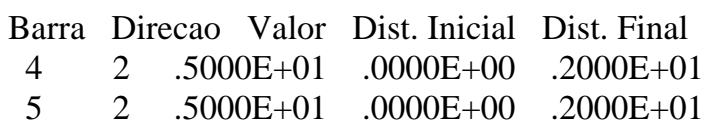

Deslocamentos Nodais

$\begin{array}{cccc}\text { No } & \text { Desl.X } & \text { Desl.Y } & \text { Rot.Z } \\ 1 & .0000 \mathrm{E}+00 & .0000 \mathrm{E}+00 & -.1130 \mathrm{E}-02 \\ 2 & .2000 \mathrm{E}-03 & -.2025 \mathrm{E}-02 & -.7785 \mathrm{E}-03 \\ 3 & .4000 \mathrm{E}-03 & -.2645 \mathrm{E}-02 & .2756 \mathrm{E}-03 \\ 4 & .7000 \mathrm{E}-03 & -.5658 \mathrm{E}-03 & .4744 \mathrm{E}-03 \\ 5 & .9205 \mathrm{E}-03 & -.2829 \mathrm{E}-03 & -.3811 \mathrm{E}-03 \\ 6 & .0000 \mathrm{E}+00 & .0000 \mathrm{E}+00 & .0000 \mathrm{E}+00\end{array}$

Reacoes nos Vinculos

$$
\begin{array}{cccc}
\text { No } & \text { Forca X } & \text { Forca Y } & \multicolumn{2}{c}{\text { Momento Z }} \\
1 & -.3000 \mathrm{E}+01 & -.1757 \mathrm{E}+01 & -.2665 \mathrm{E}-14 \\
6 & -.1309 \mathrm{E}+02 & .4243 \mathrm{E}+01 & .1166 \mathrm{E}+02
\end{array}
$$

Esforcos nas Extremidades das barras

$$
\text { Inicio da Barra Fim da Barra }
$$

$$
\begin{array}{cccccccc}
\text { Barras } & \text { FX } & \text { FY } & \text { MZ } & \text { FX } & \text { FY } & \text { MZ } & \\
\\
1 & -.3000 \mathrm{E}+01 & .1757 \mathrm{E}+01 & -.2665 \mathrm{E}-14 & .3000 \mathrm{E}+01 & -.1757 \mathrm{E}+01 & .3514 \mathrm{E}+01 \\
2 & -.3000 \mathrm{E}+01 & .1757 \mathrm{E}+01 & -.3514 \mathrm{E}+01 & .3000 \mathrm{E}+01 & -.1757 \mathrm{E}+01 & .7027 \mathrm{E}+01 \\
3 & -.3000 \mathrm{E}+01 & -.4243 \mathrm{E}+01 & -.7027 \mathrm{E}+01 & .3000 \mathrm{E}+01 & .4243 \mathrm{E}+01 & -.5702 \mathrm{E}+01 \\
4 & .4243 \mathrm{E}+01 & -.6909 \mathrm{E}+01 & .7022 \mathrm{E}+00 & -.4243 \mathrm{E}+01 & -.3091 \mathrm{E}+01 & -.4519 \mathrm{E}+01 \\
5 & .4243 \mathrm{E}+01 & .3091 \mathrm{E}+01 & .4519 \mathrm{E}+01 & -.4243 \mathrm{E}+01 & -.1309 \mathrm{E}+02 & .1166 \mathrm{E}+02
\end{array}
$$




\section{Arquivos de entrada e saída para o exemplo 3:}

\section{Arquivo de Entrada de Dados:}

NOME DA TRELICA ESPACIAL:

'torre'

NUMERO DE NOS, BARRAS E TIPOS DE MATERIAIS:

$9,20,2$

METODO UTILIZADO: 1=GRAD, 2=NEWTON, 3=Q-NEWTON, 4=GASEI

'2'

BUSCA UTILIZADA: N=APROX, S=EXATA

'S'

VALOR DA RELAXACAO

1

CARACTERISTICAS DO MATERIAL: TIPO,AREA,MODULO DE ELASTICIDADE

$1,35,2.1 \mathrm{E}+4$

$2,7.5,2.1 \mathrm{E}+4$

NUMERO DO NO E COORDENADAS X,Y E Z DOS NOS:

$1,0,0,0$

$2,400,0,0$

$3,0,400,0$

$4,400,400,0$

$5,100,100,1000$

$6,300,100,1000$

$7,100,300,1000$

$8,300,300,1000$

$9,200,200,2000$

NUMERO DE NOS COM VINCULOS RIGIDOS:

4

NÚMERO DO NO E CONDICAO DE VINCULACAO X,Y,Z:

$1,1,1,1$

$2,1,1,1$

$3,1,1,1$

$4,1,1,1$

NUMERO DE DESLOCAMENTOS COM RESTRICAO

3

NUMERO DO DESLOCAMENTO, LIM. INFERIOR E LIM. SUPERIOR

$13,-4,50$

$19,-4,50$

$25,-100,20$

NUMERO DA BARRA, NUMERO DO NO INICIAL E FINAL, TIPO DE MATERIAL:

$1,1,5,1$

$2,2,6,1$

$3,3,7,1$

$4,4,8,1$

$5,5,9,1$

$6,6,9,1$

$7,7,9,1$

$8,8,9,1$

$9,1,6,2$

$10,2,5,2$

$11,3,8,2$

$12,4,7,2$

$13,1,7,2$

$14,3,5,2$

$15,2,8,2$

$16,4,6,2$

$17,5,6,2$

$18,7,8,2$

$19,5,7,2$ 
$20,6,8,2$

NUMERO DE NOS CARREGADOS:

3

NUMERO DO NO E CARREGAMENTO SEGUNDO X,Y,Z:

$6,-300,0,0$

$8,-300,0,0$

$9,400,0,-3000$

\section{Arquivo de Saída de Resultados:}

PROGRAMA PARA TRELIÇA ESPACIAL

TRELICA: 'torre'

NUMERO DE NOS $=9$

NUMERO DE BARRAS $=20$

MUMERO DE TIPOS DE MATERIAIS $=2$

TIPO DO MATERIAL AREA MODULO DE ELASTICIDADE

$1 \quad 35.0000 \quad 21000.000$

$2 \quad 7.5000 \quad 21000.000$

A minimizacao foi feita pelo Metodo de Newton

A minimizacao utilizou a busca unidimens. exata

\begin{tabular}{|c|c|c|c|}
\hline \multicolumn{4}{|c|}{ INFORMACOES SOBRE OS NOS } \\
\hline NO & COORD & \multicolumn{2}{|c|}{ COORD Y } \\
\hline 1 & .00 & .00 & .00 \\
\hline 2 & 400.00 & .00 & .00 \\
\hline 3 & .00 & 400.00 & .00 \\
\hline 4 & 400.00 & 400.00 & .00 \\
\hline 5 & 100.00 & 100.00 & 1000.00 \\
\hline 6 & 300.00 & 100.00 & 1000.00 \\
\hline 7 & 100.00 & 300.00 & 1000.00 \\
\hline 8 & 300.00 & 300.00 & 1000.00 \\
\hline 9 & 200.00 & 200.00 & 2000.00 \\
\hline NO & VINC.X & VINC.Y & VINC.Z \\
\hline 1 & 1 & 1 & \\
\hline 2 & 1 & 1 & \\
\hline 3 & 1 & 1 & \\
\hline 4 & 1 & 1 & \\
\hline 5 & 0 & 0 & \\
\hline 6 & 0 & 0 & \\
\hline 7 & 0 & 0 & \\
\hline 8 & 0 & 0 & \\
\hline 9 & 0 & 0 & \\
\hline
\end{tabular}

INFORMACOES SOBRE OS NOS: REST. A DESLOC. E GIROS

NO LIM. INF. LIM. SUP.

$1-.1000 \mathrm{E}+04 \quad .1000 \mathrm{E}+04$

$1-.1000 \mathrm{E}+04 \quad .1000 \mathrm{E}+04$

$1-.1000 \mathrm{E}+04 \quad .1000 \mathrm{E}+04$

$2-.1000 \mathrm{E}+04 \quad .1000 \mathrm{E}+04$

$2-.1000 \mathrm{E}+04 \quad .1000 \mathrm{E}+04$

$2-.1000 \mathrm{E}+04 \quad .1000 \mathrm{E}+04$

$3-.1000 \mathrm{E}+04 \quad .1000 \mathrm{E}+04$

$3-.1000 \mathrm{E}+04 \quad .1000 \mathrm{E}+04$

$3-.1000 \mathrm{E}+04 \quad .1000 \mathrm{E}+04$

$4-.1000 \mathrm{E}+04 \quad .1000 \mathrm{E}+04$

$4-.1000 \mathrm{E}+04 \quad .1000 \mathrm{E}+04$

$4-.1000 \mathrm{E}+04 \quad .1000 \mathrm{E}+04$

$5-.4000 \mathrm{E}+01 \quad .5000 \mathrm{E}+02$ 


$\begin{array}{lll}5 & -.1000 \mathrm{E}+04 & .1000 \mathrm{E}+04 \\ 5 & -.1000 \mathrm{E}+04 & .1000 \mathrm{E}+04 \\ 6 & -.1000 \mathrm{E}+04 & .1000 \mathrm{E}+04 \\ 6 & -.1000 \mathrm{E}+04 & .1000 \mathrm{E}+04 \\ 6 & -.1000 \mathrm{E}+04 & .1000 \mathrm{E}+04 \\ 7 & -.4000 \mathrm{E}+01 & .5000 \mathrm{E}+02 \\ 7 & -.1000 \mathrm{E}+04 & .1000 \mathrm{E}+04 \\ 7 & -.1000 \mathrm{E}+04 & .1000 \mathrm{E}+04 \\ 8 & -.1000 \mathrm{E}+04 & .1000 \mathrm{E}+04 \\ 8 & -.1000 \mathrm{E}+04 & .1000 \mathrm{E}+04 \\ 8 & -.1000 \mathrm{E}+04 & .1000 \mathrm{E}+04 \\ 9 & -.1000 \mathrm{E}+03 & .2000 \mathrm{E}+02 \\ 9 & -.1000 \mathrm{E}+04 & .1000 \mathrm{E}+04 \\ 9 & -.1000 \mathrm{E}+04 & .1000 \mathrm{E}+04\end{array}$

INFORMACOES SOBRE AS BARRAS BARRA NO INIC NO FINAL

$\begin{array}{ccc}1 & 1 & 5 \\ 2 & 2 & 6 \\ 3 & 3 & 7 \\ 4 & 4 & 8 \\ 5 & 5 & 9 \\ 6 & 6 & 9 \\ 7 & 7 & 9 \\ 8 & 8 & 9 \\ 9 & 1 & 6 \\ 10 & 2 & 5 \\ 11 & 3 & 8 \\ 12 & 4 & 7 \\ 13 & 1 & 7 \\ 14 & 3 & 5 \\ 15 & 2 & 8 \\ 16 & 4 & 6 \\ 17 & 5 & 6 \\ 18 & 7 & 8 \\ 19 & 5 & 7 \\ 20 & 6 & 8\end{array}$

\begin{tabular}{ccccccc} 
BARRA & AREA & \multicolumn{1}{c}{ COMPRIMENTO } & CX & CY \\
1 & 35.0000 & 1009.950 & .0990 & .0990 & .9901 \\
2 & 35.0000 & 1009.950 & -.0990 & .0990 & .9901 \\
3 & 35.0000 & 1009.950 & .0990 & -.0990 & .9901 \\
4 & 35.0000 & 1009.950 & -.0990 & -.0990 & .9901 \\
5 & 35.0000 & 1009.950 & .0990 & .0990 & .9901 \\
6 & 35.0000 & 1009.950 & -.0990 & .0990 & .9901 \\
7 & 35.0000 & 1009.950 & .0990 & -.0990 & .9901 \\
8 & 35.0000 & 1009.950 & -.0990 & -.0990 & .9901 \\
9 & 7.5000 & 1048.809 & .2860 & .0953 & .9535 \\
10 & 7.5000 & 1048.809 & -.2860 & .0953 & .9535 \\
11 & 7.5000 & 1048.809 & .2860 & -.0953 & .9535 \\
12 & 7.5000 & 1048.809 & -.2860 & -.0953 & .9535 \\
13 & 7.5000 & 1048.809 & .0953 & .2860 & .9535 \\
14 & 7.5000 & 1048.809 & .0953 & -.2860 & .9535 \\
15 & 7.5000 & 1048.809 & -.0953 & .2860 & .9535 \\
16 & 7.5000 & 1048.809 & -.0953 & -.2860 & .9535 \\
17 & 7.5000 & 200.000 & 1.0000 & .0000 & .0000 \\
18 & 7.5000 & 200.000 & 1.0000 & .0000 & .0000 \\
19 & 7.5000 & 200.000 & .0000 & 1.0000 & .0000 \\
20 & 7.5000 & 200.000 & .0000 & 1.0000 & .0000 \\
CARGAS APLICADAS AOS NOS
\end{tabular}




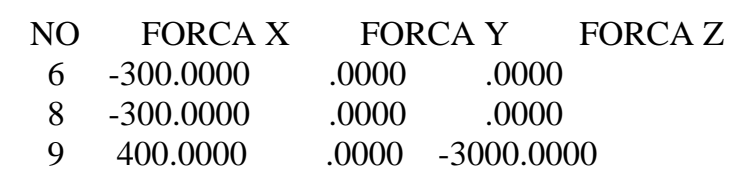

\begin{tabular}{cccc}
\multicolumn{4}{c}{ DESLOCAMENTOS NODAIS } \\
$\begin{array}{cccc}\text { NO } & \text { DESL.X } & \text { DESL.Y } & \text { DESL.Z } \\
1 & .000000 & .000000 & .000000 \\
2 & .000000 & .000000 & .000000 \\
3 & .000000 & .000000 & .000000 \\
4 & .000000 & .000000 & .000000 \\
5 & -4.000000 & -.000580 & .383107 \\
6 & -4.109773 & -.041250 & -1.613102 \\
7 & -4.000000 & .000580 & .383107 \\
8 & -4.109773 & .041250 & -1.613102 \\
9 & 17.256459 & .000000 & -1.459848\end{array}$
\end{tabular}

\begin{tabular}{|c|c|c|c|}
\hline \multicolumn{4}{|c|}{ REACOES NOS VINCULOS } \\
\hline NO & FORCA X & FORCA Y & FORCA Z \\
\hline 1 & 118.1676 & 40.8042 & 403.4766 \\
\hline 2 & -37.4722 & 113.1799 & 806.9532 \\
\hline 3 & 118.1676 & -40.8042 & 403.4766 \\
\hline 4 & -37.4722 & -113.1799 & 806.9532 \\
\hline
\end{tabular}

\section{ESFORCOS NAS EXTREMIDADES DAS BARRAS INICIO DA BARRA FIM DA BARRA}

BARRA FX FX

$\begin{array}{ccc}1 & 12.2150 & -12.2150 \\ 2 & 869.2096 & -869.2096 \\ 3 & 12.2150 & -12.2150 \\ 4 & 869.2096 & -869.2096 \\ 5 & -203.7457 & 203.7457 \\ 6 & 1426.2199 & -1426.2199 \\ 7 & -203.7457 & 203.7457 \\ 8 & 1426.2199 & -1426.2199 \\ 9 & 408.0910 & -408.0910 \\ 10 & -226.6638 & 226.6638 \\ 11 & 408.0910 & -408.0910 \\ 12 & -226.6638 & 226.6638 \\ 13 & 2.3939 & -2.3939 \\ 14 & 2.3939 & -2.3939 \\ 15 & 170.3506 & -170.3506 \\ 16 & 170.3506 & -170.3506 \\ 17 & 86.4462 & -86.4462 \\ 18 & 86.4462 & -86.4462 \\ 19 & -.9130 & .9130 \\ 20 & -64.9692 & 64.9692\end{array}$

\section{Arquivos de entrada e saída para o exemplo 4:}

\section{Arquivo de Entrada de Dados:}

NOME DA TRELICA PLANA:

'EXEMPLO 04'

NUMERO DE NOS, BARRAS E TIPOS DE MATERIAIS:

$22,41,2$

NUMERO DE PASSOS DE CARGA E DE ITERACOES MAXIMAS

50,10000

ERROS MAXIMOS EM ESFORCOS E DESLOCAMENTOS

$0.0000001,0.0000001$ 
METODO UTILIZADO: 1=GRAD, 2=NEWTON, 3=Q-NEWTON, 4=GASEI

'2'

BUSCA UTILIZADA: N=APROX, S=EXATA

'S'

VALOR DA RELAXACAO

1

CARACTERISTICAS DO MATERIAL: TIPO,AREA,MODULO DE ELASTICIDADE

$1,3.77 \mathrm{E}-4,2.1 \mathrm{E}+7$

$2,4.88 \mathrm{E}-4,2.1 \mathrm{E}+7$

NUMERO DO NO E COORDENADAS X,Y DOS NOS:

$1,0,0$

$2,2,0$

$3,4,0$

$4,6,0$

$5,8,0$

$6,10,0$

$7,12,0$

$8,14,0$

$9,16,0$

$10,18,0$

$11,20,0$

$12,0,1.5$

$13,2,1.5$

$14,4,1.5$

$15,6,1.5$

$16,8,1.5$

$17,10,1.5$

$18,12,1.5$

$19,14,1.5$

$20,16,1.5$

$21,18,1.5$

$22,20,1.5$

NUMERO DE NOS COM VINCULOS RIGIDOS:

NÚMERO DO NO E CONDICAO DE VINCULACAO X,Y:

$1,0,1$

$11,0,1$

$17,1,0$

NUMERO DE DESLOCAMENTOS COM RESTRICAO

9

NUMERO DO DESLOCAMENTO, LIM. INFERIOR E LIM. SUPERIOR

$4,-.3,1$

$6,-.3,1$

$8,-.3,1$

$10,-.3,1$

$12,-.3,1$

$14,-.3,1$

$16,-.3,1$

$18,-.3,1$

$20,-.3,1$

NUMERO DA BARRA, NUMERO DO NO INICIAL E FINAL, TIPO DE MATERIAL:

$1,1,2,2$

$2,2,3,2$

$3,3,4,2$

$4,4,5,2$

$5,5,6,2$

$6,6,7,2$

$7,7,8,2$

$8,8,9,2$

$9,9,10,2$ 
$10,10,11,2$

$11,1,12,1$

$12,2,12,1$

$13,2,13,1$

$14,3,13,1$

$15,3,14,1$

$16,4,14,1$

$17,4,15,1$

$18,5,15,1$

$19,5,16,1$

$20,6,16,1$

$21,6,17,1$

$22,6,18,1$

$23,7,18,1$

$24,7,19,1$

$25,8,19,1$

$26,8,20,1$

$27,9,20,1$

$28,9,21,1$

$29,10,21,1$

$30,10,22,1$

$31,11,22,1$

$32,12,13,2$

$33,13,14,2$

$34,14,15,2$

$35,15,16,2$

$36,16,17,2$

$37,17,18,2$

$38,18,19,2$

$39,19,20,2$

$40,20,21,2$

41,21,22,2

NUMERO DE NOS CARREGADOS:

2

NUMERO DO NO E CARREGAMENTO SEGUNDO X,Y:

$15,0,-2.2$

$19,0,-2.2$

Arquivo de Saída de Dados para apenas o último passo de carga:

\begin{tabular}{ccc}
$* * * * *$ & PASSO DE CARGA: \\
****** & ITERAÇÃO: & 4 \\
\multicolumn{4}{c}{} \\
CARGAS APLICADAS AOS NOS \\
NO & FORCA X & FORCA \\
15 & .0000 & -2.2000 \\
19 & .0000 & -2.2000 \\
\multicolumn{3}{c}{} \\
DESLOCAMENTOS NODAIS \\
NO & DESL.X & DESL.Y \\
1 & -.023832 & .000000 \\
2 & -.026406 & -.103726 \\
3 & -.025034 & -.198141 \\
4 & -.019280 & -.273585 \\
5 & -.008456 & -.300000 \\
6 & .000000 & -.300000 \\
7 & .008456 & -.300000 \\
8 & .019280 & -.273585 \\
9 & .025034 & -.198141 \\
10 & .026406 & -.103726
\end{tabular}




$\begin{array}{lll}11 & .023832 & .000000 \\ 12 & .043133 & -.004044 \\ 13 & .036939 & -.107630 \\ 14 & .027564 & -.201680 \\ 15 & .015019 & -.276783 \\ 16 & .006313 & -.298641 \\ 17 & .000000 & -.300008 \\ 18 & -.006313 & -.298641 \\ 19 & -.015019 & -.276783 \\ 20 & -.027564 & -.201680 \\ 21 & -.036939 & -.107630 \\ 22 & -.043133 & -.004044\end{array}$

\section{REACOES NOS VINCULOS}

\section{NO FORCA X FORCA Y}

$\begin{array}{lll}1 & .0000 \quad 1.3358\end{array}$

$2 \quad .0000 \quad .0000$

$3 \quad .0000 \quad .0000$

$\begin{array}{lll}4 & .0000 \quad .0000\end{array}$

$\begin{array}{lll}5 & .0000 \quad .0621\end{array}$

$\begin{array}{lll}6 & .0000 \quad 1.6042\end{array}$

$\begin{array}{lll}7 & .0000 \quad .0621\end{array}$

$\begin{array}{lll}8 & .0000 \quad .0000\end{array}$

$\begin{array}{lll}9 & .0000 \quad .0000\end{array}$

$\begin{array}{lll}10 & .0000 \quad .0000\end{array}$

$\begin{array}{lll}11 & .0000 \quad 1.3358\end{array}$

$\begin{array}{lll}12 & .0000 \quad .0000\end{array}$

$\begin{array}{lll}13 & .0000 \quad .0000\end{array}$

$14 \quad .0000 \quad .0000$

$\begin{array}{lll}15 & .0000 \quad .0000\end{array}$

$\begin{array}{lll}16 & .0000 \quad .0000\end{array}$

$\begin{array}{lll}17 & .0000 \quad .0000\end{array}$

$\begin{array}{lll}18 & .0000 \quad .0000\end{array}$

$19 \quad .0000 \quad .0000$

$\begin{array}{lll}20 & .0000 \quad .0000\end{array}$

$21 \quad .0000 \quad .0000$

$22 \quad .0000 \quad .0000$

\section{FORÇA NORMAL NAS EXTREMIDADES DAS BARRAS}

\section{INICIO DA BARRA FIM DA BARRA}

$\begin{array}{ccc}\text { BARRA } & \text { FX } & \text { FX } \\ 1 & -.0597 & .0597 \\ 2 & -1.8363 & 1.8363 \\ 3 & -3.6116 & 3.6116 \\ 4 & -5.3876 & 5.3876 \\ 5 & -4.2272 & 4.2272 \\ 6 & -4.2272 & 4.2272 \\ 7 & -5.3876 & 5.3876 \\ 8 & -3.6116 & 3.6116 \\ 9 & -1.8363 & 1.8363 \\ 10 & -.0597 & .0597 \\ 11 & 1.3327 & -1.3327 \\ 12 & -2.2309 & 2.2309 \\ 13 & 1.3416 & -1.3416 \\ 14 & -2.2323 & 2.2323 \\ 15 & 1.3683 & -1.3683 \\ 16 & -2.2352 & 2.2352 \\ 17 & 1.4673 & -1.4673 \\ 18 & 1.4555 & -1.4555 \\ 19 & -.7556 & .7556\end{array}$




$\begin{array}{lcc}20 & 1.3297 & -1.3297 \\ 21 & .0043 & -.0043 \\ 22 & 1.3297 & -1.3297 \\ 23 & -.7556 & .7556 \\ 24 & 1.4555 & -1.4555 \\ 25 & 1.4673 & -1.4673 \\ 26 & -2.2352 & 2.2352 \\ 27 & 1.3683 & -1.3683 \\ 28 & -2.2323 & 2.2323 \\ 29 & 1.3416 & -1.3416 \\ 30 & -2.2309 & 2.2309 \\ 31 & 1.3327 & -1.3327 \\ 32 & 1.7795 & -1.7795 \\ 33 & 3.5635 & -3.5635 \\ 34 & 5.3539 & -5.3539 \\ 35 & 4.2346 & -4.2346 \\ 36 & 3.1650 & -3.1650 \\ 37 & 3.1650 & -3.1650 \\ 38 & 4.2346 & -4.2346 \\ 39 & 5.3539 & -5.3539 \\ 40 & 3.5635 & -3.5635 \\ 41 & 1.7795 & -1.7795\end{array}$

FORÇA CORTANTE NAS EXTREMIDADES DAS BARRAS

INICIO DA BARRA FIM DA BARRA

\begin{tabular}{|c|c|c|}
\hline BARRA & FY & FY \\
\hline 1 & .0031 & -.0031 \\
\hline 2 & .0866 & -.0866 \\
\hline 3 & .1358 & -.1358 \\
\hline 4 & .0708 & -.0708 \\
\hline 5 & .0000 & .0000 \\
\hline 6 & .0000 & .0000 \\
\hline 7 & -.0708 & .0708 \\
\hline 8 & -.1358 & .1358 \\
\hline 9 & -.0866 & .0866 \\
\hline 10 & -.0031 & .0031 \\
\hline 11 & -.0597 & .0597 \\
\hline 12 & .1082 & -.1082 \\
\hline 13 & -.0568 & .0568 \\
\hline 14 & .0977 & -.0977 \\
\hline 15 & -.0481 & .0481 \\
\hline 16 & .0764 & -.0764 \\
\hline 17 & -.0336 & .0336 \\
\hline 18 & -.0191 & .0191 \\
\hline 19 & .0074 & -.0074 \\
\hline 20 & -.0026 & .0026 \\
\hline 21 & .0000 & .0000 \\
\hline 22 & .0026 & -.0026 \\
\hline 23 & -.0074 & .0074 \\
\hline 24 & .0191 & -.0191 \\
\hline 25 & .0336 & -.033 \\
\hline 26 & -.0764 & .0764 \\
\hline 27 & .0481 & -.048 \\
\hline 28 & -.0977 & $.097^{\prime}$ \\
\hline 29 & .0568 & -.0568 \\
\hline 30 & -.1082 & .108 \\
\hline 31 & .0597 & -.0597 \\
\hline 32 & -.0924 & .092 \\
\hline 33 & -.1684 & .1684 \\
\hline 34 & -.2023 & .2023 \\
\hline
\end{tabular}




$\begin{array}{lrr}35 & -.0465 & .0465 \\ 36 & -.0022 & .0022 \\ 37 & .0022 & -.0022 \\ 38 & .0465 & -.0465 \\ 39 & .2023 & -.2023 \\ 40 & .1684 & -.1684 \\ 41 & .0924 & -.0924\end{array}$

ERRO EM FORÇAS $=4.350146268533275 \mathrm{E}-011$

ERRO EM DESLOCAMENTOS $=3.313930291082568 \mathrm{E}-012$ 